\title{
Annual Dialysis Data Report 2016, JSDT Renal Data Registry
}

\author{
Ikuto Masakane ${ }^{1,2^{*}}$, Masatomo Taniguchi ${ }^{1}$, Shigeru Nakai ${ }^{1}$, Kenji Tsuchida', Atsushi Wada', Satoshi Ogata', \\ Takeshi Hasegawa', Takayuki Hamano ${ }^{1}$, Norio Hanafusa', Junichi Hoshino', Shunsuke Goto ${ }^{1}$, Keiichi Yamamoto', \\ Jun Minakuchi ${ }^{1}$, Hidetomo Nakamoto ${ }^{1}$ and on behalf of Japanese Society for Dialysis Therapy Renal Data Registry \\ Committee
}

\begin{abstract}
The annual survey of The Japanese Society for Dialysis Therapy Renal Data Registry (JRDR) was conducted for 4396 dialysis facilities at the end of 2016, among which 4336 facilities (98.6\%) responded. The response rate of the 2016 survey was comparable with the past, even though it was the second year after the new anonymization method. The number of chronic dialysis patients in Japan continues to increase every year; it has reached 329,609 at the end of 2016. The mean age was 68.15 years. The prevalence rate was 2597 patients per million population. Diabetic nephropathy was the most common primary disease among the prevalent dialysis patients (38.8\%), followed by chronic glomerulonephritis (28.8\%) and nephrosclerosis (9.9\%). The rate of diabetic nephropathy and nephrosclerosis has been increasing year by year, whereas that of chronic glomerulonephritis was declining. The number of incident dialysis patients during 2016 was 39,344; it has remained stable since 2008. The average age was 69.40 years, and diabetic nephropathy (43.2\%) was the most common cause in the incident dialysis patients. These patients caused by diabetes did not change in number for recent several years. Thirty-one thousand six hundred eight patients died in 2016; the crude mortality rate was $9.7 \%$. The patients treated by hemodiafiltration (HDF) have been increasing rapidly from the revision of medical reimbursement for HDF therapy in 2012. It has attained 76,836 patients at the end of 2016, which were 21,503 greater than that in 2015. The number of peritoneal dialysis (PD) patients was 9021 in 2016, which had been slightly decreasing since 2014; $20.3 \%$ of PD patients treated in the combination of hemodialysis (HD) or HDF therapy. Six hundred thirty-five patients were treated by home HD therapy at the end of 2016; it increased by 63 from 2015.
\end{abstract}

Trial registration: JRDR was approved by the ethical committee of JSDT and has been registered in "University hospital Medical Information Network (UMIN) Clinical Trials Registry" as an approved number of UMIN000018641 on August 8, 2015.

\section{Part I. JRDR 2016 annual data report: general remarks \\ Introduction}

The Japanese Society for Dialysis Therapy (JSDT) has been conducting a survey on the status of chronic dialysis therapy in Japan at the end of every year since 1968; this survey, known as the JSDT Renal Data Registry (JRDR), covers almost all dialysis facilities throughout

\footnotetext{
* Correspondence: imasakan.aipod@seieig.or.jp

${ }^{1}$ Japanese Society for Dialysis Therapy Renal Data Registry Committee, Japanese Society for Dialysis Therapy, Tokyo, Japan

2Department Nephrology, Honcho Yabuki Clinic, 1-6-17 Honcho, Yamagata, Yamagata 990-0043, Japan
}

the country $[1,2]$. Although participating facilities are not compensated, the nearly $100 \%$ response rate means that the JSDR is largely complete and literally unbiased representation of the status of chronic dialysis in Japan, making it rather rare in the world.

Until the 2013 JRDR results were reported, there were two types of JRDR reports: prompt (unfixed) data of collected results reported at the annual meeting of the JSDT held every June and defined (fixed) data subjected to subsequent data cleaning. The prompt (unfixed) data were used to prepare "An Overview of Regular Dialysis Treatment in Japan, the Illustrated Report," which was distributed at the annual meeting. The defined (fixed) 
data were used to produce "An Overview of Regular Dialysis Treatment in Japan, the CD-ROM Report," which was distributed at the end of the year to all member facilities of the JSDT or other facilities that participated in the JRDR. In addition, a status report for the second preceding year was prepared from the defined (fixed) data and published every January in Journal of the Japanese Society for Dialysis Therapy. The results in this report served as the society's official numbers, although the numbers in the Illustrated Report were cited far more often in various settings. In addition, in the 2010s, the increase in the number of dialysis patients has slowed; in fact, the number is predicted to begin decreasing within a few years [3]. This meant that the defined (fixed) data would sometimes indicate that the numbers of dialysis patients were still increasing even if they had decreased in the prompt (unfixed) data, which could easily cause major confusion. Thus, starting with the 2014 JRDR results, the production of the Illustrated Report from the prompt (unfixed) data was discontinued, and both the Illustrated Report and CD-ROM are now prepared from the defined (fixed) data instead [4]. The Illustrated Report is now distributed at the end of the year to each dialysis facility, with the CD-ROM included in its back cover. (For reasons relating to administrative procedures, "An Overview of Regular Dialysis Treatment in Japan, the CD-ROM Report" for 2016 was sent separately.)

In December 2014, the Ethical Guidelines for Medical and Health Research Involving Human Subjects, which was issued by the Ministry of Health, Labour and Welfare and the Ministry of Education, Culture, Sports, Science, and Technology, demanded that all academic societies strictly follow ethical considerations and protect personal information in epidemiological research [5]. The JSDT has also changed its survey methods based on these guidelines; starting with the 2015 JRDR, anonymity has been enhanced to ensure the protection of personal information. Furthermore, the JRDR was

Table 1 Summary of chronic dialysis therapy in Japan, 2016

\begin{tabular}{|c|c|c|c|c|c|c|c|}
\hline \multirow{2}{*}{\multicolumn{2}{|c|}{$\begin{array}{l}\text { Number of surveyed facilities } \\
\text { Number of responded facilities }\end{array}$}} & \multicolumn{2}{|c|}{4396 facilities } & \multicolumn{4}{|c|}{ (Increase of 16 facilities, $0.4 \%$ increase) } \\
\hline & & \multicolumn{2}{|c|}{4336 facilities } & \multicolumn{4}{|c|}{ (Increase of 15 facilities, 0.3\% increase) } \\
\hline \multirow[t]{3}{*}{ Capacity } & Number of bedside consoles & \multicolumn{2}{|c|}{135,211 units } & \multicolumn{4}{|c|}{ (Increase of 1673 units, 1.3\% increase) } \\
\hline & Capacity for simultaneous HD treatments & \multicolumn{2}{|c|}{133,500 treatments } & \multicolumn{4}{|c|}{ (Increase of 1986 patients, 1.5\% increase) } \\
\hline & Maximum capacity & \multicolumn{2}{|c|}{ 444,192 patients } & \multicolumn{4}{|c|}{ (Increase of 5801 patients, 1.3\% increase) } \\
\hline \multicolumn{2}{|l|}{ Prevalent dialysis patients } & \multicolumn{2}{|c|}{329,609 patients } & \multicolumn{4}{|c|}{ (Increase of 4623 patients, 1.4\% increase) } \\
\hline & & \multicolumn{2}{|c|}{ Outpatients } & \multicolumn{2}{|c|}{ Inpatients } & \multicolumn{2}{|l|}{ Total } \\
\hline \multirow[t]{5}{*}{ Hemodialysis } & Hemodialysis (HD) & 215,858 & $(72.1)$ & 25,827 & $(85.3)$ & 241,685 & $(73.3)$ \\
\hline & Hemodiafiltration (HDF) & 72,959 & $(24.4)$ & 3877 & $(12.8)$ & 76,836 & $(23.3)$ \\
\hline & Hemofiltration (HF) & 19 & $(0.0)$ & 7 & $(0.0)$ & 26 & $(0.0)$ \\
\hline & Blood adsorption dialysis & 1360 & $(0.5)$ & 46 & $(0.2)$ & 1406 & $(0.4)$ \\
\hline & Home hemodialysis & 633 & $(0.2)$ & 2 & $(0.0)$ & 635 & $(0.2)$ \\
\hline \multirow[t]{6}{*}{ Peritoneal dialysis (PD) } & PD only & 6722 & $(2.2)$ & 468 & $(1.5)$ & 7190 & $(2.2)$ \\
\hline & PD + HD 1/week & 1521 & $(0.5)$ & 39 & $(0.1)$ & 1560 & $(0.5)$ \\
\hline & PD + HD 2/week & 165 & $(0.1)$ & 4 & $(0.0)$ & 169 & $(0.1)$ \\
\hline & PD + HD 3/week & 33 & $(0.0)$ & 5 & $(0.0)$ & 38 & $(0.0)$ \\
\hline & $\mathrm{PD}+\mathrm{HD}$ other frequencies & 61 & $(0.0)$ & 3 & $(0.0)$ & 64 & $(0.0)$ \\
\hline & Subtotal & 8502 & $(2.8)$ & 519 & $(1.7)$ & 9021 & $(2.7)$ \\
\hline Total & & 299,331 & $(100.0)$ & 30,278 & $(100.0)$ & 329,609 & $(100.0)$ \\
\hline \multicolumn{2}{|c|}{ Per million of general population } & \multicolumn{2}{|c|}{2596.7 patients } & \multicolumn{4}{|c|}{ (Increase of 39.7 patients) } \\
\hline \multicolumn{2}{|c|}{ Patients count in the night shift } & \multicolumn{6}{|c|}{ 32,431 patients } \\
\hline \multicolumn{2}{|l|}{ Incident dialysis patients } & \multicolumn{2}{|c|}{ 39,344 patients } & \multicolumn{4}{|c|}{ (Decrease of 118 patients, 0.3\% decrease) } \\
\hline \multicolumn{2}{|c|}{ Incident hemodialysis patients (including HDF) } & \multicolumn{2}{|c|}{ 37,398 patients } & & & & \\
\hline \multicolumn{2}{|c|}{ Incident peritoneal dialysis patients } & \multicolumn{2}{|c|}{1946 patients } & & & & \\
\hline \multicolumn{2}{|l|}{ Deceased patients } & 31,790 pa & nts & (Increas & 722 pati & $2.3 \%$ incr & \\
\hline
\end{tabular}

The above data were obtained from the facility survey

$P D+H D$ patients patients treated by the combination of PD and HD, HDF, hemoadsorption, or hemofiltration (excluding those who underwent only peritoneal lavage) 


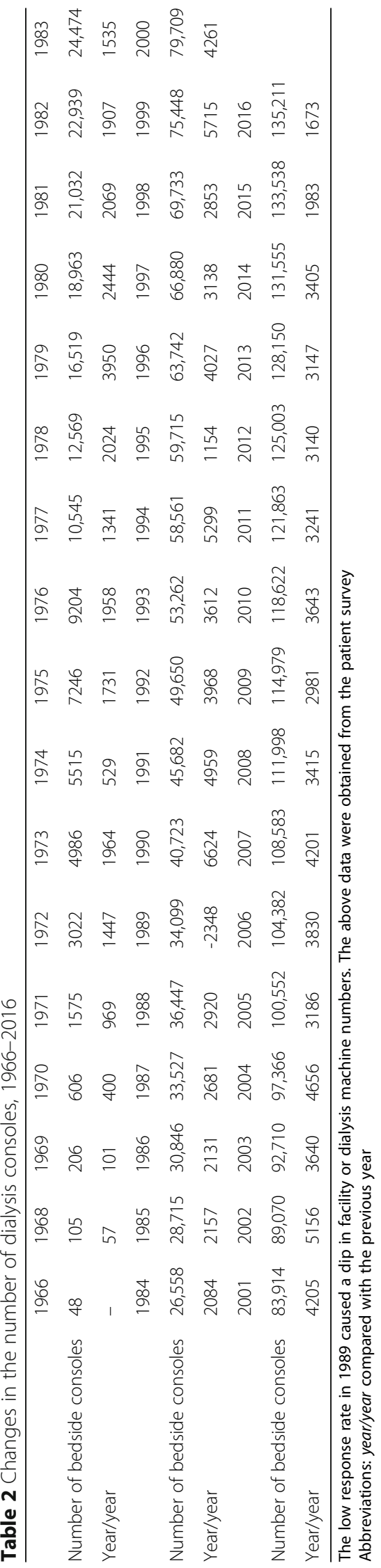




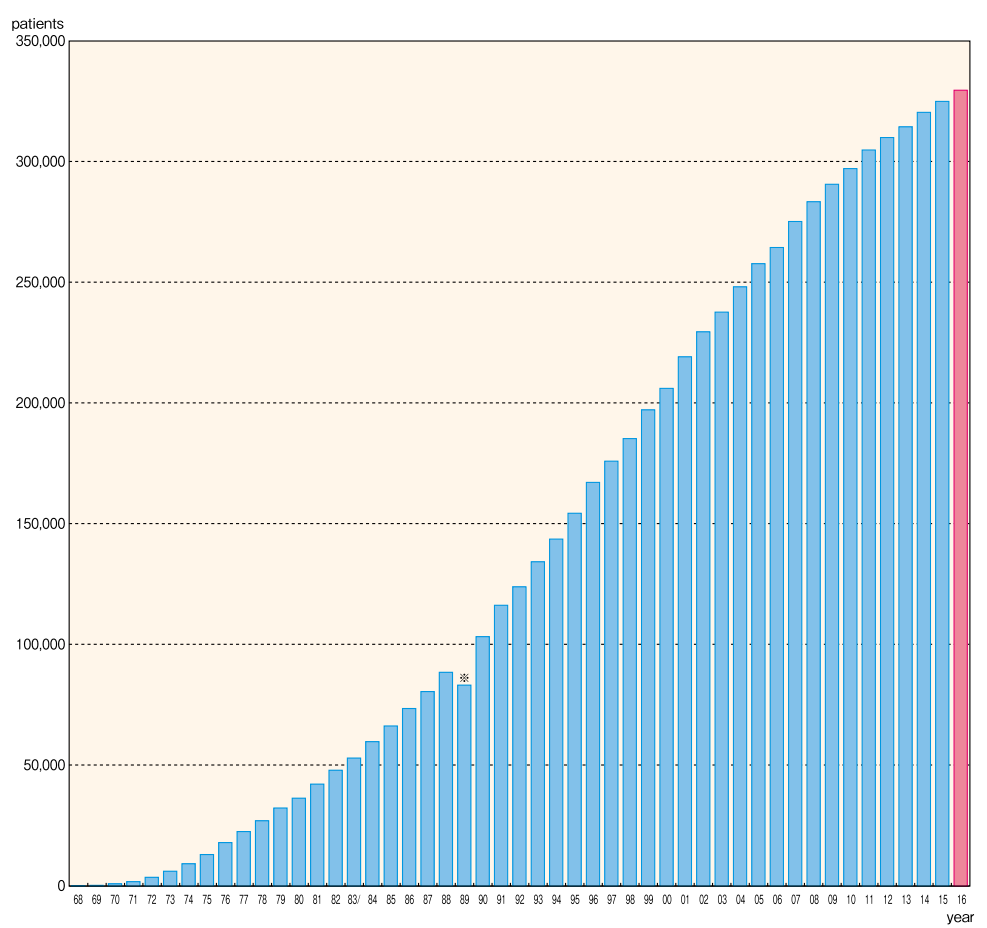

Fig. 1 Changes in the number of prevalent dialysis patients, 1968-2016. * The low response rate in 1989 caused a dip in patient numbers

reviewed in March 2015 by an ethics committee (JSDT Ethics Committee approval no. 1) to ensure ethical validity, fairness, and transparency of the survey. Following the conclusion of the abovementioned ethics review, the revised survey methods were then published in the UMIN (University hospital Medical Information Network) Clinical Trials Registry (UMIN000018641), and the review results were posted on the JSDT website [6]. Enhanced anonymization consisted of a system wherein patient information was converted to a random string of alphanumeric characters using a special algorithm, and the correspondence tables for retrieving the real names of patients were held by dialysis facilities; hence, even the JSDT main office could not retrieve patient information. The 2016 JRDR, which represented the second year of complete anonymization, involved two major issues: whether the facilities would be able to properly retrieve the anonymously distributed data and whether the secretariat would be able to properly compare the anonymized 2015 and 2016 data. Fortunately, the response rate was nearly identical to that in previous years, and we were able to publish the Illustrated Report and this Annual Report just as in previous years. We were reminded of the sincere attitude toward dialysis treatment exhibited

Table 3 Adjusted prevalent dialysis patient counts (pmp), 1983-2016

\begin{tabular}{llllllllllllllllll}
\hline & 1983 & 1984 & 1985 & 1986 & 1987 & 1988 & 1989 & 1990 & 1991 & 1992 & 1993 & 1994 & 1995 & 1996 & 1997 & 1998 & 1999 \\
$\begin{array}{l}\text { Adjusted } \\
\text { prevalent } \\
\text { dialysis }\end{array}$ & 443.7 & 497.5 & 547.8 & 604.4 & 658.8 & 721.1 & 790.0 & 835.7 & 937.6 & 995.8 & 1076.4 & 1149.4 & 1229.7 & 1328.4 & 1394.9 & 1465.2 & 1556.7 \\
patients \\
(pmp)
\end{tabular}

The low response rate in 1989 caused a dip in facility or dialysis machine numbers. The above data were obtained from the patient survey Abbreviations: $p m p$ per million population, year/year compared with the previous year 
by all those involved in it in Japan, as well as their trust in and expectations of the JSDT.

\section{Survey methods Sending and collecting surveys}

There are two types of JRDR annual surveys: facility surveys that include questions about the numbers of dialysis consoles, staff, and patients; and patient surveys that include questions about dialysis prescriptions, laboratory data, and outcome factors for individual patients at dialysis facilities. For the 2016 JRDR, USB flash drives containing facility surveys and patient surveys (the latter of which contained anonymized patient information) prepared in Excel were mailed to dialysis facilities throughout Japan in December 2016. The patient surveys included patient information that had been registered in the previous year and anonymized using the method adopted in the previous year. Each dialysis facility used a USB flash drive containing correspondence tables that had been mailed to them in 2015 in order to retrieve the patients' real names so that data such as the survival, death, or transfer of patients could be updated. Furthermore, new patients were registered, and the USB flash drive with the correspondence table was once again used to anonymize the information once entries for all patients were complete. After the anonymization, patient information in the surveys (name, sex, date of birth, etc.) was converted into random strings of alphanumeric characters of a fixed length. Each dialysis facility returned only the USB flash drive with the survey to the
JSDT main office after confirming that patients' personal information had been completely anonymized. Paperbased surveys, which had been used until 2014, were discontinued in 2015; therefore, paper-based surveys were used only for certain facilities. The initial deadline for data was January 31, 2017; however, facilities that had not responded by then were urged to participate, and the final responses were collected on June 30 . These data were incorporated into the 2016 year-end data.

\section{Survey items}

The items listed below were investigated in the 2016 JRDR. As described above, the 2016 JRDR was the second survey to incorporate enhanced anonymization. Hence, new topical survey items were not incorporated, and the contents were nearly identical to those of the 2014 and 2015 JRDR. In addition, all survey items used until 2016 are included in the members-only pages of the JSDT website (http://www.jsdt.or.jp/).

\section{Facility survey}

1. Overview and scope of facilities

- Name and contact numbers (TEL, FAX) of facility, as well as the year and month when the facility started providing dialysis treatment

- Dialysis capabilities: Capacity for simultaneous hemodialysis (HD) treatments, maximum capacity for HD treatments, and number of dialysis consoles

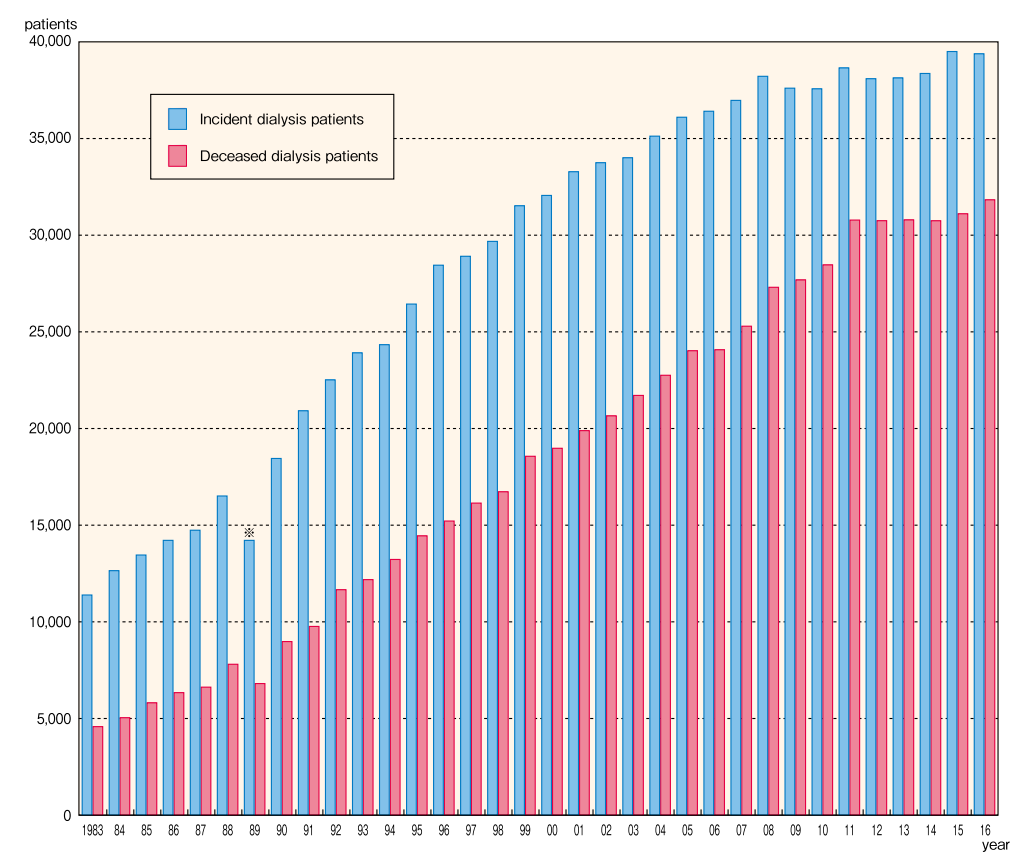

Fig. 2 Incident and deceased dialysis patient counts, 1983-2016 


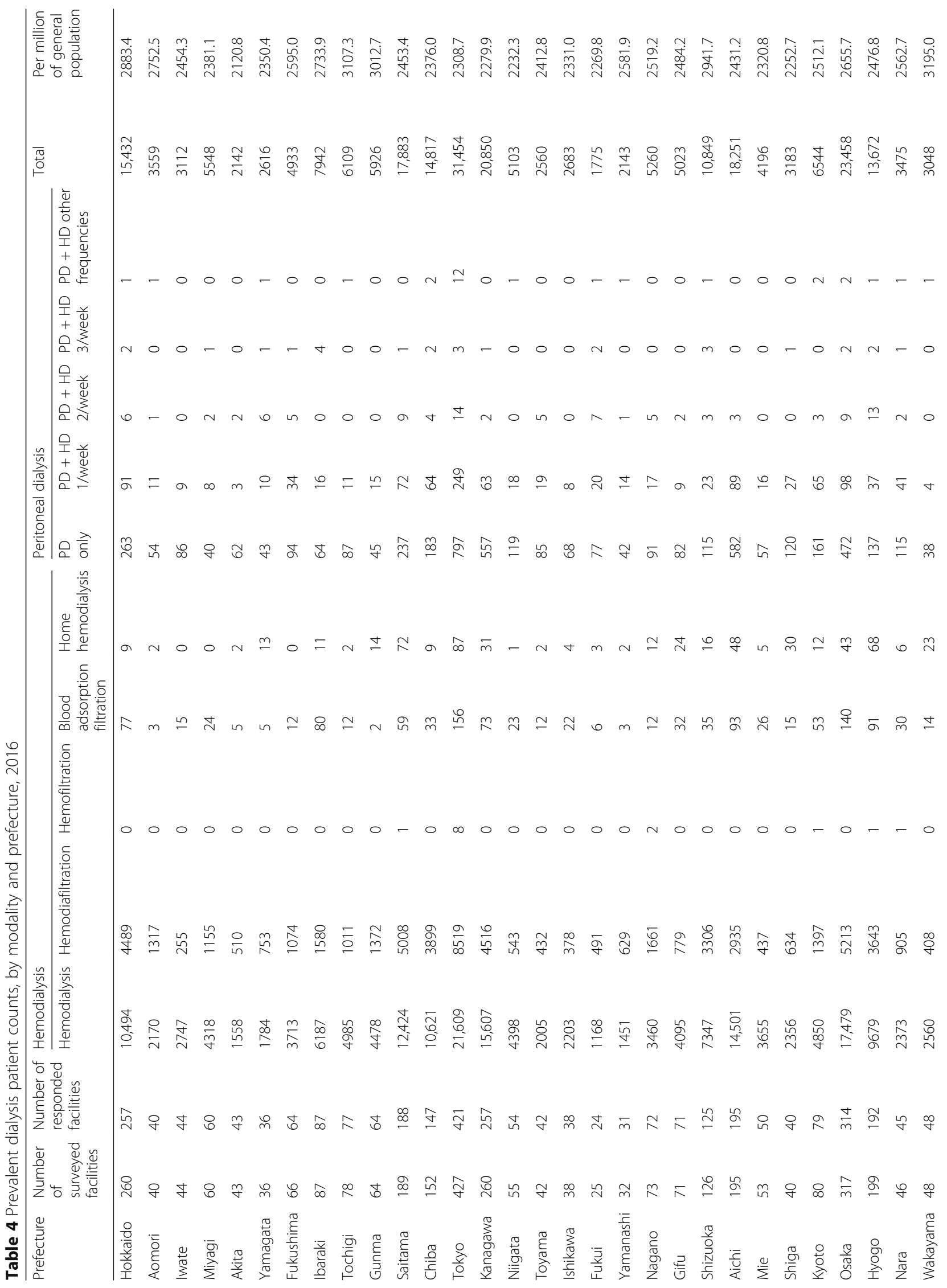




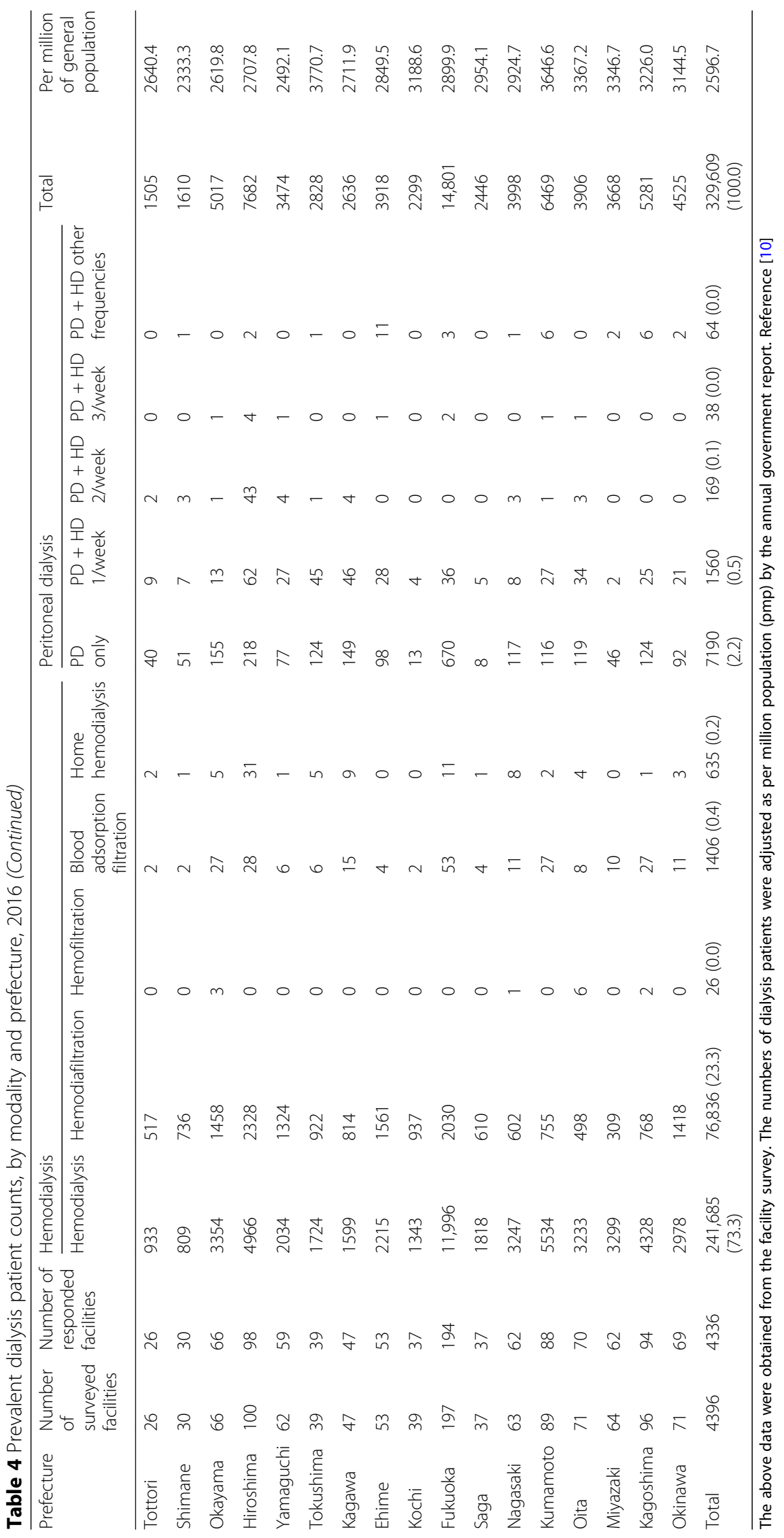


Table 5 Average age of prevalent dialysis patients, 1983-2016

\begin{tabular}{|c|c|c|c|c|c|c|c|c|c|c|c|c|c|c|c|c|c|}
\hline & 1983 & 1984 & 1985 & 1986 & 1987 & 1988 & 1989 & 1990 & 1991 & 1992 & 1993 & 1994 & 1995 & 1996 & 1997 & 1998 & 1999 \\
\hline \multirow{2}{*}{$\begin{array}{l}\text { Mean age of the prevalent dialysis } \\
\text { patients }\end{array}$} & 48.3 & 49.2 & 50.3 & 51.1 & 52.1 & 52.9 & 53.8 & 54.5 & 55.3 & 56.0 & 56.6 & 57.3 & 58.0 & 58.6 & 59.2 & 59.9 & 60.6 \\
\hline & 2000 & 2001 & 2002 & 2003 & 2004 & 2005 & 2006 & 2007 & 2008 & 2009 & 2010 & 2011 & 2012 & 2013 & 2014 & 2015 & 2016 \\
\hline $\begin{array}{l}\text { Mean age of the prevalent dialysis } \\
\text { patients }\end{array}$ & 61.2 & 61.6 & 62.2 & 62.8 & 63.3 & 63.9 & 64.4 & 64.9 & 65.3 & 65.8 & 66.2 & 66.6 & 66.9 & 67.2 & 67.5 & 67.9 & 68.2 \\
\hline
\end{tabular}

The above data were obtained from the patient survey

- Number of workers involved in dialysis treatment (e.g., doctors, nurses, clinical engineers, nutritionists, case workers)

- Number of medical dialysis specialists qualified by JSDT

2. Patient dynamics

- Number of prevalent dialysis patients at end of 2016 (number of patients by treatment modality, inpatient/outpatient)

- Number of dialysis patients undergoing nighttime dialysis in 2016

- Number of incident dialysis patients in 2016 (number of incident $\mathrm{HD}(\mathrm{F})$ and $\mathrm{PD}$ patients)

- Number of deceased patients during 2016

3. Dialysis fluid quality management

- Use of endotoxin retentive filter (ETRF)

- Dialysis fluid sampling status and sampling site of dialysis fluid during testing

- Frequency for measuring endotoxin (ET) concentration in dialysis fluid and ET concentration in dialysis fluid

- Frequency for measuring total viable microbial count (TVC) in dialysis fluid, sampling volume for TVC, cultivation medium for TVC, and TVC in dialysis fluid

\section{Patient survey}

1. Patient personal information

- Sex, date of birth, year and month of start of dialysis, year and month of transfer from another hospital, primary disease, residence (prefecture), dialysis modality, month of transfer (destination facility code), outcome category, outcome date (transfer, death, dropout, or transplantation) (destination facility code), month of death, cause of death, dates of changes, change codes, status of combined therapies involving PD with $\mathrm{HD}$ or HDF, etc., PD experience, and number of kidney transplants

2. HD/HDF therapy conditions

- Frequency of dialysis session per week, dialysis time per session, and blood flow rate

- HDF: dilution methods, substitution fluid volume per session

- Body height, pre- and post-dialysis body weight, pre-dialysis systolic blood pressure, pre-dialysis diastolic blood pressure, and pre-dialysis pulse rate

3. Laboratory findings

- Pre- and post-dialysis serum urea nitrogen (UN), pre- and post-dialysis serum creatinine concentration, pre-dialysis serum albumin concentration, pre-dialysis serum C-reactive protein (CRP) concentration, pre-dialysis serum calcium concentration, pre-dialysis serum phosphorus concentration, serum parathyroid hormone (PTH) assay method, PTH level (intact or whole PTH), pre-dialysis hemoglobin concentration, serum total cholesterol concentration (total cholesterol), and serum high-density-lipoprotein-cholesterol concentration (HDL-C)

4. Outcome factors

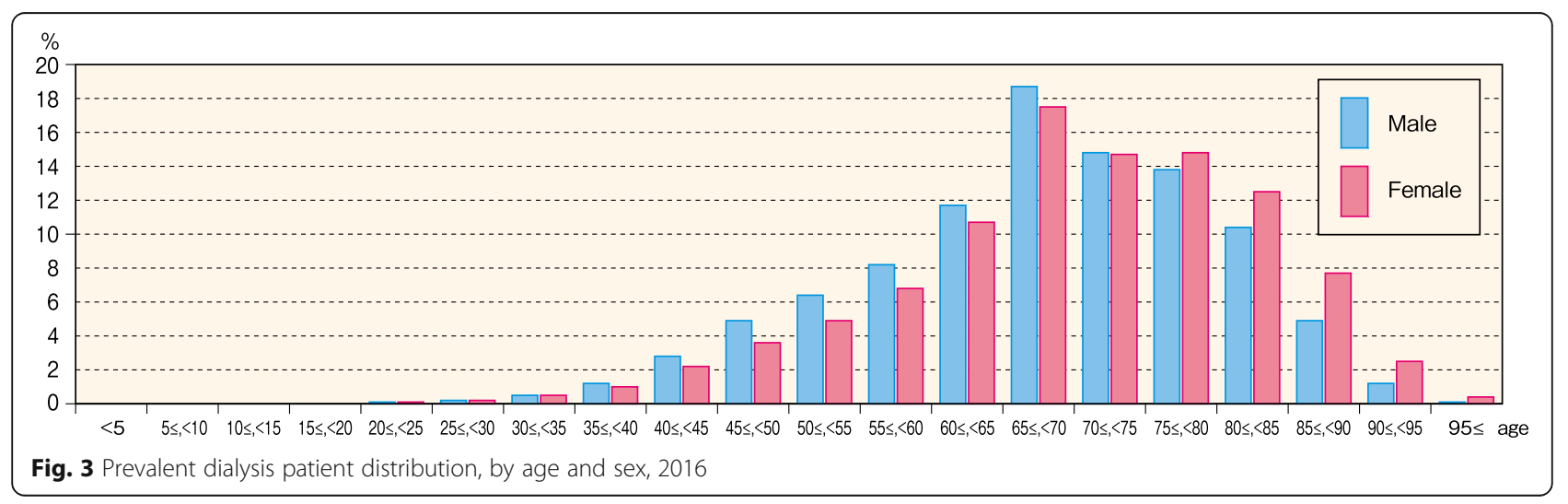




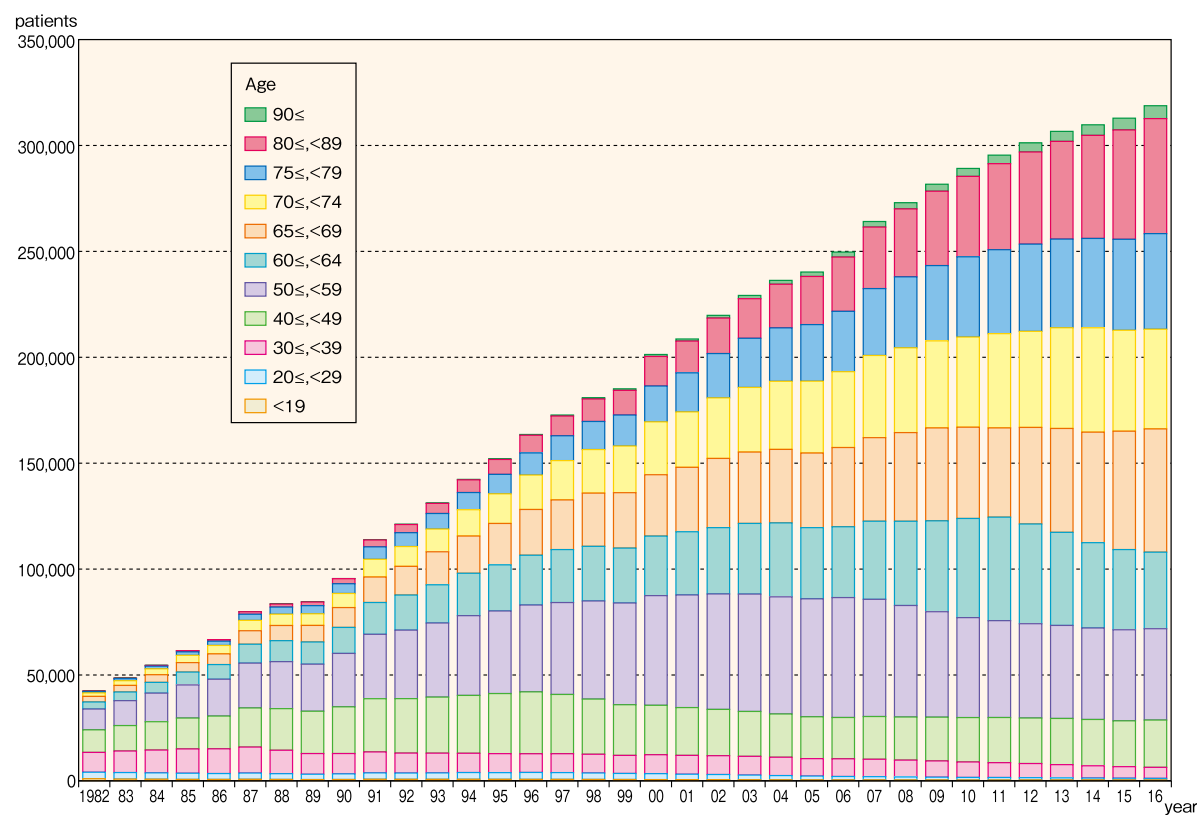

Fig. 4 Prevalent dialysis patient distribution, by age, 1982-2016

- Antihypertensive drug use, smoking, history of diabetes, history of myocardial infarction, history of cerebral hemorrhage, history of cerebral infarction, limb amputation, history of proximal femur fracture, history of encapsulating peritoneal sclerosis (EPS)

5. Peritoneal dialysis (PD) survey
- Therapeutic history: Current PD dialysis vintage, number of months in which PD was performed in 2016

- Peritoneal function: Implementation of peritoneal equilibration test (PET), 4-h creatinine concentration dialysate/plasma ratio in PET (PET Cr D/P ratio)

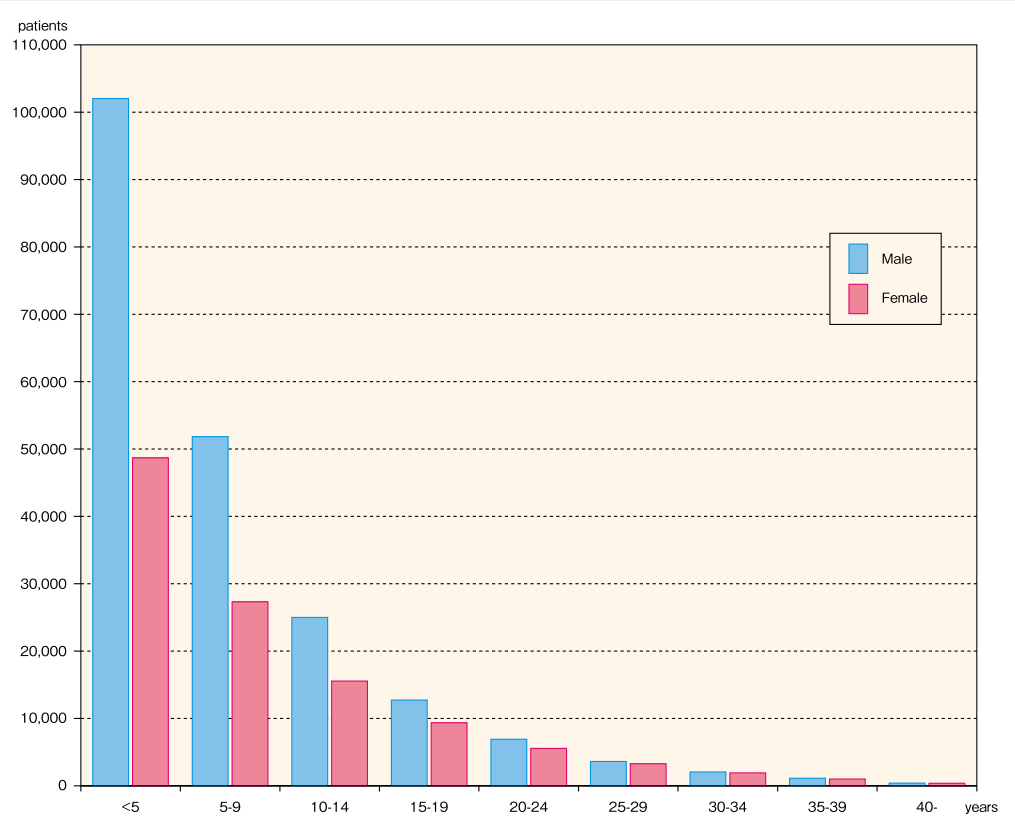

Fig. 5 Prevalent dialysis patient distribution, by dialysis vintage and sex, 2016 


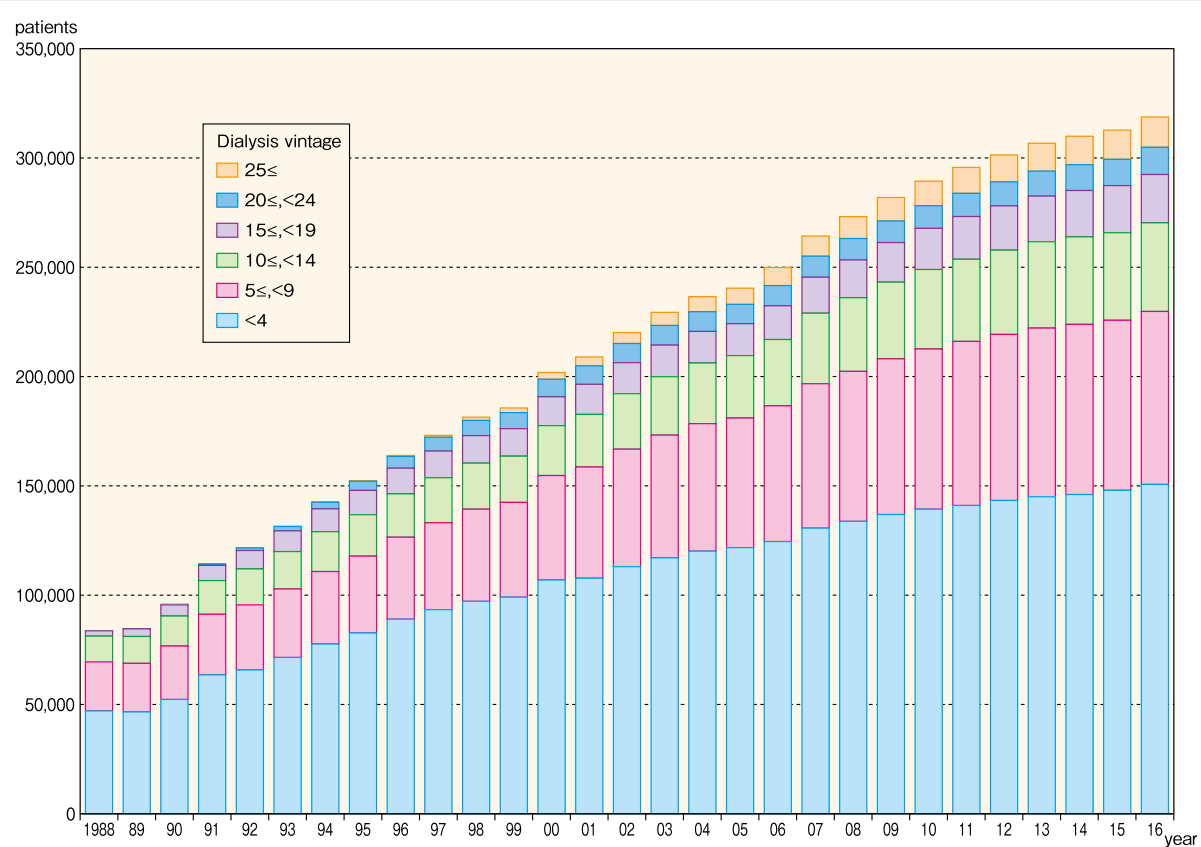

Fig. 6 Prevalent dialysis patient distribution, by dialysis vintage, 1988-2016

- Dialysis prescription: Type of PD fluid, volume of PD fluid per day, PD treatment time per day, daily urine volume, mean fluid removal volume per day, $K t / V$ by residual kidney function (residual kidney $K t / V$ ), $K t / V$ by PD (PD $K t / V$ )

- Dialysis method: Use of automated peritoneal dialysis (APD) machine, changing maneuver of PD fluid

- Infectious disease: Numbers of peritonitis during 2016 (peritonitis frequency), numbers of exit-site infections during 2016

\section{Methods for publishing JRDR results and an overview of this report}

Until the 2013 edition, the JRDR annual report was published in Japanese in every January issue of the Journal of the Japanese Society for Dialysis Therapy, and a translated version was published in Therapeutic Apheresis and Dialysis (TAD). In TAD, survey results were mostly reported in tables owing to page limitations, whereas the Illustrated Report, as per its name, provided an explanation through figures. PDFs of TAD papers and the

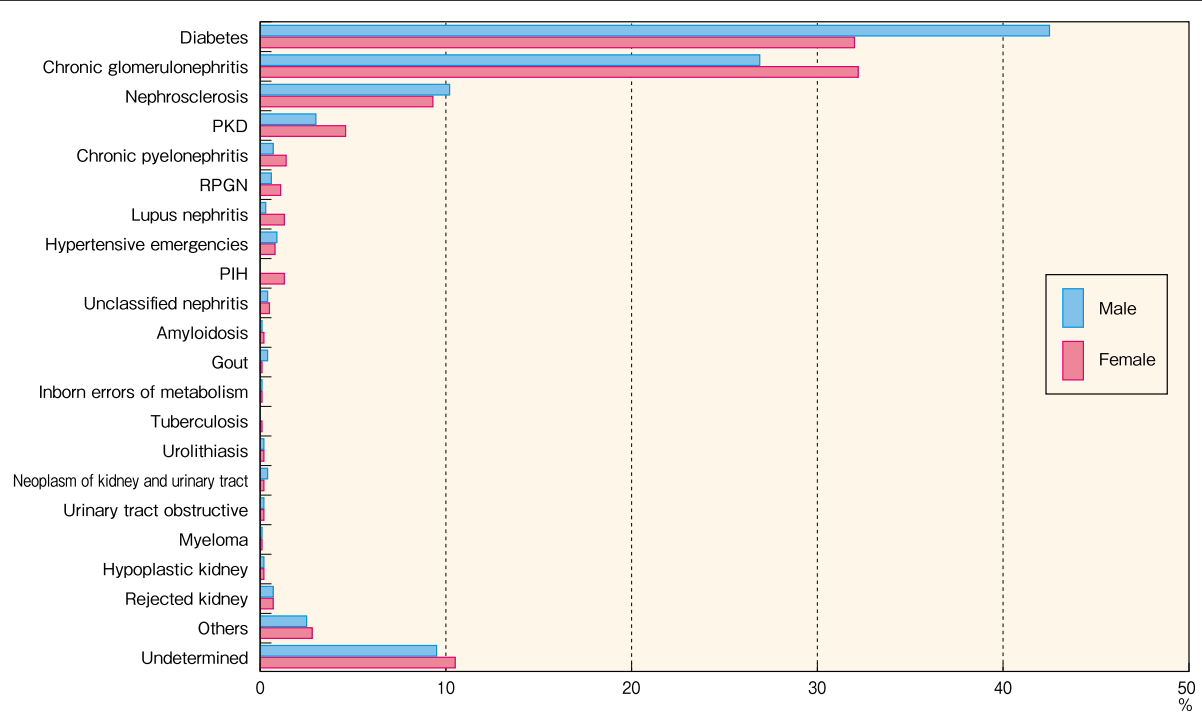

Fig. 7 Prevalent dialysis patient distribution, by primary disease and sex, 2016. Abbreviations: PIH pregnancy-induced hypertension, PKD polycystic kidney disease, RPGN rapidly progressive glomerulonephritis 


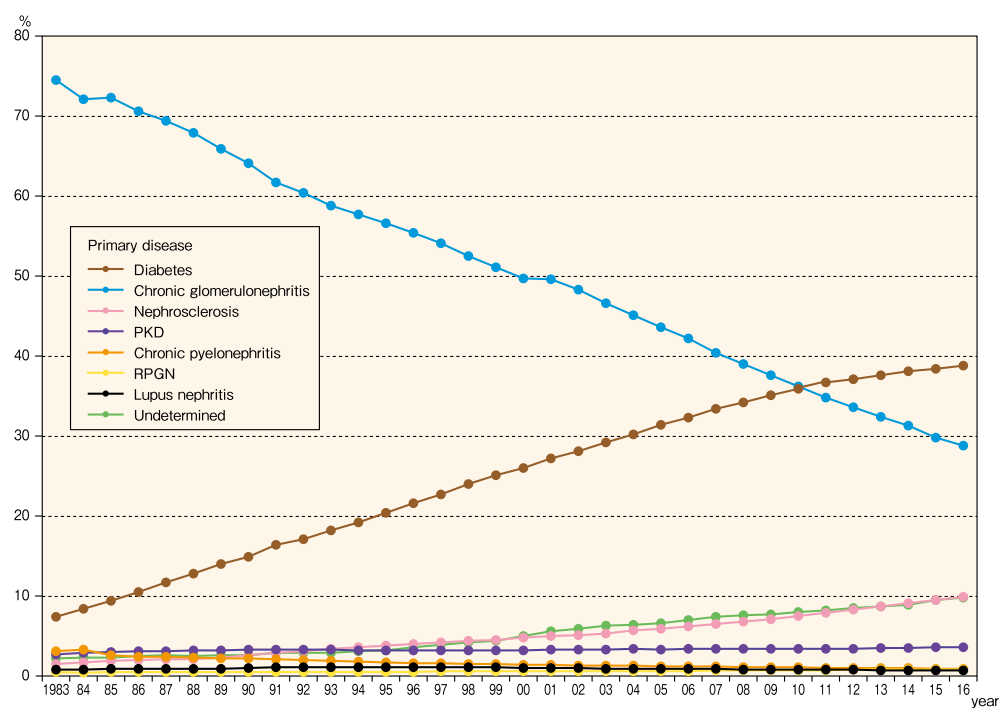

Fig. 8 Prevalent dialysis patient distribution, by primary disease, 1983-2016. Abbreviations: PKD polycystic kidney disease, RPGN rapidly progressive glomerulonephritis

Illustrated Reports, as well as their figures (in the form of PowerPoint files), can be downloaded from the JSDT website. However, the figures in the Illustrated Reports were used more often. In contrast, countries other than Japan have been requesting that the results of the JRDR be published in a form that people worldwide may easily use. Because of such circumstances, the English-language annual report has been published in Renal Replacement Therapy (RRT) since the report for 2014 [7, 8]. We expect that the PDF of the English-language annual report, an English translation of the figures as a PowerPoint file, and an Excel file of the tables, will be available to the world from the English-language website of the JSDT.
Part II. JRDR 2016 annual data report: results and discussion

Chapter 1: Basic demographics

Facility dynamics

The 2016 JRDR annual survey was conducted at $4396 \mathrm{fa}$ cilities nationwide, and responses were obtained from 4336 facilities. The number of responding facilities decreased in 2015 (by 9 facilities, or $-0.2 \%$ ) but increased again in 2016 (by 15 facilities, or $+0.3 \%$ ). Although these are strictly numbers of responses rather than response rates, there was a concern that the enhanced anonymization and accompanying discontinuation of paper-based surveys starting from the 2015 JRDR would result in a decreased response rate and the consequent underestimation

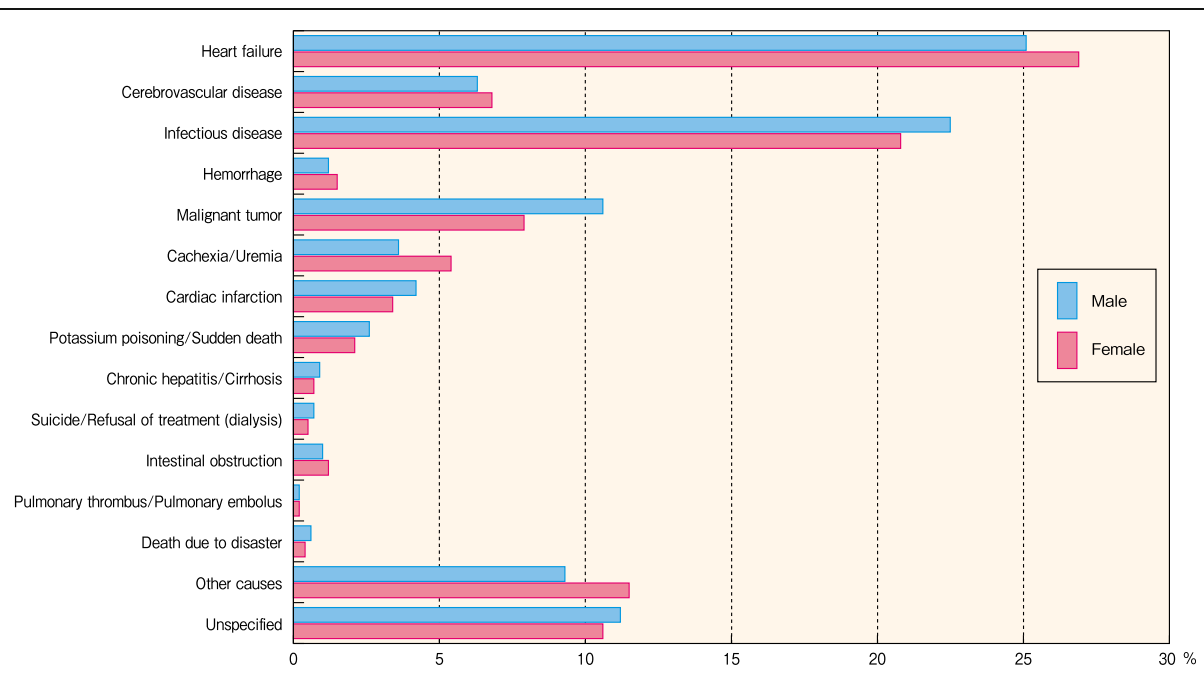

Fig. 9 Deceased dialysis patient distribution, by cause of death and sex, 2016 


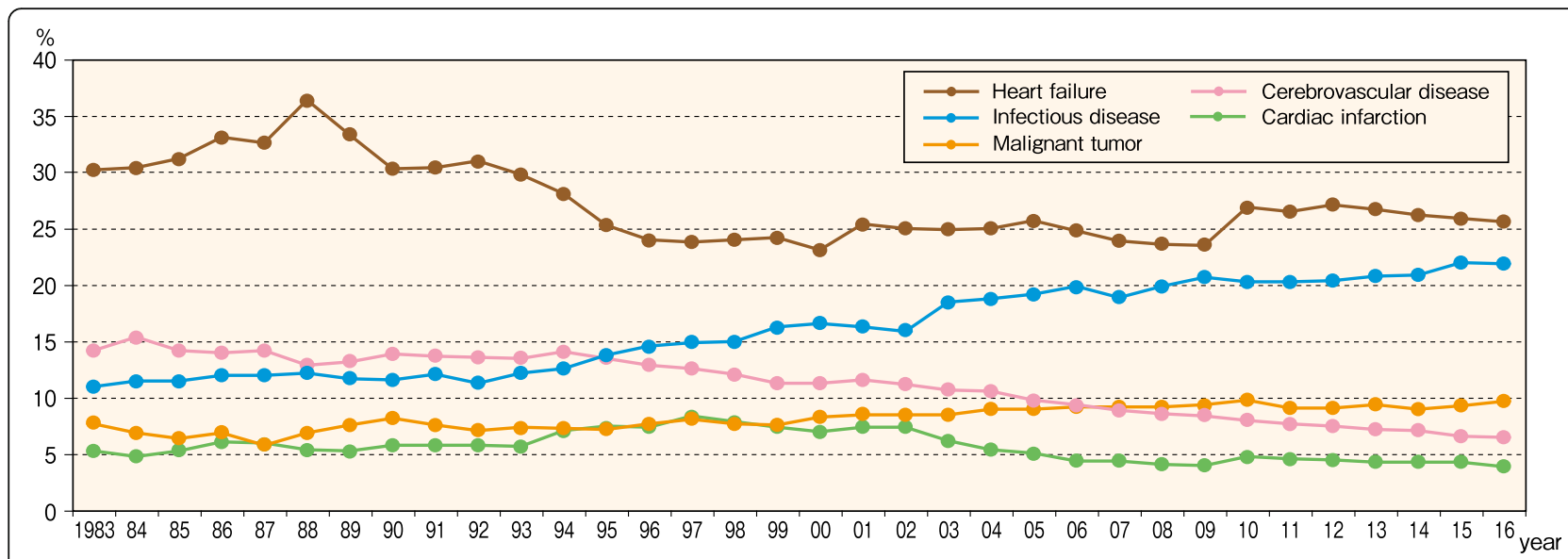

Fig. 10 Major causes of death, 1983-2016

of the number of dialysis facilities and patients. However, the response rate for facility surveys was $98.6 \%$, whereas patient surveys were able to be collected from $95.2 \%$ of all facilities; thus, the response rates were nearly identical to those from previous years. Therefore, a decrease in the number of facilities responding to the surveys does not necessarily mean a substantial decrease in the actual number of dialysis facilities. However, the increase in the number of dialysis patients has recently slowed, and future trends are therefore being noted (Table 1). The 4336 facilities that responded had 135,211 dialysis consoles (an increase of 1673 from the previous year), a simultaneous dialysis treatment capacity of 133,500 , and a maximum dialysis treatment capacity of 444,192 . Compared to the figures at the end of 2015, these figures represented increases of 1.3, 1.5, and $1.3 \%$, respectively (Tables 1 and 2).

\section{Patient dynamics}

Based on the facility surveys, a total of 329,608 patients were undergoing chronic dialysis therapy at the end of 2016; this number represents the prevalence of chronic kidney disease patients on dialysis therapy. The number of dialysis patients increased by approximately 10,000 annually through 2005, but this rate of increase has been slowing in recent years. At the end of 2014, the number of dialysis patients had increased by 6010 from the previous year; the number had increased by 4538 at the end of 2015 and by 4623 in 2016 (Fig. 1, Additional file 1: Table S1). In the figure, the decrease in the number of patients at the end of 1989 is the apparent effect of the unusually low $86 \%$ survey response rate that year [2]. In 2012, Nakai et al. [3] predicted that the number of future dialysis patients would decrease after reaching a peak of approximately 348,000 in 2021 . The number of dialysis patients per million of the Japanese population is 2596.7, an increase of 39.7 patients from the previous year; this finding means that there is 1 dialysis patient per 385.1 Japanese citizens (Table 3). The population of Japan has been decreasing since 2011; thus, the percentage of dialysis patients in the population has increased yearly. According to the United States Renal Data System, Taiwan has the highest number of dialysis patients per million population worldwide, followed by Japan [9]. Meanwhile, the count of incident dialysis patients represents the incidence of chronic kidney disease patients on dialysis therapy. Although the count of incident dialysis patients showed an upward trend until 2008, it began to decrease in 2009. Since then, faint fluctuations in the patient count have been observed, although the rate has remained largely constant. However, the count of incident dialysis patients in 2015 increased by 1135 from the previous year to 39,462 , exceeding 39,000 patients for the first time, and stood at 39,344 in 2016 (Fig. 2, Additional file 2: Table S2). In contrast, the annual count of deceased patients increased every year until 2011 but has since remained mostly constant. In 2015, the deceased patient count was 31,068 and that in 2016 was 31,790 (Fig. 2, Additional file 2: Table S2). In general,

Table 6 Annual crude death rate, 1983-2016

\begin{tabular}{|c|c|c|c|c|c|c|c|c|c|c|c|c|c|c|c|c|c|}
\hline & 1983 & 1984 & 1985 & 1986 & 1987 & 1988 & 1989 & 1990 & 1991 & 1992 & 1993 & 1994 & 1995 & 1996 & 1997 & 1998 & 199 \\
\hline \multirow[t]{2}{*}{ Crude death rate (\%) } & 9.0 & 8.9 & 9.1 & 9.0 & 8.5 & 9.2 & 7.9 & 9.6 & 8.9 & 9.7 & 9.4 & 9.5 & 9.7 & 9.4 & 9.4 & 9.2 & 9.7 \\
\hline & 2000 & 2001 & 2002 & 2003 & 2004 & 2005 & 2006 & 200 & 200 & 2009 & 20 & 20 & 2012 & 2013 & 2014 & 2015 & \\
\hline ude death rate $(\%)$ & 9.2 & 9.3 & 9.2 & 9.3 & 9.4 & 9.5 & 9.2 & 9.4 & 9.8 & 9.6 & 9.8 & 10.2 & 10.0 & 9.8 & 9.7 & 9.6 & 9.7 \\
\hline
\end{tabular}

The above data were obtained from the patient survey 


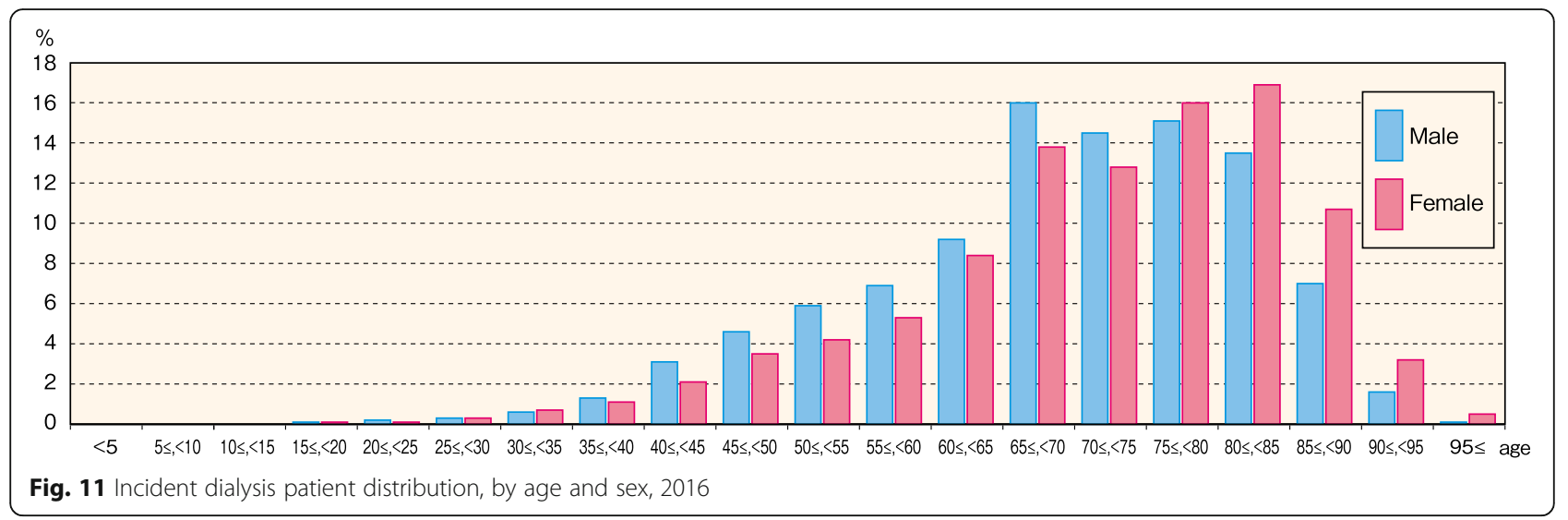

the number of patients in a given year should be equal to the number of patients in the previous year plus the incident patient count minus the deceased patient count. However, patients who discontinue dialysis because of a transplant are not counted; in addition, the deceased patient count could be underestimated, whereas the incident patient count could be overestimated. Thus, the numbers do not add up exactly.

\section{Patient count by dialysis modality and prefecture}

Starting with the 2015 JRDR, the tabulation method was changed to a method focusing on modalities such as HDF, the use of which has been increasing rapidly. The percentage of each modality was $73.3 \%$ for $\mathrm{HD}, 23.3 \%$ for HDF, $0.4 \%$ for hemofiltration (HF) and hemoadsorption dialysis, $2.7 \%$ for $\mathrm{PD}$, and $0.2 \%$ for home hemodialysis (HHD) (Table 1). The total percentage of home dialysis (PD plus HHD) in Japan is $2.9 \%$, which is the lowest in the developed world [9]. The use of HDF, particularly on-line HDF, has dramatically increased since the 2012 revision of the medical reimbursement system; the total number of patients using HDF at the end of 2016 was 76,836, an increase of 21,503 from 2015. The number of PD patients was 9021, which represented a decrease of about 300 from 9322 patients in 2015. About 20.3\% PD patients underwent combined therapy with $\mathrm{HD}$, and this percentage has remained largely constant for the past 5 years. The number of HHD patients was 635 , which was 63 patients more than that in 2015; although this represents a large rate of increase, the percentage of HHD among all modalities remains small. The number of nighttime dialysis patients was approximately 41,000-42,000 until the 2014 JRDR, but it decreased to 33,370 in 2015 and to 32,431 in 2016. This decrease may have been due to the addition of "Dialysis during hours recognized by insurance (starting after 5:00 pm or ending after 9:00 pm)" to the definition of nighttime dialysis in the 2015 JRDR. The total count of incident dialysis patients was 39,344; of these patients, 95.1\% began HD or similar modalities, whereas $4.9 \%$ began PD (a decrease from $5.6 \%$ in 2015).

Until 2014, the numbers of chronic dialysis patients by prefecture were categorized by daytime dialysis, nighttime dialysis, HHD, and PD. However, since the 2015 JRDR, the numbers of chronic dialysis patients by prefecture are now tabulated by detailed modality.

The tabulation of prefectures represents the locations of the facilities rather than those of the patients and thus does not strictly represent patient dynamics by prefecture. There are pronounced differences between regions in the number of patients per modality and per million in the population [10]. These regional differences arise from a massive number of factors that are interrelated in a complex manner; therefore, comparisons of prefectures require great care (Table 4 ).

\section{Age, sex, and dialysis vintage of prevalent patients at the end of 2016}

In the patient survey, the count of prevalent dialysis patients at the end of 2016 (specifically, the number

Table 7 Average age of incident dialysis patients, 1983-2016

\begin{tabular}{|c|c|c|c|c|c|c|c|c|c|c|c|c|c|c|c|c|c|}
\hline & 1983 & 1984 & 1985 & 1986 & 1987 & 1988 & 1989 & 1990 & 1991 & 1992 & 1993 & 1994 & 1995 & 1996 & 1997 & 1998 & 1999 \\
\hline \multirow{2}{*}{$\begin{array}{l}\text { Mean age of the incident dialysis } \\
\text { patients }\end{array}$} & 51.9 & 53.2 & 54.4 & 55.1 & 55.9 & 56.9 & 57.4 & 58.1 & 58.1 & 59.5 & 59.8 & 60.4 & 61.0 & 61.5 & 62.2 & 62.7 & 63.4 \\
\hline & 2000 & 2001 & 2002 & 2003 & 2004 & 2005 & 2006 & 2007 & 2008 & 2009 & 2010 & 2011 & 2012 & 2013 & 2014 & 2015 & 2016 \\
\hline $\begin{array}{l}\text { Mean age of the incident dialysis } \\
\text { patients }\end{array}$ & 63.8 & 64.2 & 64.7 & 65.4 & 65.8 & 66.2 & 66.4 & 66.8 & 67.2 & 67.3 & 67.8 & 67.8 & 68.4 & 68.7 & 69.0 & 69.2 & 69.4 \\
\hline
\end{tabular}

The above data were obtained from the patient survey 


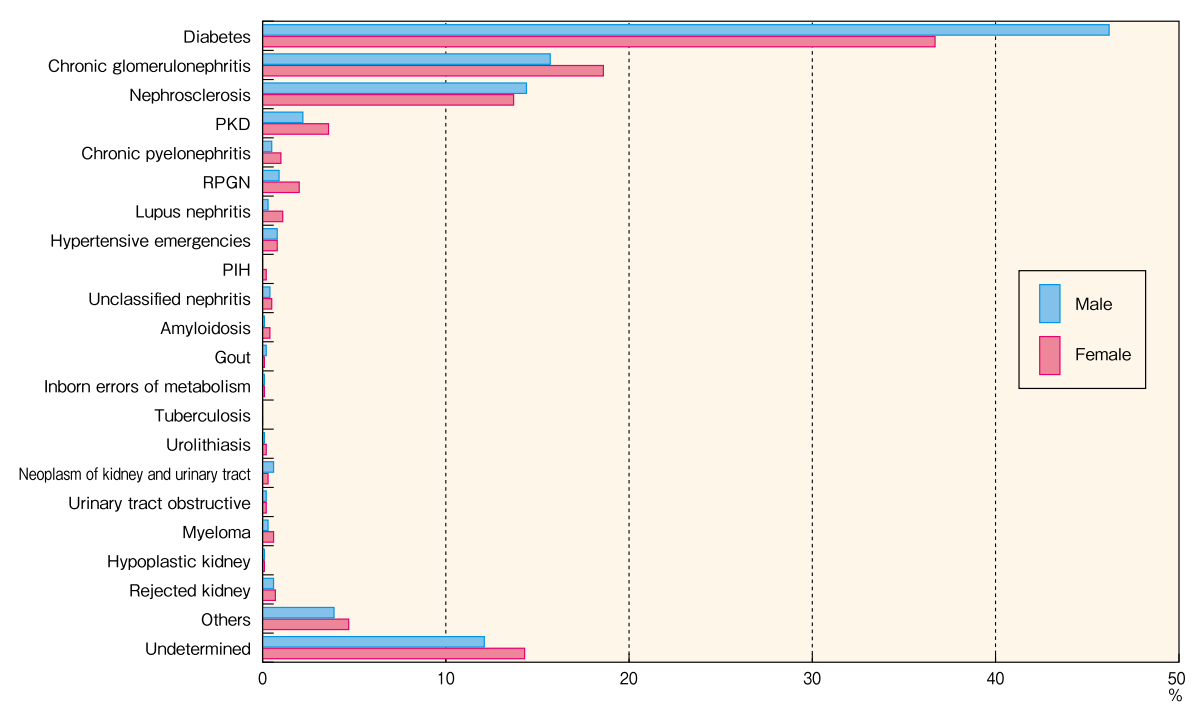

Fig. 12 Incident dialysis patient distribution, by primary disease and sex, 2016. Abbreviations: PIH pregnancy-induced hypertension, PKD polycystic kidney disease, RPGN rapidly progressive glomerulonephritis

of patients for whom age and sex data were listed) was 319,107 ; this number is 10,502 patients fewer than, or $96.8 \%$ of, the count of 329,609 from the facility surveys. The mean age of the prevalent dialysis patients was 68.15 years, which was 0.29 years higher than that in the previous year (Table 5). The mean age of men was 67.34 years, a 0.27 -year increase, whereas the mean age of women was 69.61 years, a 0.33 -year increase (Fig. 3, Additional file 3: Table S3). The most common age range for both men and women was 6569 years.
The number of patients younger than 65 years has been decreasing since 2012, reflecting an increase in age among incident patients. In other words, the increase in chronic dialysis patients in Japan is the result of an increase in patients aged 65 years and older. The percentage of old-old patients (75 years and older) also increased; there were 6095 dialysis patients aged 90 years and older (Fig. 4, Additional file 4: Table S4).

Comparisons of the dialysis vintages of prevalent patients at the end of 2016 by 5 -year segments showed that $47.3 \%$ of all patients had a dialysis vintage of $<5$ years,

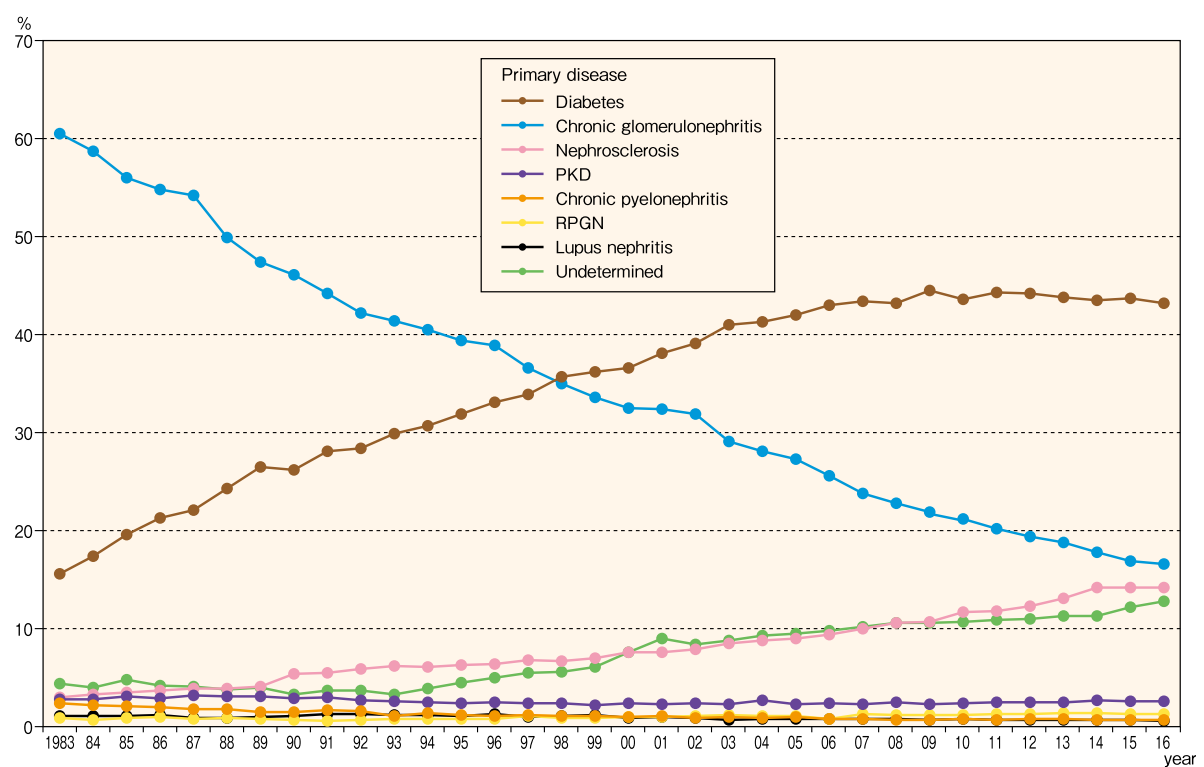

Fig. 13 Incident dialysis patient distribution, by primary disease, 1983-2016. Abbreviations: PKD polycystic kidney disease, RPGN rapidly progressive glomerulonephritis 


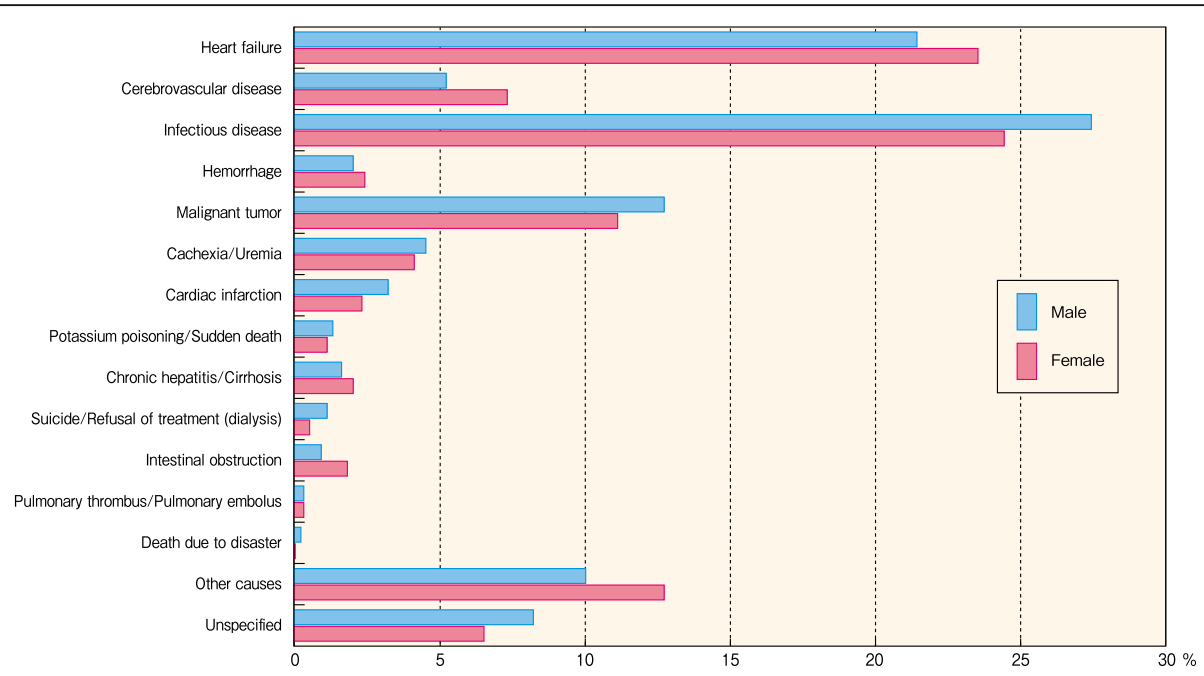

Fig. 14 Incident dialysis patient distribution, by cause of death and sex, 2016

whereas 26,313 patients had a dialysis vintage of at least 20 years, representing an increase of 922 patients $(8.3 \%$ of all patients) over the previous year. There were 793 patients $(0.2 \%$ of all patients) with a dialysis vintage of 40 years or longer (Fig. 5, Additional file 5: Table S5). The longest dialysis vintage was 48 years and 4 months.

In all dialysis vintage segments, there were more men in terms of absolute numbers; however, the difference became smaller as the dialysis vintage lengthened. Chronic dialysis therapy was first covered by insurance in Japan in 1967; the effects of this coverage and of differences in natural history are thought to result in fluctuations in the male/female ratio. The percentage of patients with vintages $<5$ years has gradually decreased every year, whereas the number of patients with long vintages has been increasing; the percentage of patients with vintages of at least 10 years has reached $27.9 \%$. Patients with vintages of 20 years or longer accounted for $<1 \%$ of all patients at the end of 1992, but reached $8.3 \%$ at the end of 2016 (Fig. 6, Additional file 6: Table S6).

\section{Primary diseases in prevalent patients at the end of 2016}

The most common primary disease in prevalent dialysis patients at the end of 2016 was diabetic nephropathy, followed by chronic glomerulonephritis and nephrosclerosis (38.8, 28.8, and 9.9\%, respectively). However, 9.8\% had an unknown primary disease (Fig. 7, Additional file 7: Table S7).

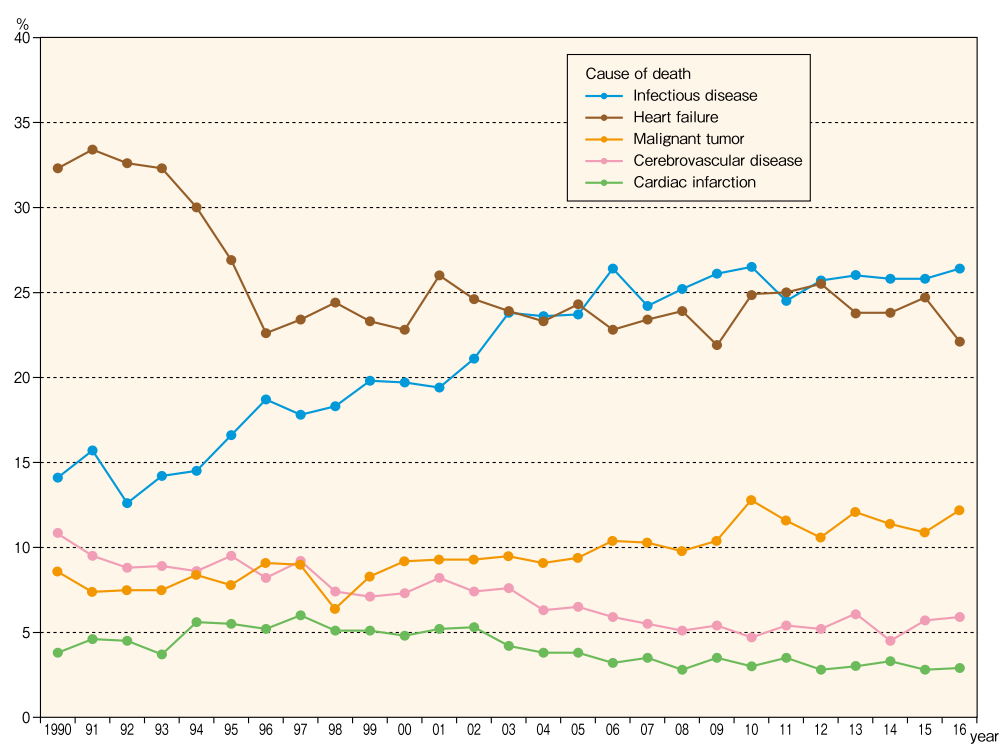

Fig. 15 Major causes of death during the incident year, 1990-2016 


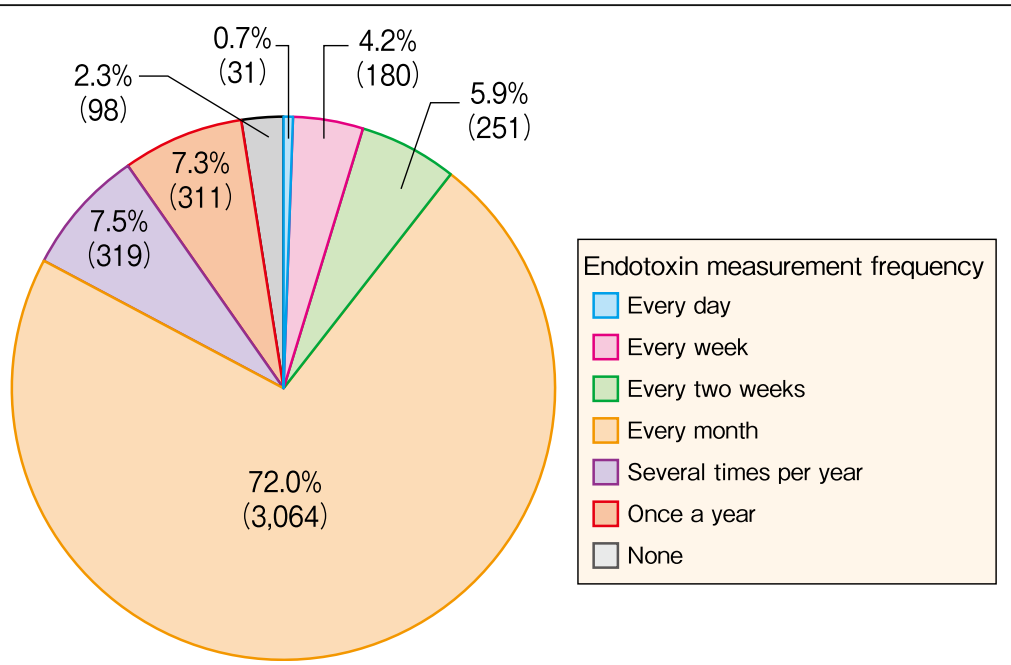

Fig. 16 Facility distribution, by endotoxin measurement frequency, 2016

Diabetic nephropathy, which continued to increase in prevalence, is now the most common primary disease since supplanting chronic glomerulonephritis in the 2011 JRDR. Although the prevalence of diabetic nephropathy has continued to increase since 2011, the pace of this increase has slowed somewhat in recent years (Fig. 8, Additional file 8: Table S8). The percentage of chronic glomerulonephritis has been decreasing linearly, whereas the percentages of nephrosclerosis and unknown primary disease have continued to increase. In addition, the percentages of primary diseases such as polycystic kidney disease, chronic pyelonephritis, lupus nephritis, and rapidly progressive glomerulonephritis have remained at approximately the same levels as in previous years.

\section{Causes of death among deceased patients in 2016}

In the 2016 facility survey, 31,790 deaths were reported; among these, the sex of the patients and the cause of death were listed in the patient survey for 30,638 patients (96.4\%). Among men, the most common cause of death was heart failure (25.1\%), followed by infectious disease (22.5\%), malignant tumor (10.6\%), and cerebrovascular disease (6.3\%). Among women, the most common cause of death was heart failure (26.9\%), followed by infectious disease (20.8\%), malignant tumor (7.9\%), and cerebrovascular disease $(6.8 \%)$. For women, the ranking positions of malignant tumor and cerebrovascular disease were switched from those in 2015. Among all deceased patients, the most common cause of death was

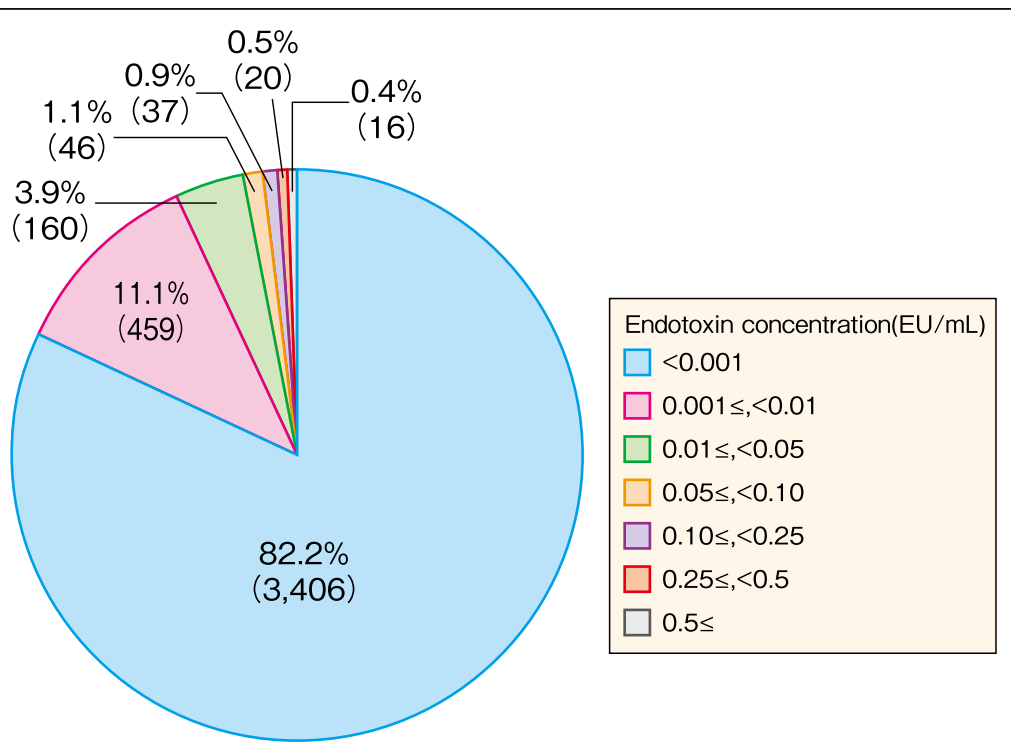

Fig. 17 Facility distribution, by endotoxin concentration, 2016 


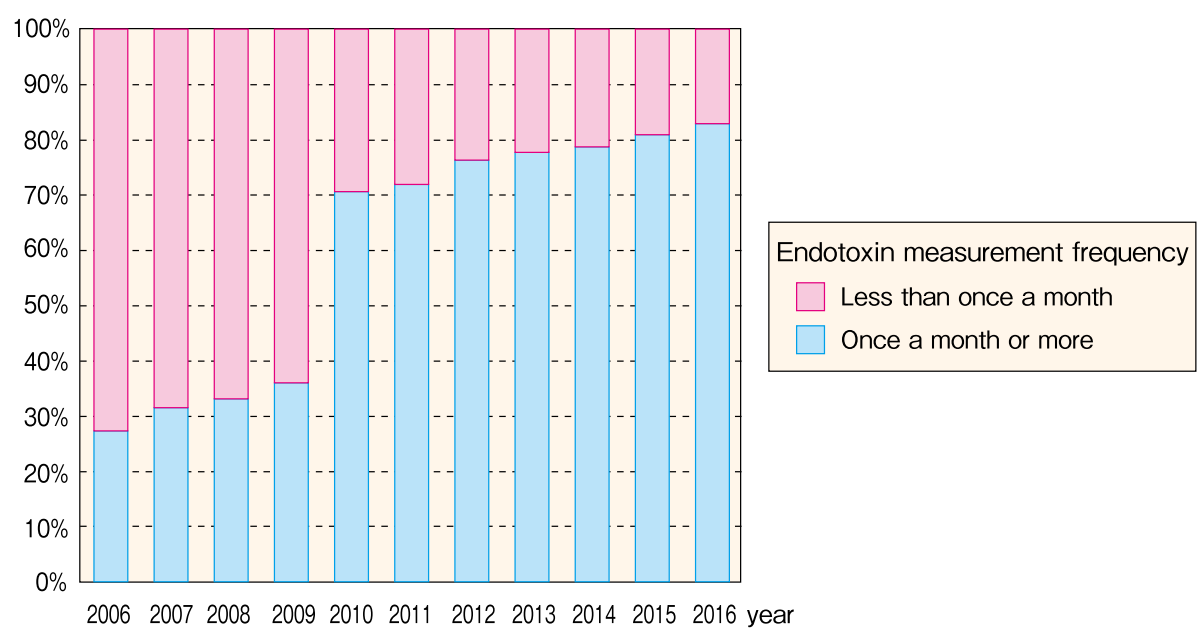

Fig. 18 Facility distribution, by endotoxin measurement frequency, 2006-2016

heart failure (25.7\%), followed by infectious disease (21.9\%), malignant tumor (9.7\%), and cerebrovascular disease (6.5\%). The orders of causes of death by sex and for all patients were unchanged from those in 2015. Cardiovascular disease (heart failure, cerebrovascular disease, and myocardial infarction) accounted for $35.6 \%$ of deaths among men, 37.1\% among women, and 36.2\% overall (Fig. 9, Additional file 9: Table S9).

Concerning changes in the overall causes of death over time, the most common cause of death is heart failure, which has accounted for $26-27 \%$ of deaths in the past several years. Death due to infectious disease had been consistently increasing since around 1993 but decreased by 0.2 percentage points from 2015 to 2016. Cerebrovascular disease has been consistently gradually decreasing since 1994. Recently, myocardial infarction-related deaths have tended to gradually decrease since peaking at $8.4 \%$ in 1997 . Deaths due to malignant tumors have gradually increased from a low of $5.8 \%$ at the end of 1987 but have remained at $9-10 \%$ since reaching that point in 2004. Cardiovascular disease (heart failure, cerebrovascular disease, and myocardial infarction) accounted for $54.8 \%$ of deaths in 1988; however, this figure has been decreasing at a nearly constant pace, reaching $36.1 \%$ in 2016 (Fig. 10, Additional file 10: Table S10). The causeof-death category codes in the JRDR were substantially revised in the 2003 and 2010 surveys. Please refer to the 2010 JRDR report for the details of these revisions [11].

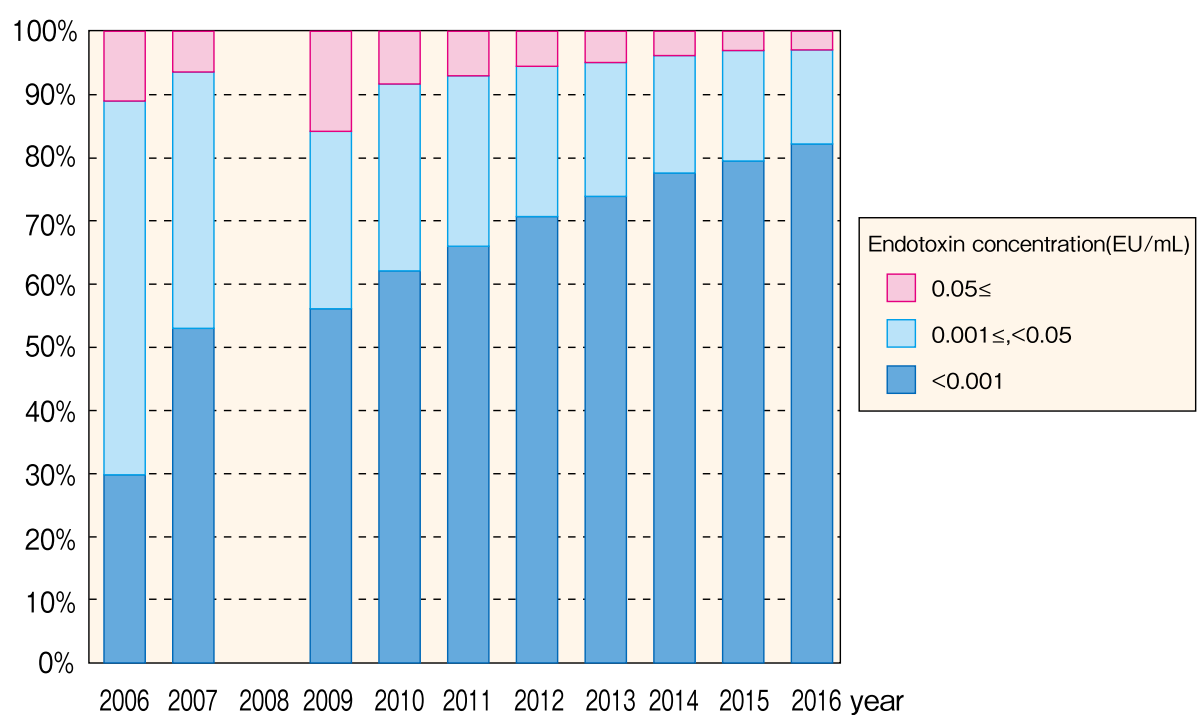

Fig. 19 Facility distribution, by endotoxin concentration, 2006-2016. The unit of endotoxin in the questionnaire has changed in 2008. The data of the year were omitted because of the potentially higher rate of erroneous results 


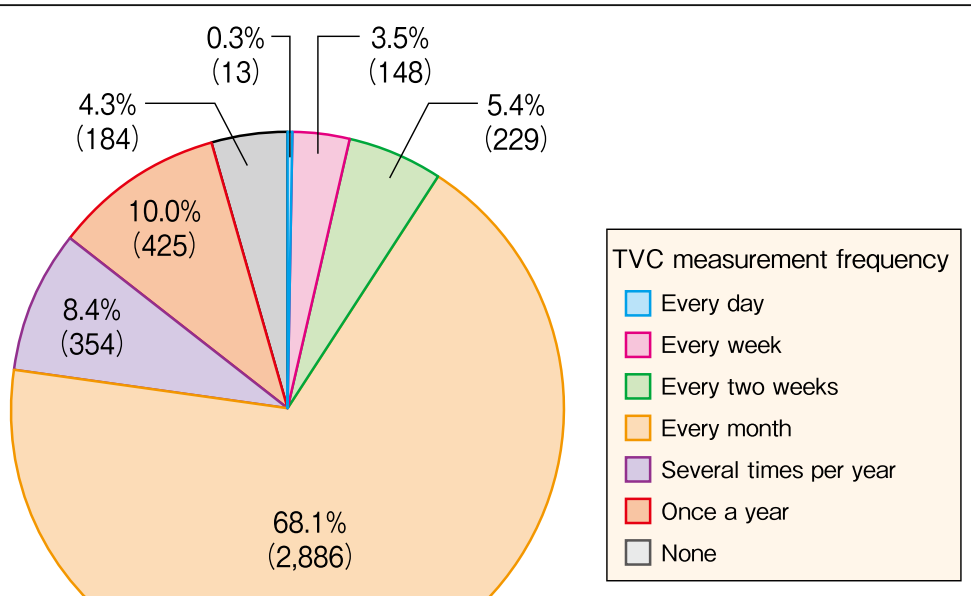

TVC: Total viable microbial count

Fig. 20 Facility distribution, by TVC measurement frequency, 2016. Abbreviation: TVC total viable microbial count

\section{Crude death rate}

We calculated the annual crude death rate based on the patient dynamics in the facility survey, as follows:

Crude death rate

$=[$ number of deaths $/$ (previous year patient count + survey year patient count) $/ 2] \times 100(\%)$.

Because incident patients increased in age and included greater numbers of patients with poor prognosis owing to increases in diabetic nephropathy, nephrosclerosis, and similar diseases, the crude death rate has tended to worsen every year. The lowest crude death rate was $7.9 \%$ in 1989 , a year with a low survey response rate. However, the crude death rate has remained at 9.2$10.2 \%$ since 1992 , when it reached $9.7 \%$ and thus exceeded 9\% for the first time; in 2016, the crude death rate was $9.7 \%$ (Table 6).

\section{Chapter 2: 2016 incident dialysis patient dynamics}

Age, sex, and dialysis vintage of incident patients in 2016

In the 2016 patient survey, the count of incident dialysis patients with confirmed age and sex was 37,250, which is 2094 patients fewer than, or $94.7 \%$ of, the 39,344 patients noted in the facility survey. There were 25,506 men and 11,744 women; as in the previous year, there were approximately twice as many men as women. The mean age of all incident patients was 69.40 years, a 0.20 -year increase from the mean age of 69.20 years at the end of 2015. The mean age of men and women was 68.57 and 71.19 years, respectively, which represent increases of 0.20 and

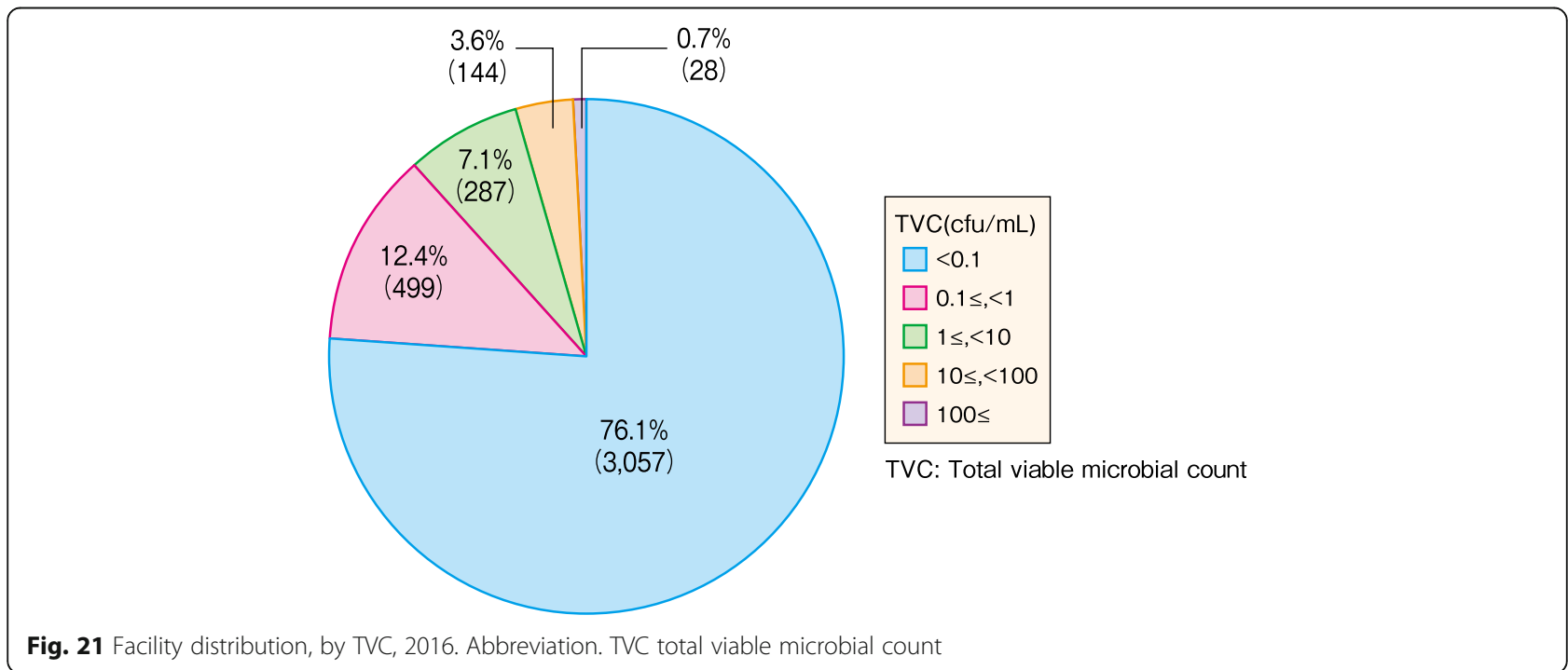




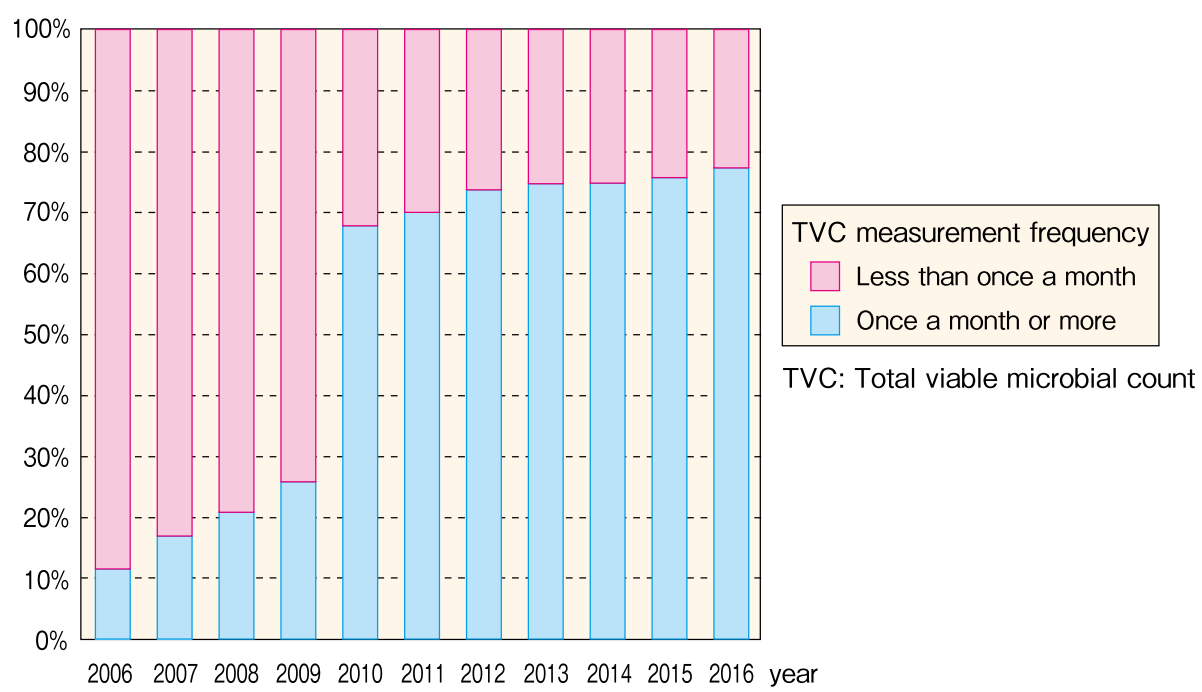

Fig. 22 Facility distribution, by TVC measurement frequency, 2006-2016. Abbreviation: TVC total viable microbial count

0.24 years from the previous year, respectively. The most common 5-year age groups among incident patients were 65-69 years for men and 80-84 years for women. Old-old patients (75 years and older) accounted for $47.2 \%$ of women and $37.3 \%$ of men (Fig. 11, Additional file 11: Table S11).

The mean age of incident dialysis patients has been increasing nearly linearly each year, but the rate of increase has been slowing. The mean age of incident patients in 2016 was 69.4 years, which is 0.2 years higher than that in the previous year (Table 7).

\section{Primary diseases of incident patients in 2016}

The most prevalent primary disease among incident dialysis patients in 2016 was diabetic nephropathy, (43.2\% of patients), followed by chronic glomerulonephritis (16.6\%) and nephrosclerosis (14.2\%); the primary disease was unknown for $12.8 \%$ of patients. The prevalence of diabetic nephropathy differed between men (46.2\%) and women (36.7\%). No sex-based difference was observed for chronic glomerulonephritis or nephrosclerosis. Although male patients outnumbered female patients overall, there were more female than male patients with lupus nephritis and amyloidosis (Fig. 12, Additional file 12: Table S12).

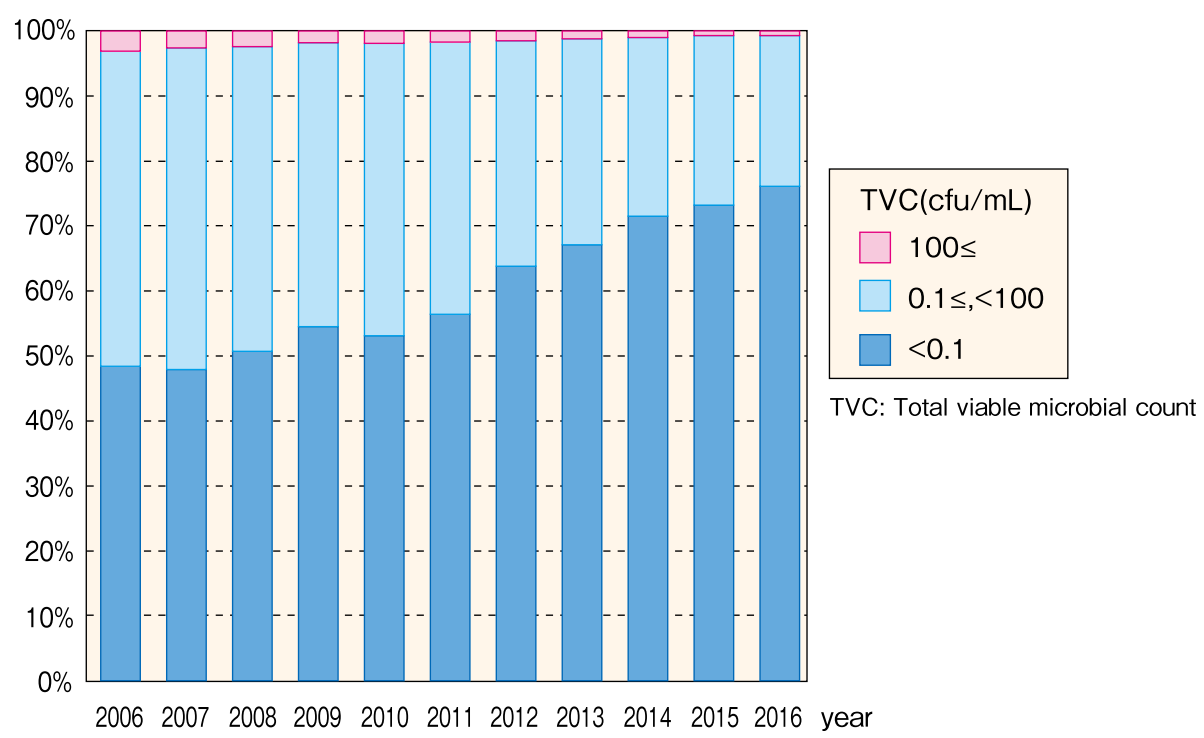

Fig. 23 Facility distribution, by TVC, 2006-2016. Abbreviation: TVC total viable microbial count 


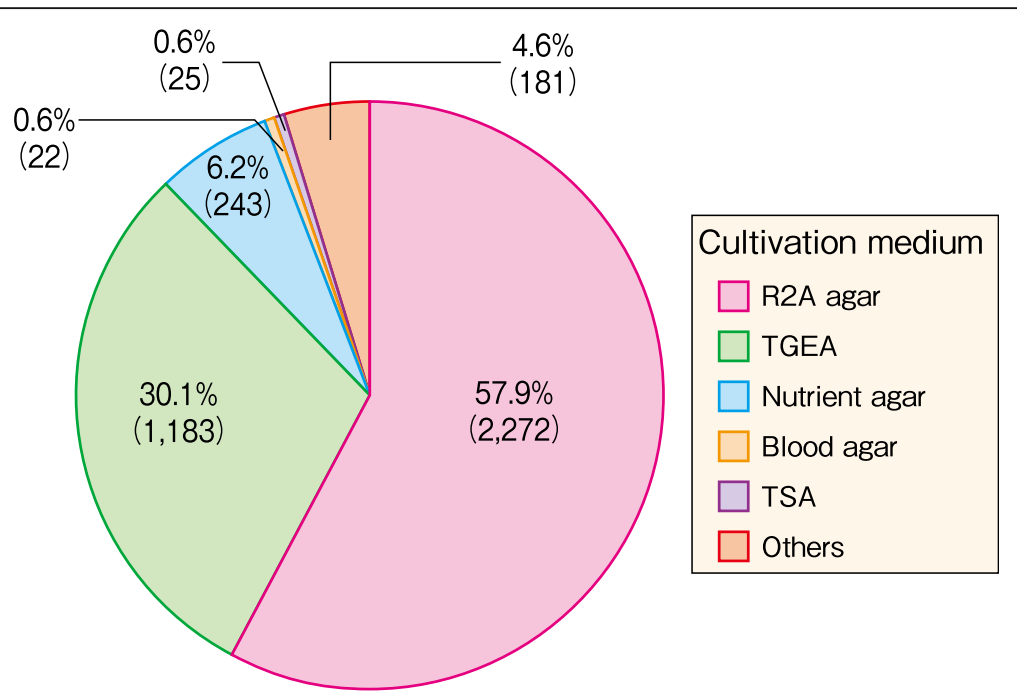

Fig. 24 Facility distribution on TVC measurement, by cultivation medium, 2016. Abbreviations: R2A Reasoner's no. 2 agar, TGEA tryptone glucose extract agar, TSA trypticase soy agar, TVC total viable microbial count

In 1998, diabetic nephropathy supplanted chronic glomerulonephritis as the most common primary disease among incident patients; the distribution of diabetic nephropathy has increased consistently ever since, but it has remained nearly the same in the past few years. Chronic glomerulonephritis has been consistently decreasing, whereas the unknown primary disease has been consistently increasing (Fig. 13, Additional file 13: Table S13).

\section{Causes of death among incident patients in 2016}

The most common cause of death among the overall incident dialysis patients in 2016 was infectious disease (26.4\%), followed by heart failure $(22.1 \%)$ and malignant cancer (12.2\%). Among male incident dialysis patients was infectious disease (27.4\%), followed by heart failure (21.4\%), malignant tumor (12.7\%), and others (10.0\%). Among female incident dialysis patients, the most common cause of death was infectious disease (24.4\%), followed by heart failure (23.5\%), others $(12.7 \%)$, and malignant tumor (11.1\%) (Fig. 14, Additional file 14: Table S14).

In the 1990s, the most common cause of death among incident dialysis patients was heart failure. However, the distribution of infectious disease increased gradually, reaching and surpassing the percentage of heart failure during the year 2000. In 2016, infectious disease remained the most common cause of death among incident dialysis patients (26.4\%), followed by heart failure $(22.1 \%)$. The subsequent order of malignant tumor

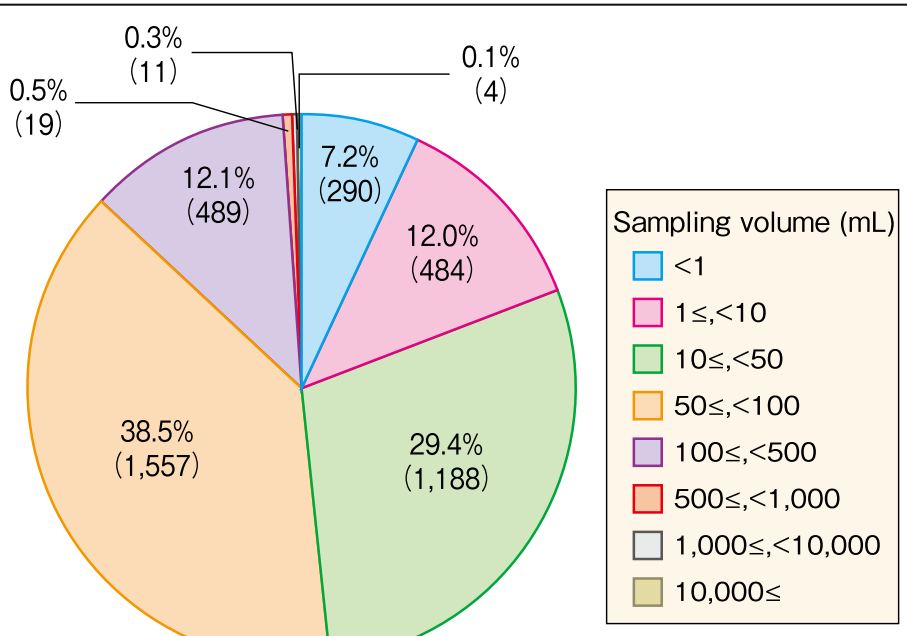

TVC: Total viable microbial count

Fig. 25 Facility distribution on TVC measurement, by sampling volume, 2016. Abbreviation: TVC total viable microbial count 


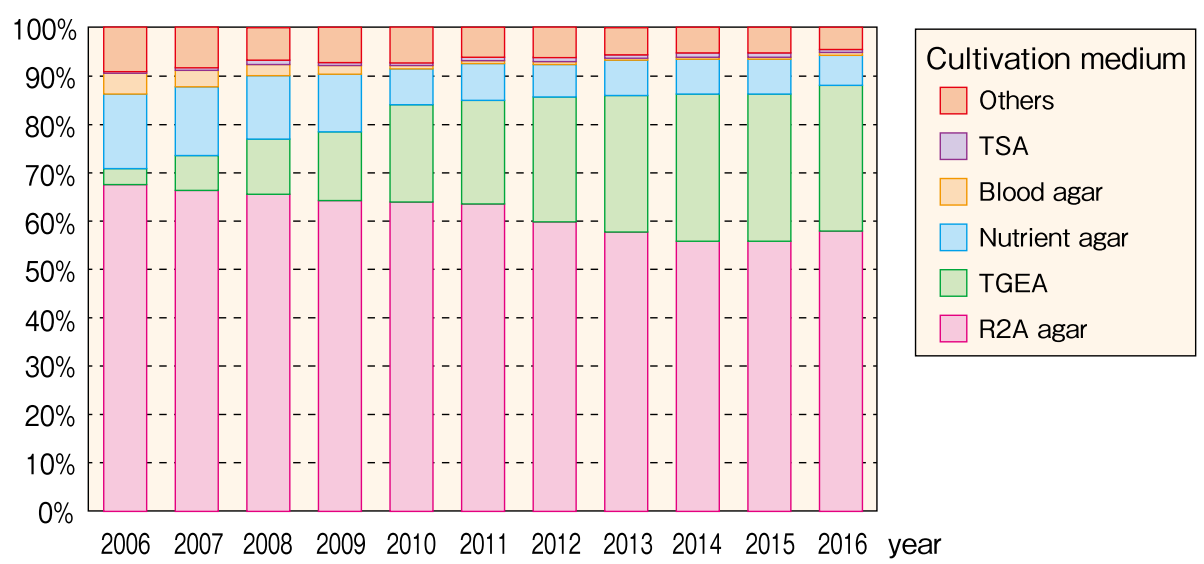

Fig. 26 Facility distribution, by cultivation medium, 2006-2016. Abbreviations: R2A Reasoner's no. 2 agar, TGEA tryptone glucose extract agar, TSA trypticase soy agar

(12.2\%), cerebrovascular disease (5.9\%), and myocardial infarction (2.9\%) has remained unchanged. Over the long term, the percentages of deaths due to cerebrovascular disease and myocardial infarction have been declining, whereas the percentages of deaths due to infectious disease and malignant tumor have been increasing (Fig. 15, Additional file 15: Table S15).

\section{Chapter 3: Dialysis fluid quality management Overview}

Since 2006, the JRDR has conducted a survey on microbiological quality and management of dialysis fluid. Based on the results, the standards for dialysis fluid microbiological quality were revised in 2008 [12], and further standards for biochemical contamination were added in 2016 [13]. In these standards, dialysis fluid microbiological quality is recommended to be assessed both by ET concentration and TVC, which are both evaluated a minimum of once monthly. At least one dialysis console is tested each month, and all consoles are tested a minimum of once annually. The required minimum quality for standard dialysis fluid used in dialysis therapy was defined as an ET concentration of $<0.05$ $\mathrm{EU} / \mathrm{mL}$ and a TVC of $<100 \mathrm{cfu} / \mathrm{mL}$. Furthermore, we recommended the use of ultrapure dialysis fluid (UPD) for all dialysis therapy; UPD is defined as having an ET concentration of $<0.001 \mathrm{EU} / \mathrm{mL}$ (less than the measurement sensitivity) and a TVC of $<0.1 \mathrm{cfu} / \mathrm{mL}$. At the time these standards were adopted, as well as in 2016, they were the strictest quality standards for dialysis fluid worldwide. Furthermore, in the 2010 revision of the medical reimbursement system, dialysis fluid quality was added; as a result, dialysis fluid quality control dramatically improved after the 2010 JRDR [10]. In 2015, dialysis fluid ET concentration and patient prognosis were analyzed using JRDR data; the patient group being treated at facilities with a dialysis fluid ET concentration $<0.001$ $\mathrm{EU} / \mathrm{mL}$ was found to have a higher 1-year survival rate than the patient group undergoing treatment at facilities with a concentration of $\geq 0.100 \mathrm{EU} / \mathrm{mL}$ [14].

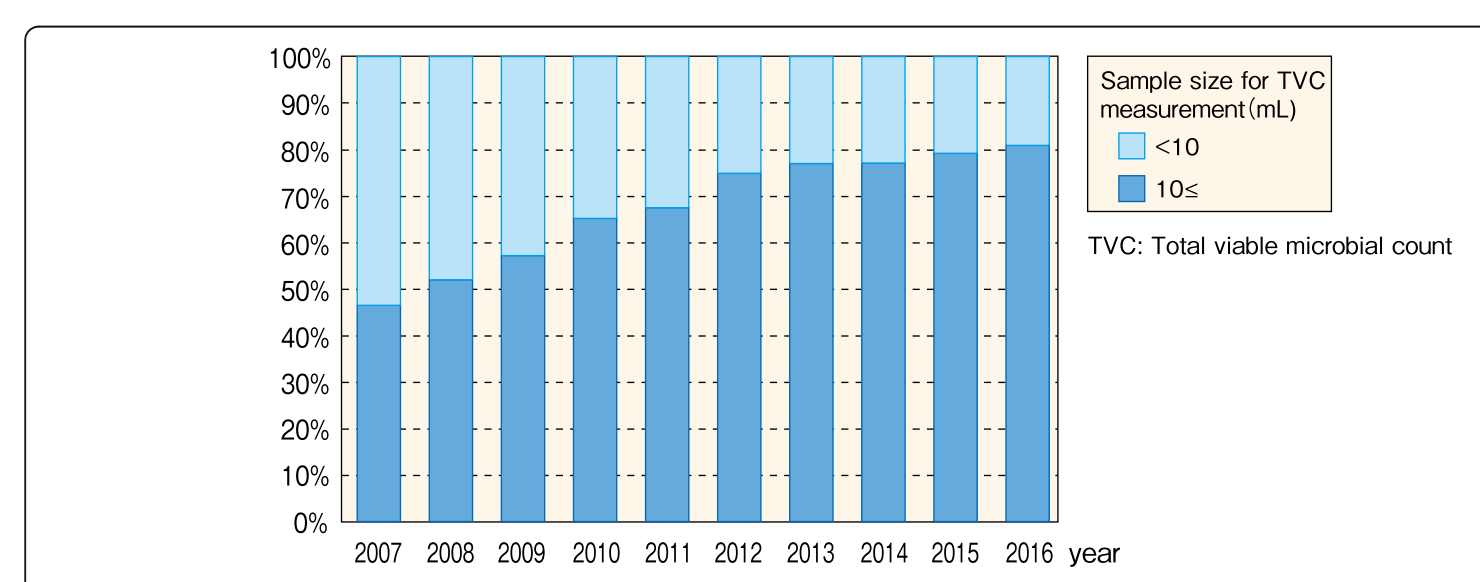

Fig. 27 Facility distribution, by sampling volume for TVC measurement, 2006-2016. Abbreviation: TVC total viable microbial count 
Table 8 Facility counts, by ETRF installation, 2016

\begin{tabular}{|c|c|c|c|c|c|}
\hline & With ETRF & Without ETRF & Subtotal & No information available & Total \\
\hline Number of facilities (\%) & $4204(97.4)$ & $112(2.6)$ & $4316(100.0)$ & 2 & 4318 \\
\hline
\end{tabular}

Values in parentheses under each figure represent the percentage relative to the subtotal in the row. The above data were obtained from the facility survey Abbreviations: ETRF endotoxin retentive filter

\section{Dialysis fluid endotoxin testing}

According to the JSDT standard, dialysis fluid endotoxin (ET) concentration is assayed using Limulus tests [12]. In Japan, these ET assay systems are commercially available at a relatively low cost and widely used. However, this situation is rather rare globally.

A total of 4318 facilities had at least one dialysis console; 4254 of these facilities (98.5\%) stated their dialysis fluid ET test frequency. Of these 4254 facilities, 3526 facilities $(82.9 \%)$ met the JSDT standard of testing dialysis fluid for ET at least once monthly (Fig. 16, Additional file 16: Table S16). Responses about dialysis fluid ET concentration were obtained from 4144 facilities (96.0\%). A total of 3406 facilities (82.2\%) reached a concentration of $<0.001 \mathrm{EU} / \mathrm{mL}$, which qualifies as UPD, and 4025 facilities $(97.1 \%)$ reached a concentration of $<$ $0.050 \mathrm{EU} / \mathrm{mL}$, which qualifies as standard dialysis fluid (Fig. 17, Additional file 16: Table S16).

In 2008, when the JSDT standards for dialysis fluid were enacted, only $33.1 \%$ of dialysis fluid ET tests met these standards; however, this percentage improved to $70.6 \%$ in 2010 , when payments for dialysis fluid quality management were added to the medical reimbursement system, and has been gradually increasing since (Fig. 18, Additional file 17: Table S17). The levels of dialysis fluid ET concentration qualifying as UPD and as standard dialysis fluid have both increased over time (Fig. 19, Additional file 18: Table S18). The absence of values for dialysis fluid ET concentration in 2008 is due to the switch in the unit of dialysis fluid ET concentration from EU/L to EU/mL based on international rules in the survey that year, resulting in many incorrect entries.

\section{Dialysis fluid total viable microbial count testing}

The JSDT standards for dialysis fluid stipulate that dialysis fluid total viable microbial count (TVC) is to be assessed as the number of bacterial colonies formed after culturing for 7 days at $17-23^{\circ} \mathrm{C}$ on a heterotrophic agar plate medium, as described below [12]. A total of
4239 facilities (98.1\%) stated how frequently they assay the TVC of dialysis fluid; of these 4239 facilities, 3276 facilities (77.3\%) met the JSDT standard of performing assays at least once per month (Fig. 20, Additional file 19: Table S19). A total of 4015 facilities (93.0\%) stated the TVC of their dialysis fluid; of these 4015 facilities, 3057 facilities $(76.1 \%)$ reached a TVC of $<0.1 \mathrm{cfu} / \mathrm{mL}$, which qualifies as UPD, and 3987 facilities (99.3\%) reached a TVC of $<100 \mathrm{cfu} / \mathrm{mL}$, which qualifies as standard dialysis fluid (Fig. 21, Additional file 19: Table S19).

The frequency of TVC testing has increased over time; although this frequency improved in 2010, as did the frequency of ET assays, TVC testing has always been performed slightly less frequently than ET assays (Fig. 22, Additional file 20: Table S20). As with dialysis fluid ET concentrations, the levels of TVC qualifying as UPD and as standard dialysis fluid have increased over time (Fig. 23, Additional file 21: Table S21).

As described above, the JSDT standards for dialysis fluid recommend the use of a certified bacterial culture medium such as Reasoner's no. 2 agar (R2A), tryptone glucose extract agar (TGEA), or one with a similar sensitivity [12]. In general, in methods using an agar plate medium, a $0.5-\mathrm{mL}$ sampling volume is the minimum volume that guarantees a TVC of $100 \mathrm{cfu} / \mathrm{mL}$, which qualifies as standard dialysis fluid. However, the UPD standard is $<0.1 \mathrm{cfu} / \mathrm{mL}$; to meet this standard, a minimum of $10 \mathrm{~mL}$ dialysis fluid must be sampled and then cultured after being strained through a membrane filter. Therefore, the JRDR examines both the culture medium and the sampling volume.

In the 2015 JRDR, 3926 of 4318 facilities (91.0\%) stated the medium they used for TCV testing. Of these facilities, $57.9 \%$ used R2A and $30.1 \%$ used TGEA; altogether, $88.0 \%$ of the facilities met the JSDT standard (Fig. 24, Additional file 22: Table S22). Of 4303 facilities, 4042 (93.9\%) stated the dialysis fluid sampling volume used for TVC testing. In the 2016 survey, $80.9 \%$ of facilities sampled $10 \mathrm{~mL}$ or more dialysis fluid, thereby qualifying as UPD (Fig. 25, Additional file 22: Table S22).

Table 9 Dialysis console counts, by ETRF installation, 2016

\begin{tabular}{|c|c|c|c|c|c|}
\hline & \multicolumn{2}{|c|}{ Facility status of ETRF installation } & \multirow[t]{2}{*}{ Subtotal } & \multirow{2}{*}{$\begin{array}{l}\text { No } \\
\text { information } \\
\text { available }\end{array}$} & \multirow[t]{2}{*}{ Total } \\
\hline & Dialysis consoles with ETRF & Dialysis consoles without ETRF & & & \\
\hline Numbers of dialysis consoles (\%) & $124,705(92.3)$ & $10,423(7.7)$ & $135,128(100.0)$ & 83 & 135,211 \\
\hline
\end{tabular}




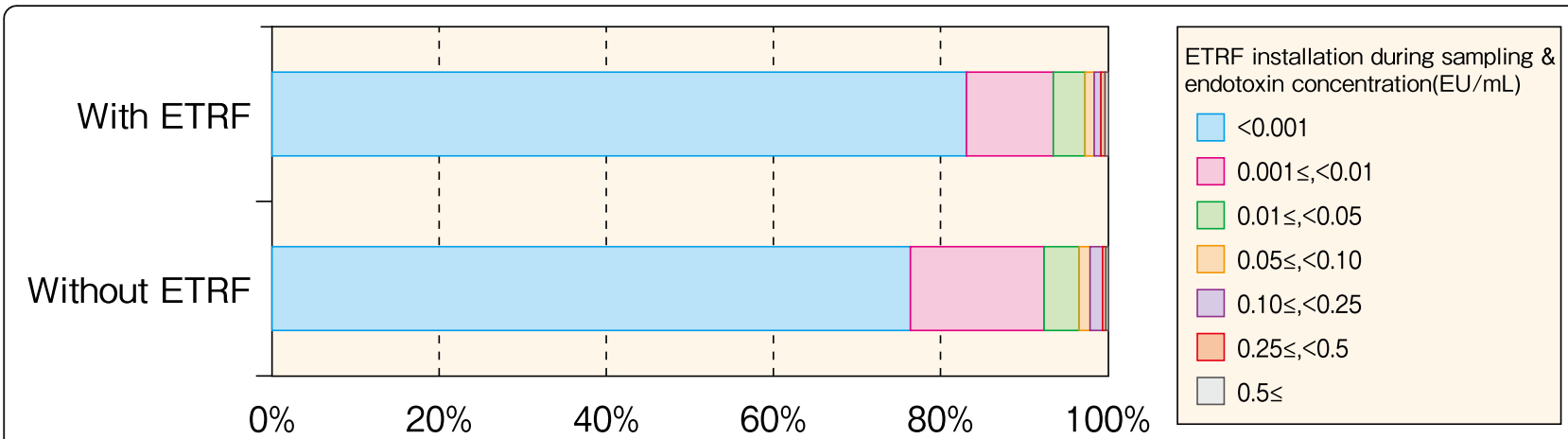

Fig. 28 Facility distribution, by ETRF installation during sampling endotoxin concentration, 2016. Abbreviation: ETRF endotoxin retentive filter

The use of R2A has been decreasing over time, whereas the use of TGEA has been increasing. Thus, the percentage of facilities that meet the JSDT standard has increased overall (Fig. 26, Additional file 23: Table S23). The percentage of facilities that use a level of sampling volume that qualifies as UPD has also been increasing over time (Fig. 27, Additional file 24: Table S24).

\section{Use of endotoxin retentive filters (ETRFs)}

The appropriate use of an ETRF is essential for preparing dialysis fluid that remains consistently clean. To that end, the JSDT enacted a standard for ETRF management in 2011 [15].

Of the 4318 facilities with at least one dialysis console, 4316 facilities (99.9\%) provided responses about ETRF installation. Among these 4316 facilities, 4204 facilities (97.4\%) had installed an ETRF in one or more dialysis consoles. Of the 135,211 dialysis consoles installed at the abovementioned 4316 facilities, 124,705 consoles (92.3\%) had an ETRF installed (Tables 8 and 9). The percentage of facilities meeting the UPD standard (ET concentration $<0.001 \mathrm{EU} / \mathrm{mL}$ and TVC $<0.1 \mathrm{cfu} / \mathrm{mL}$ ) was higher among facilities that use an ETRF during sampling than those that do not (Fig. 28, Additional file 25: Table S25, Fig. 29, Additional file 26: Table S26). In general, unless the contamination of dialysis fluid immediately before the ETRF is extremely high, it is theoretically possible to reach the UPD standard for both ET and TVC by passing the dialysis fluid through the ETRF once. However, despite the use of an ETRF, the UPD standards for ET concentration and TVC were not met by 16.9 and $22.7 \%$ of the facilities, respectively. These results suggest that although the spread of ETRF use has contributed to the cleanliness of dialysis fluid, there are still issues, such as the handling of ETRFs, that inhibit the achievement of the UPD standard [16].

\section{Overall evaluation of the microbiological quality of dialysis fluid}

The JSDT standard stipulates that for the microbiological quality of dialysis fluid to qualify as UPD or standard dialysis fluid, the specified levels for both dialysis fluid ET concentration and TVC must be met simultaneously [11, 12]. Of 4318 facilities, 4008 (92.8\%) stated both their dialysis fluid ET concentration and TVC. These included 2863 facilities (71.4\%) that achieved UPD status and 3888 facilities (97.0\%) that achieved standard dialysis fluid status (Fig. 30, Additional file 27: Table S27). The rates of achievement of the UPD and

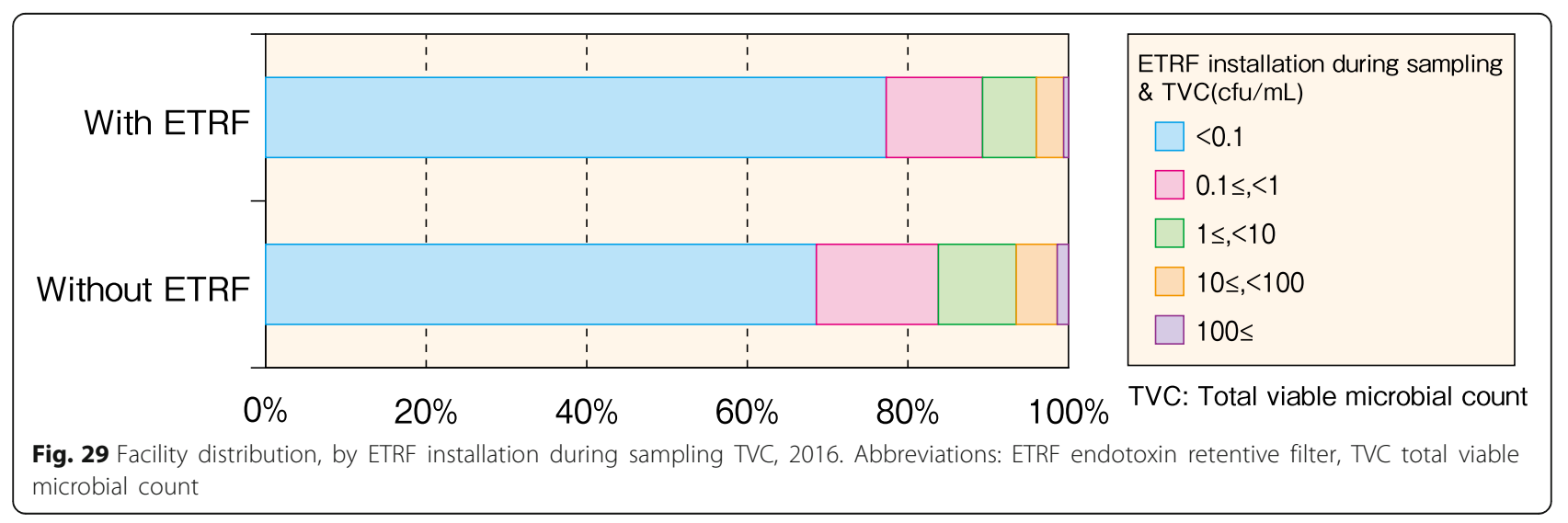




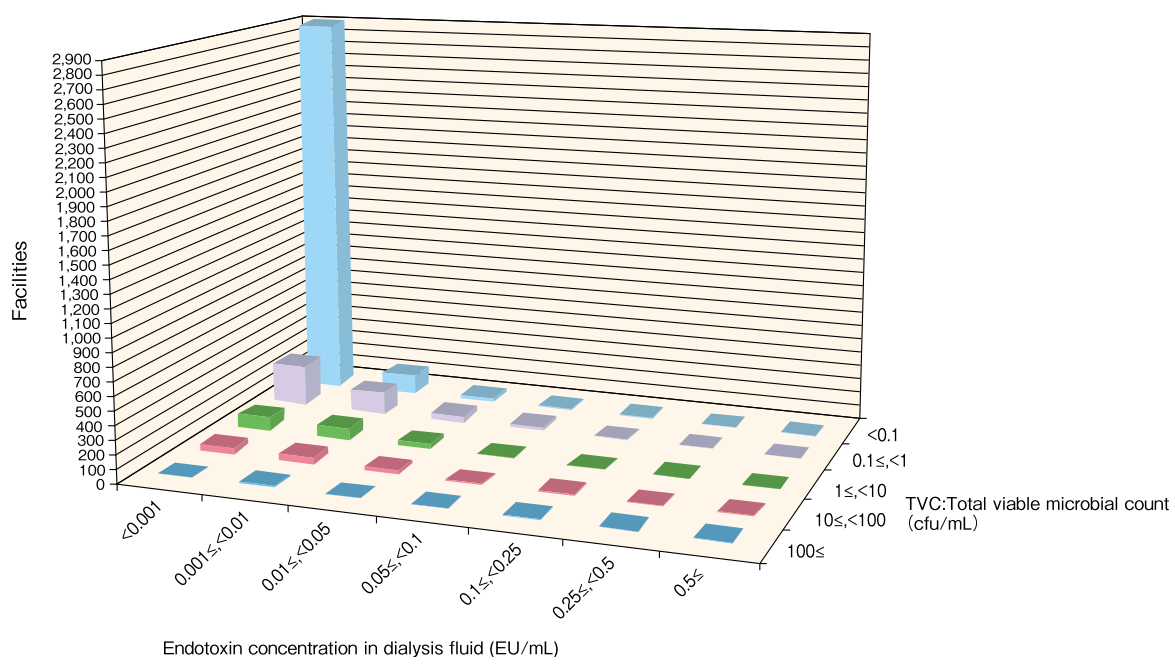

Fig. 30 Facility distribution, by TVC and endotoxin concentration, 2016. Abbreviation: TVC total viable microbial count

standard dialysis fluid status have been improving over time (Fig. 31, Additional file 28: Table S28).

\section{Chapter 4: hemodiafiltration (HDF)}

\section{Hemodiafiltration patient dynamics}

HDF includes on-line HDF, off-line HDF, push/pull $\mathrm{HDF}$, acetate-free biofiltration (AFBF), and intermittent infusion hemodiafiltration (IHDF). The number of HDF patients in Japan has rapidly increased in recent years and had reached 74,799 patients by the end of 2016, accounting for $24.2 \%$ of all HD/HDF patients (Fig. 32, Additional file 29: Table S29). Of these 74,799 HDF patients, 48,291 (64.6\%) were men and 26,508 (35.4\%) were women (Table 10). The mean age was 65.4 years for men and 67.5 years for women, and the most common age group for both men and women was 65-69 years. These distributions and trends resembled those of HD patients, and it was shown that HDF was being performed for a wide range of ages. The distribution of dialysis vintages was largely the same as that for HD patients (Table 11). Men tended to be more numerous in each age group, although the numbers of male and female patients with dialysis vintages of 25 years or longer were nearly identical. The major primary diseases of HDF patients were diabetic nephropathy (35.8\%) and chronic glomerulonephritis (32.8\%). Thus, as with HD patients, the percentage of diabetic nephropathy was high, whereas the percentage of chronic glomerulonephritis was low (Table 12).

\section{HDF modalities}

The most common HDF modality in 2016 was on-line HDF, which accounted for 59,116 patients $(79.0 \%$ of all HDF patients) (Fig. 32, Additional file 29: Table S29). Until 2011, the major HDF modality was off-line HDF; however, since 2012, on-line HDF has been the major

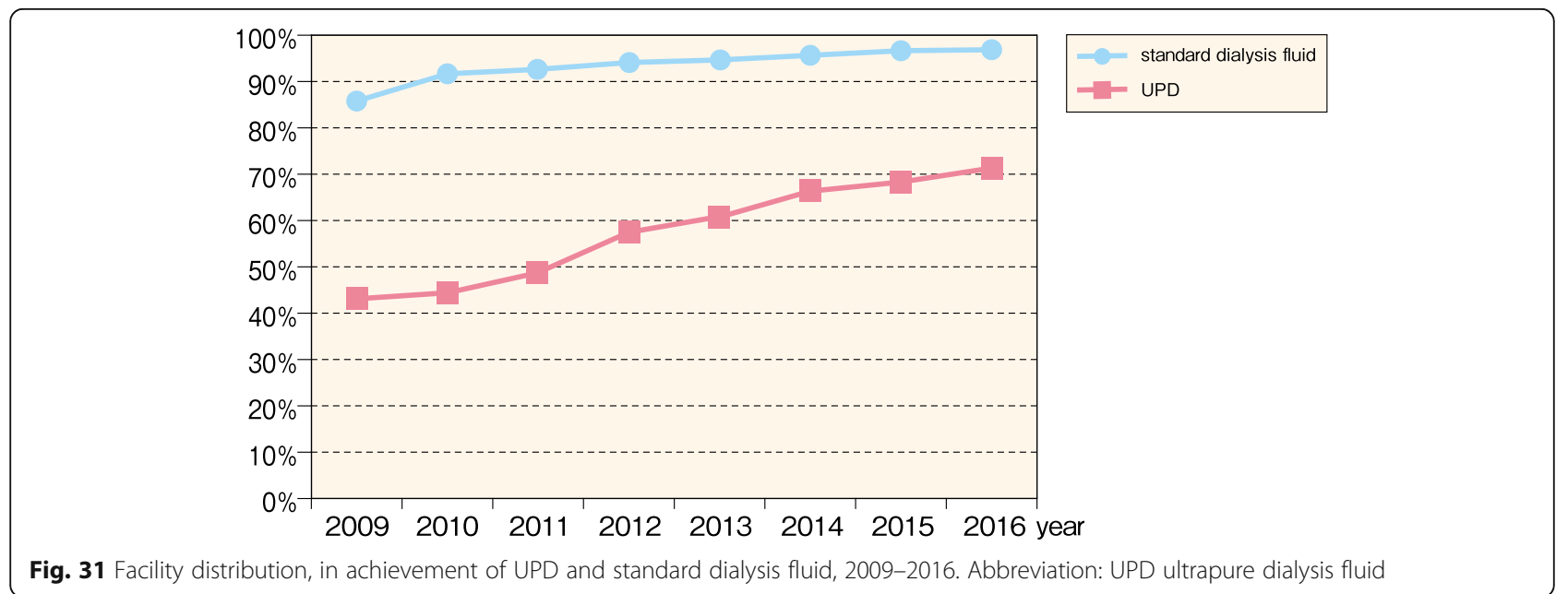




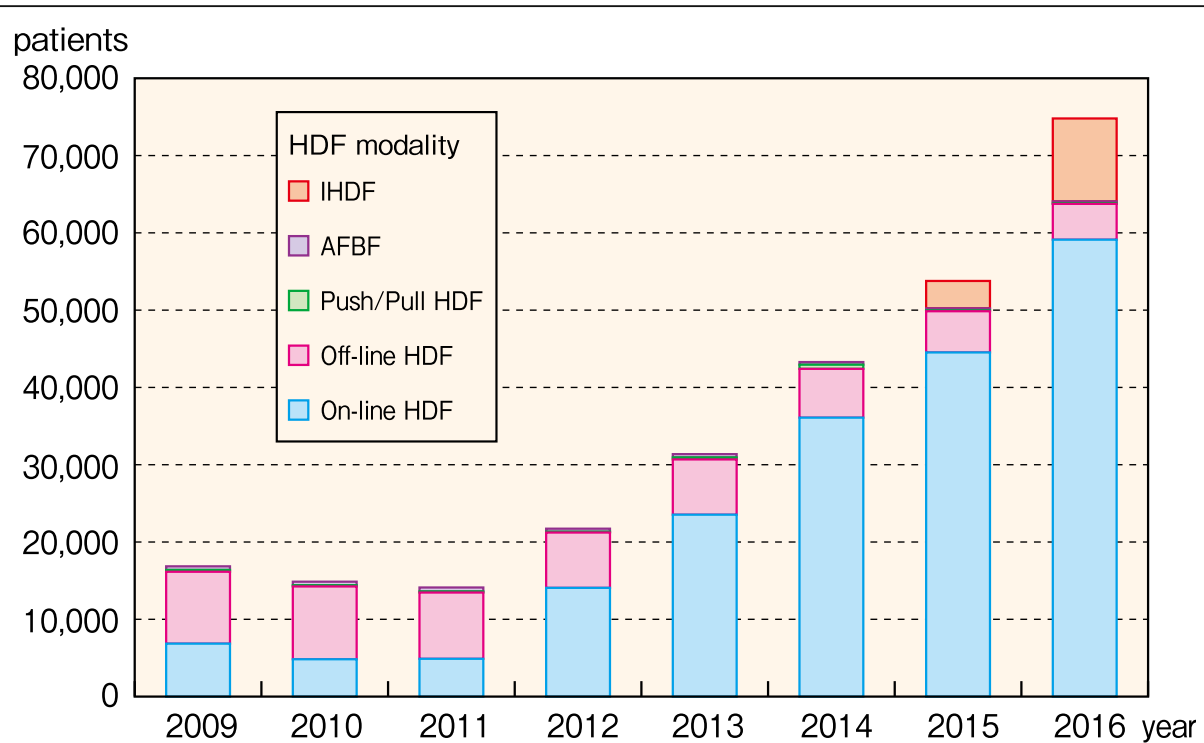

Fig. 32 HDF patient counts, by HDF modality, 2009-2016. Abbreviations: AFBF acetate-free biofiltration, HDF hemodiafiltration, IHDF intermittent infusion hemodiafiltration

modality and its use has increased tremendously. In contrast, the number of off-line HDF patients has been decreasing yearly. In 2015, IHDF was added to the survey items and has been trending upward, with 10,728 patients at the end of 2016 (14.3\% of all HDF patients).

Among all patients and all dialysis modalities (including detailed HDF modalities), the percentage of HDF tended to increase as the dialysis vintage lengthened (Fig. 33, Additional file 30: Table S30). The percentages of both on-line and off-line HDF tended to increase as the dialysis vintage lengthened. Among all the groups, IHDF accounted for about $2-3 \%$ of all patients. As the dialysis vintage lengthened, the percentage of PD decreased, whereas the percentage of hemoadsorption dialysis tended to increase; hemoadsorption dialysis was performed in $11.5 \%$ of all patients with dialysis vintages of at least 40 years.

\section{HDF prescriptions}

The HDF prescriptions consisted of the four possible combinations of on-line/off-line HDF and the dilution method (pre-dilution and post-dilution). The most common combination was on-line HDF/pre-dilution (48,457 patients),

Table 10 HDF patient distribution, by age \& sex, 2016

\begin{tabular}{|c|c|c|c|c|c|c|c|c|c|c|}
\hline sex & $<20$ & $20 \leq,<25$ & $25 \leq,<30$ & $30 \leq,<35$ & $35 \leq,<40$ & $40 \leq,<45$ & $45 \leq,<50$ & $50 \leq,<55$ & $55 \leq,<60$ & $60 \leq,<65$ \\
\hline Male & 7 & 31 & 126 & 349 & 766 & 1,684 & 2,958 & 3,719 & 4,623 & 6,192 \\
\hline (\%) & $(0.0)$ & $(0.1)$ & $(0.3)$ & $(0.7)$ & (1.6) & (3.5) & (6.1) & $(7.7)$ & (9.6) & $(12.8)$ \\
\hline Female & 4 & 22 & 65 & 141 & 308 & 750 & 1,203 & 1,612 & 2,218 & 3,213 \\
\hline (\%) & $(0.0)$ & $(0.1)$ & $(0.2)$ & $(0.5)$ & $(1.2)$ & $(2.8)$ & $(4.5)$ & $(6.1)$ & $(8.4)$ & $(12.1)$ \\
\hline Total & 11 & 53 & 191 & 490 & 1,074 & 2,434 & 4,161 & 5,331 & 6,841 & 9,405 \\
\hline (\%) & $(0.0)$ & $(0.1)$ & $(0.3)$ & $(0.7)$ & $(1.4)$ & (3.3) & $(5.6)$ & $(7.1)$ & $(9.1)$ & $(12.6)$ \\
\hline sex & $65 \leq,<70$ & $70 \leq,<75$ & $75 \leq,<80$ & $80 \leq,<85$ & $85 \leq,<90$ & $90 \leq,<95$ & $95 \leq$ & Total & Mean age & S.D. \\
\hline Male & 9,153 & 6,761 & 5,775 & 3,937 & 1,739 & 434 & 37 & 48,291 & 65.42 & 12.45 \\
\hline (\%) & (19.0) & $(14.0)$ & $(12.0)$ & $(8.2)$ & (3.6) & $(0.9)$ & $(0.1)$ & 100 & & \\
\hline Female & 5,049 & 3,866 & 3,538 & 2,575 & 1,458 & 439 & 47 & 26,508 & 67.49 & 12.42 \\
\hline (\%) & (19.0) & (14.6) & (13.3) & $(9.7)$ & $(5.5)$ & $(1.7)$ & $(0.2)$ & 100 & & \\
\hline Total & 14,202 & 10,627 & 9,313 & 6,512 & 3,197 & 873 & 84 & 74,799 & 66.15 & 12.48 \\
\hline (\%) & (19.0) & $(14.2)$ & (12.5) & $(8.7)$ & (4.3) & $(1.2)$ & $(0.1)$ & (100.0) & & \\
\hline
\end{tabular}


Table 11 HDF patient distribution, by sex and dialysis vintage, 2016

\begin{tabular}{|c|c|c|c|c|c|c|c|c|c|c|c|c|c|c|}
\hline $\begin{array}{l}\text { Sex } \\
(\%)\end{array}$ & $<5$ & $5 \leq,<10$ & $10 \leq,<15$ & $15 \leq,<20$ & $20 \leq,<25$ & $25 \leq,<30$ & $30 \leq,<35$ & $35 \leq,<40$ & $40 \leq$ & Subtotal & Unspecified & Total & Mean age & $\overline{S D}$ \\
\hline Male & $\begin{array}{l}19,888 \\
(41.2)\end{array}$ & $\begin{array}{l}12,785 \\
(26.5)\end{array}$ & $\begin{array}{l}6845 \\
(14.2)\end{array}$ & $\begin{array}{l}3805 \\
(7.9)\end{array}$ & $\begin{array}{l}2284 \\
(4.7)\end{array}$ & $\begin{array}{l}1301 \\
(2.7)\end{array}$ & $\begin{array}{l}759 \\
(1.6)\end{array}$ & $\begin{array}{l}438 \\
(0.9)\end{array}$ & $\begin{array}{l}159 \\
(0.3)\end{array}$ & $48,264(100.0)$ & 27 & 48,291 & 8.33 & 7. \\
\hline Female & $\begin{array}{l}8816 \\
(33.3)\end{array}$ & $\begin{array}{l}6404 \\
(24.2)\end{array}$ & $\begin{array}{l}4102 \\
(15.5)\end{array}$ & $\begin{array}{l}2773 \\
(10.5)\end{array}$ & $\begin{array}{l}1872 \\
(7.1)\end{array}$ & $\begin{array}{l}1248 \\
(4.7)\end{array}$ & $\begin{array}{l}727 \\
(2.7)\end{array}$ & $\begin{array}{l}404 \\
(1.5)\end{array}$ & $\begin{array}{l}154 \\
(0.6)\end{array}$ & $26,500(100.0)$ & 8 & 26,508 & 10.4 & 9 \\
\hline Total & $\begin{array}{l}28,704 \\
(38.4)\end{array}$ & $\begin{array}{l}19,189 \\
(25.7)\end{array}$ & $\begin{array}{l}10,947 \\
(14.6)\end{array}$ & $\begin{array}{l}6578 \\
(8.8)\end{array}$ & $\begin{array}{l}4156 \\
(5.6)\end{array}$ & $\begin{array}{l}2549 \\
(3.4)\end{array}$ & $\begin{array}{l}1486 \\
(2.0)\end{array}$ & $\begin{array}{l}842 \\
(1.1)\end{array}$ & $\begin{array}{l}313 \\
(0.4)\end{array}$ & $74,764(100.0)$ & 35 & 74,799 & 9.07 & \\
\hline
\end{tabular}

Values in parentheses under each figure represent the percentage relative to the subtotal in each row. The above data were obtained from the patient survey Abbreviation: HDF hemodiafiltration

whereas the least common combination was off-line HDF/ pre-dilution (501 patients). The blood flow rate in on-line HDF tended to be higher than that in off-line HDF; however, there was no difference in blood flow rate between pre-dilution and post-dilution (Fig. 34, Additional file 31: Table S31). The on-line HDF/pre-dilution combination had the highest blood flow rate, with a mean of $229 \mathrm{~mL} / \mathrm{min}$; in this combination, $>50 \%$ of patients demonstrated a blood flow rate of $220 \mathrm{~mL} / \mathrm{min}$ or higher, whereas $9.1 \%$ of patients demonstrated a blood flow rate of $300 \mathrm{~mL} / \mathrm{min}$ or higher. On-line HDF was found to involve a longer dialysis time (Fig. 35, Additional file 32: Table S32).

The highest substitution volume was observed in the on-line HDF/pre-dilution combination (mean, 39.9 L), whereas the on-line HDF/post-dilution combination had a substitution volume of $10.2 \mathrm{~L}$ (Fig. 36, Additional file 33: Table S33). In off-line HDF, the substitution volume in pre-dilution and post-dilution was 12.0 and $8.0 \mathrm{~L}$, respectively. There were no changes in substitution volume or its distribution over time in on-line $\mathrm{HDF} /$ pre-dilution or on-line $\mathrm{HDF} /$ post-dilution. In contrast, the substitution volume for the off-line HDF/pre-dilution combination exhibited a slight upward trend yearly. The substitution volume for the off-line HDF/post-dilution has remained at around $8.0 \mathrm{~L}$.

\section{Comparison of HDF patients and in-center HD patients}

We compared the background factors and treatment indices of HDF patients with those of in-center HD patients by

Table 12 Prevalent dialysis patient distribution, by HDF/HD and primary disease, 2016

\begin{tabular}{lll}
\hline Primary disease & HDF (\%) & HD (\%) \\
\hline Diabetes & $26,768(35.8)$ & $93,965(40.2)$ \\
Chronic glomerulonephritis & $24,519(32.8)$ & $63,328(27.1)$ \\
Nephrosclerosis & $6443(8.6)$ & $24,053(10.3)$ \\
PKD & $2807(3.8)$ & $8319(3.6)$ \\
Chronic pyelonephritis & $687(0.9)$ & $2083(0.9)$ \\
Others & $13,575(18.1)$ & $41,956(18.0)$ \\
Total & $74,799(100.0)$ & $233,704(100.0)$
\end{tabular}

Values in parentheses under each figure represent the percentage relative to the subtotal in each row. The above data were obtained from the patient survey Abbreviations: $H D$ hemodialysis, HDF hemodiafiltration, PKD polycystic kidney disease using on-line versus off-line and by dilution methods (Table 13). HDF patients did not demonstrate evident sex differences but were younger and tended to have longer dialysis vintages than in-center HD patients. The prevalence of diabetic nephropathy as a primary disease among HDF patients was lower than that among in-center HD patients. The dialysis time was around $4 \mathrm{~h}$ for in-center HD patients and HDF patients in all HDF modalities. The blood flow rate in HDF patients was relatively high, which may be due to factors such as age differences between the patients. Although small-molecule clearance generally decreased at high rates of pre-dilution, we found no difference in single-pool $K t / V$ urea ( $K t / V$ sp) between HD and HDF or between dilution modalities. This could be due to the slightly higher values for dialysis time and blood flow rate for the on-line HDF/pre-dilution combination, which involves a large substitution volume, than for in-center HD.

As for nutritional status indices, there were no evident differences between HDF and HD in serum albumin levels or nPCR. Creatinine levels and \%CGR were higher with in on-line HDF/pre-dilution and post-dilution than in HD and roughly equivalent between HD and off-line HDF/pre-dilution and post-dilution. The CRP levels tended to be lower for on-line HDF/pre-dilution and post-dilution than in HD.

As treatment indicators for chronic kidney diseasemineral and bone disorder, the corrected calcium levels and intact PTH levels did not differ between modalities. Phosphorus levels were high with on-line HDF/pre-dilution and post-dilution. As for total cholesterol levels and hemoglobin levels, there were no evident differences between treatment modalities.

The differences in clinical indices between HDF patients and in-center HD patients may be due to selection bias such as patient age.

\section{Chapter 5: peritoneal dialysis (PD) $P D$ patient dynamics}

According to the facility survey, there were $9021 \mathrm{PD}$ patients in 2016. The variations of PD therapy were PD only and combination with HD once weekly, twice weekly, thrice weekly, and other combinations; the number of patients in each modality was 7190, 1560, 169, 38, 


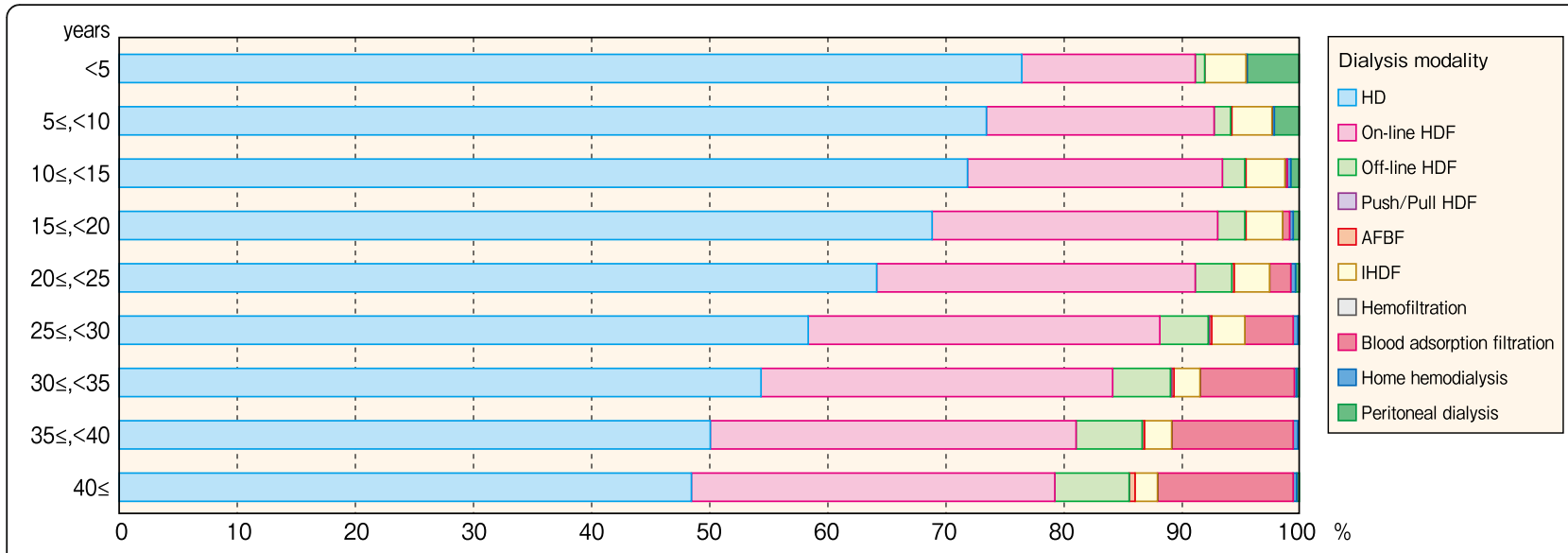

Fig. 33 Prevalent dialysis patient distribution, by dialysis modality and dialysis vintage, 2016. Abbreviations: AFBF acetate-free biofiltration, HD hemodialysis, HDF hemodiafiltration, IHDF intermittent infusion hemodiafiltration

and 64, respectively (Table 1). Although the total number of PD patients has been gradually decreasing since 2009, it must be noted that because the JRDR survey mainly targets facilities performing HD, PD patients treated at other facilities may not be included (Fig. 37, Additional file 34: Table S34). In 2015, we began to investigate the number of incident PD patients, which decreased from 2197 in 2015 to 1946 in 2016.

There were 8693 patients undergoing PD (including in combination with HD) in the patient survey; these patients consisted of 5655 (65.1\%) men and 3038 (34.9\%) women with mean ages of 62.96 and 63.41 years, respectively (Table 14). The age distribution peaked at 60 74 years, which was the same trend as with HD patients. Of all patients currently undergoing PD, $44.2 \%$ had vintages of $<2$ years whereas $7.1 \%$ had vintages of 8 years or longer (Table 15). Diabetic nephropathy was the primary disease in $31.9 \%$ of PD patients, which was nearly equal to the $31.0 \%$ PD patients with chronic glomerulonephritis as the primary disease (Table 16). The percentage of diabetic nephropathy in PD patients was lower than that in HD patients.

\section{$P D+H D$ combined therapy}

Of the 8693 PD patients for whom responses were provided in the 2016 patient survey, the number of patients undergoing PD alone was 6931 (79.7\%), and this number has remained virtually unchanged since 2009 (Fig. 38, Additional file 35: Table S35). Only 3.4\% of patients with PD vintages $<2$ years were undergoing combined therapy with $\mathrm{HD}(\mathrm{F})$; however, the percentage of combined therapy increased as the PD vintages lengthened, increasing to $53.1 \%$ of patients with PD vintages of at least 8 years (Fig. 39, Additional file 36: Table S36). Most patients undergoing combined therapy did so once a week as $84.9 \%$ of all combined therapy patients.

\section{PD prescriptions}

PD prescriptions were examined for the 6931 patients undergoing PD alone according to the patient survey. Of these 6931 patients, responses about the daily PD fluid volume were obtained for 4476 patients (64.6\%). The mean PD fluid volume for men and women was 6.59 and $5.69 \mathrm{~L}$, respectively. The PD fluid volume decreased as age increased (Fig. 40, Additional file 37:

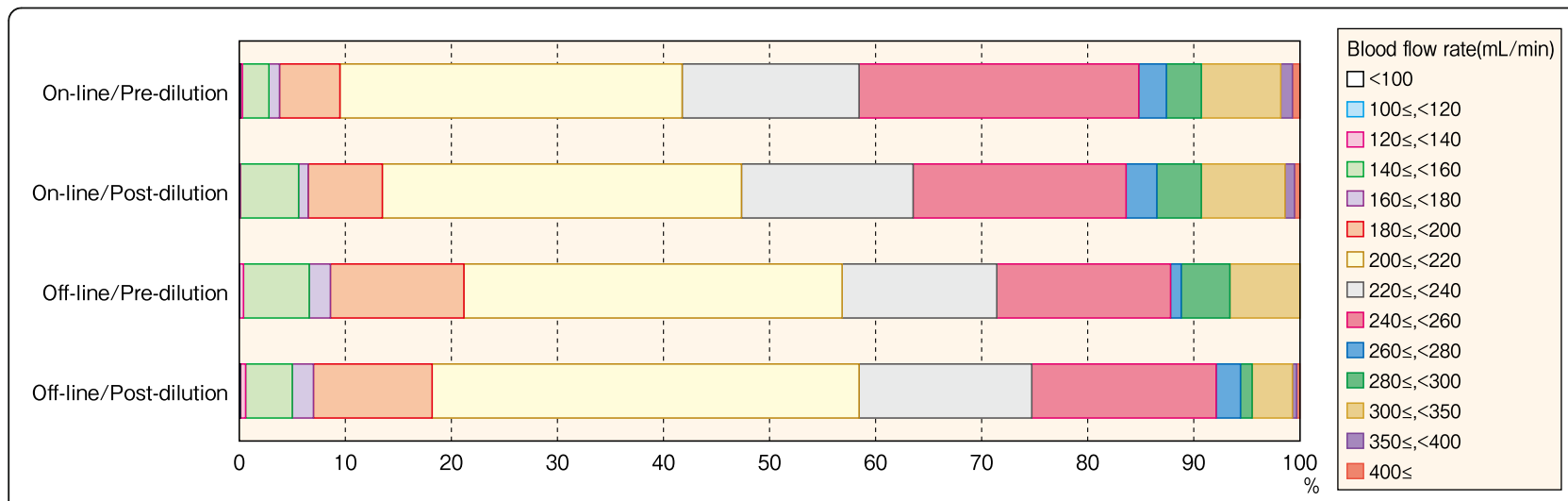

Fig. 34 HDF patient distribution, by dilution mode and blood flow rate, 2016. Abbreviation: HDF hemodiafiltration 


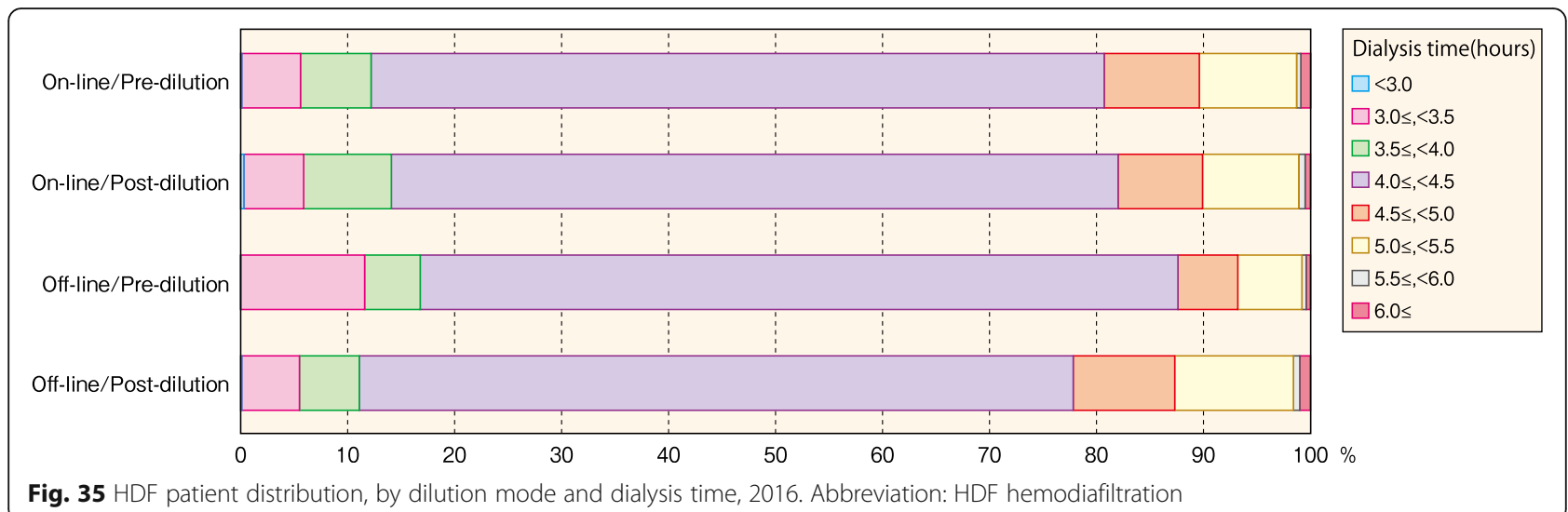

Table S37) and increased as PD vintages lengthened (Fig. 41, Additional file 38: Table S38). The daily PD treatment time was stated for 4393 patients (63.4\%); there was no evident difference based on sex (men $18.0 \mathrm{~h}$, women $17.7 \mathrm{~h}$ ) or age (Fig. 42, Additional file 39: Table S39). In contrast, longer PD vintages were correlated with longer PD treatment times; $74.9 \%$ of all patients with a PD vintage of 8 years or longer had a daily PD treatment time of $24 \mathrm{~h}$ (Fig. 43, Additional file 40: Table S40).

Responses about the use of APD were received for 4672 patients $(67.4 \%), 41.9 \%$ of whom had undergone APD (Table 17). Responses about changing the maneuver of PD fluid were received for 4640 patients (66.9\%); UV sterilization connecting devices, completely manual methods, and thermal sterilization connecting devices were used for 53.4, 27.1, and $16.7 \%$ of these patients, respectively (Table 18).

\section{Residual kidney function (urine volume and residual kidney $K t / V)$}

Residual kidney function was evaluated by residual kidney $K t / V$ and urine volume for the 6931 patients undergoing PD alone. Mean urine volume responses were obtained for 3825 patients (55.2\%), which represents a
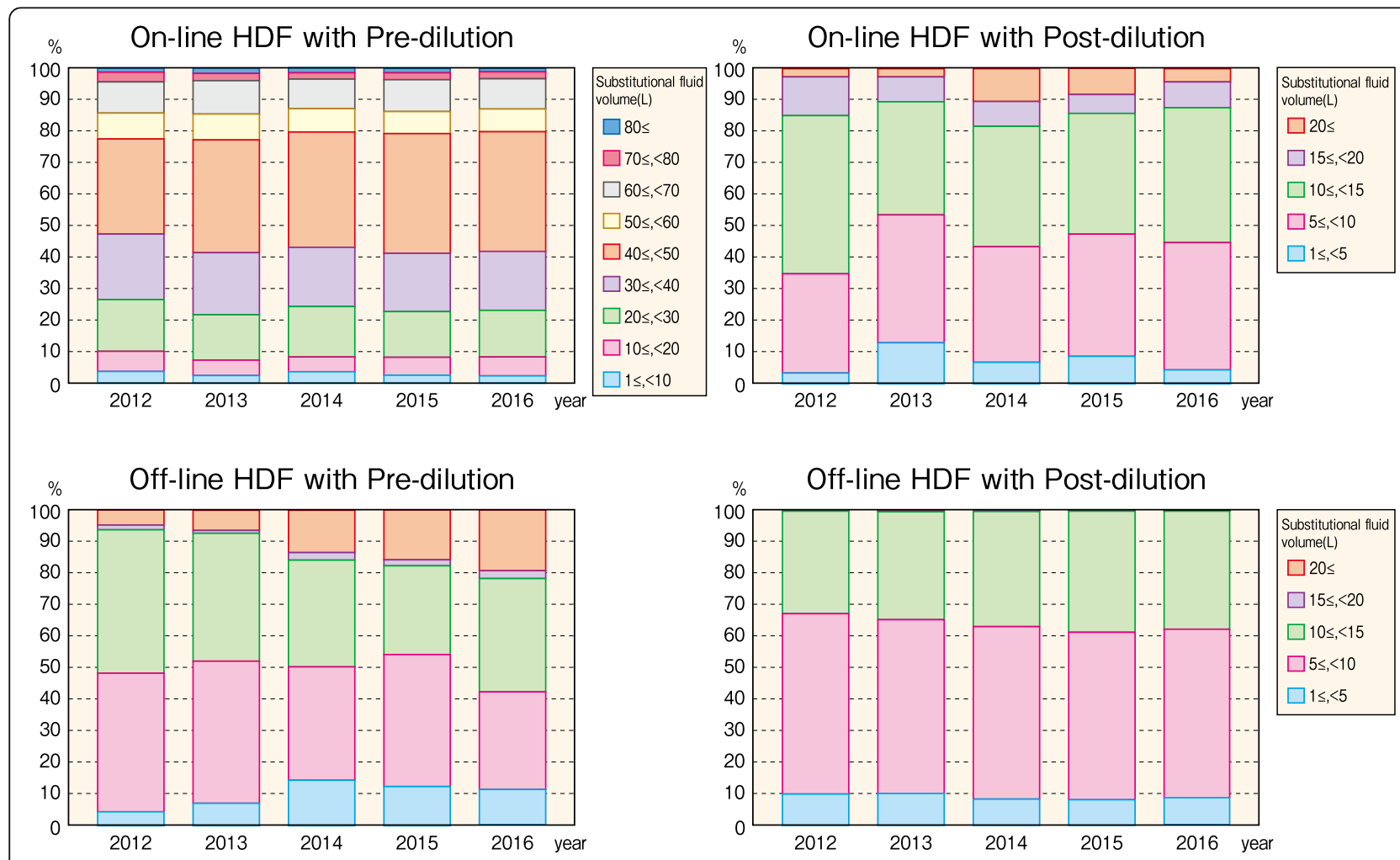

Fig. 36 Substitution fluid volume, by HDF modality and dilution mode, 2012-2016. Abbreviation: HDF hemodiafiltration 
Table 13 Comparisons of HD, on-line HDF, and off-line HDF, 2016

\begin{tabular}{|c|c|c|c|c|c|}
\hline & Hemodialysis & On-line HDF & & Off-line HDF & \\
\hline & & Pre-dilution & Post-dilution & Pre-dilution & Post-dilution \\
\hline Number of patients (\%) & 209,536 & 48,457 & 2227 & 501 & 3250 \\
\hline Male & 135,450 & 31,658 & 1454 & 319 & 1970 \\
\hline Male (\%) & 64.6 & 65.3 & 65.3 & 63.7 & 60.6 \\
\hline Percentage of diabetes (\%) & 40.3 & 35.2 & 32.9 & 32.9 & 32.0 \\
\hline Age & $68.93 \pm 12.26$ & $65.44 \pm 12.44$ & $64.77 \pm 12.65$ & $66.46 \pm 12.11$ & $66.66 \pm 11.89$ \\
\hline Dialysis vintage (years) & $6.94 \pm 7.14$ & $9.18 \pm 8.41$ & $10.51 \pm 9.07$ & $11.24 \pm 9.42$ & $12.56 \pm 10.07$ \\
\hline Dialysis time (minute) & $238.81 \pm 32.04$ & $245.21 \pm 28.96$ & $243.78 \pm 29.23$ & $238.43 \pm 28.9$ & $247.11 \pm 30.37$ \\
\hline Blood flow rate $(\mathrm{mL} / \mathrm{min})$ & $206.0 \pm 35.5$ & $228.7 \pm 40.0$ & $224.9 \pm 42.2$ & $215.8 \pm 38.4$ & $214.3 \pm 36.2$ \\
\hline$K t /$ sp male* & $1.42 \pm 0.26$ & $1.45 \pm 0.26$ & $1.46 \pm 0.28$ & $1.41 \pm 0.26$ & $1.46 \pm 0.27$ \\
\hline$K t / V s p$ female* & $1.64 \pm 0.31$ & $1.70 \pm 0.32$ & $1.73 \pm 0.35$ & $1.62 \pm 0.32$ & $1.71 \pm 0.33$ \\
\hline Serum albumin (male) & $3.57 \pm 0.45$ & $3.63 \pm 0.38$ & $3.61 \pm 0.36$ & $3.57 \pm 0.46$ & $3.55 \pm 0.45$ \\
\hline Serum albumin (female) & $3.50 \pm 0.44$ & $3.58 \pm 0.37$ & $3.55 \pm 0.38$ & $3.56 \pm 0.41$ & $3.49 \pm 0.43$ \\
\hline Normalized protein catabolic rate $(\mathrm{g} / \mathrm{kg} / \mathrm{day}, \mathrm{male})^{*}$ & $0.84 \pm 0.17$ & $0.86 \pm 0.16$ & $0.87 \pm 0.16$ & $0.86 \pm 0.17$ & $0.85 \pm 0.17$ \\
\hline Normalized protein catabolic rate (g/kg/day, female)* & $0.87 \pm 0.18$ & $0.90 \pm 0.18$ & $0.90 \pm 0.18$ & $0.92 \pm 0.22$ & $0.89 \pm 0.18$ \\
\hline Pre-dialysis serum creatinine (male)* & $10.81 \pm 2.78$ & $11.41 \pm 2.71$ & $11.58 \pm 2.83$ & $10.99 \pm 2.64$ & $10.88 \pm 2.72$ \\
\hline Pre-dialysis serum creatinine (female)* & $8.96 \pm 2.34$ & $9.55 \pm 2.16$ & $9.46 \pm 2.04$ & $9.18 \pm 1.90$ & $9.08 \pm 2.10$ \\
\hline Percent creatinine generation rate (\%CGR) (male)* & $98.77 \pm 25.56$ & $102.10 \pm 23.81$ & $102.44 \pm 22.79$ & $97.10 \pm 24.91$ & $98.17 \pm 24.60$ \\
\hline Percent creatinine generation rate (\%CGR) (female) ${ }^{*}$ & $97.93 \pm 26.36$ & $102.60 \pm 23.76$ & $102.28 \pm 23.69$ & $98.17 \pm 25.96$ & $98.60 \pm 24.85$ \\
\hline Serum CRP level (mg/dL) & $0.66 \pm 1.88$ & $0.52 \pm 1.41$ & $0.51 \pm 1.30$ & $0.69 \pm 1.84$ & $0.82 \pm 2.23$ \\
\hline Pre-dialysis serum calcium (mg/dL) & $9.16 \pm 0.75$ & $9.14 \pm 0.72$ & $9.21 \pm 0.70$ & $9.22 \pm 0.77$ & $9.27 \pm 0.80$ \\
\hline Pre-dialysis serum phosphorus (mg/dL) & $5.17 \pm 1.44$ & $5.35 \pm 1.42$ & $5.41 \pm 1.39$ & $5.28 \pm 1.49$ & $5.17 \pm 1.46$ \\
\hline Intact PTH level (pg/ml) & $176.5 \pm 170.1$ & $181.6 \pm 168.0$ & $180.52 \pm 181.9$ & $182.5 \pm 201.7$ & $170.7 \pm 189.0$ \\
\hline Pre-dialysis serum total cholesterol (mg/dl) & $155.4 \pm 36.1$ & $159.1 \pm 35.7$ & $162.3 \pm 36.8$ & $154.7 \pm 35.7$ & $155.0 \pm 36.4$ \\
\hline Pre-dialysis hemoglobin (g/dL) & $10.77 \pm 1.31$ & $10.95 \pm 1.23$ & $10.95 \pm 1.21$ & $10.86 \pm 1.36$ & $10.77 \pm 1.31$ \\
\hline
\end{tabular}

Population: in-center hemodialysis or hemodiafiltration (three times a week). The above data were obtained from the patient survey. For the indices from age to percent creatinine generation rate (female), "mean \pm SD" are shown. Note: Total number of each index was different from each other because response rate for the question was different in each other

Abbreviations: $K t / N s p$ index for standardized dialysis dose defined as $K$ urea clearance, $t$ dialysis time, $V$ body fluid volume; $s p$ single pool; $P T H$ parathyroid hormone

${ }^{*} \mathrm{Kt} / \mathrm{V}, \mathrm{nPCR}$, creatinine concentration, and \%CGR were summarized in the patients with vintages of 2 years or more and receiving dialysis three times a week

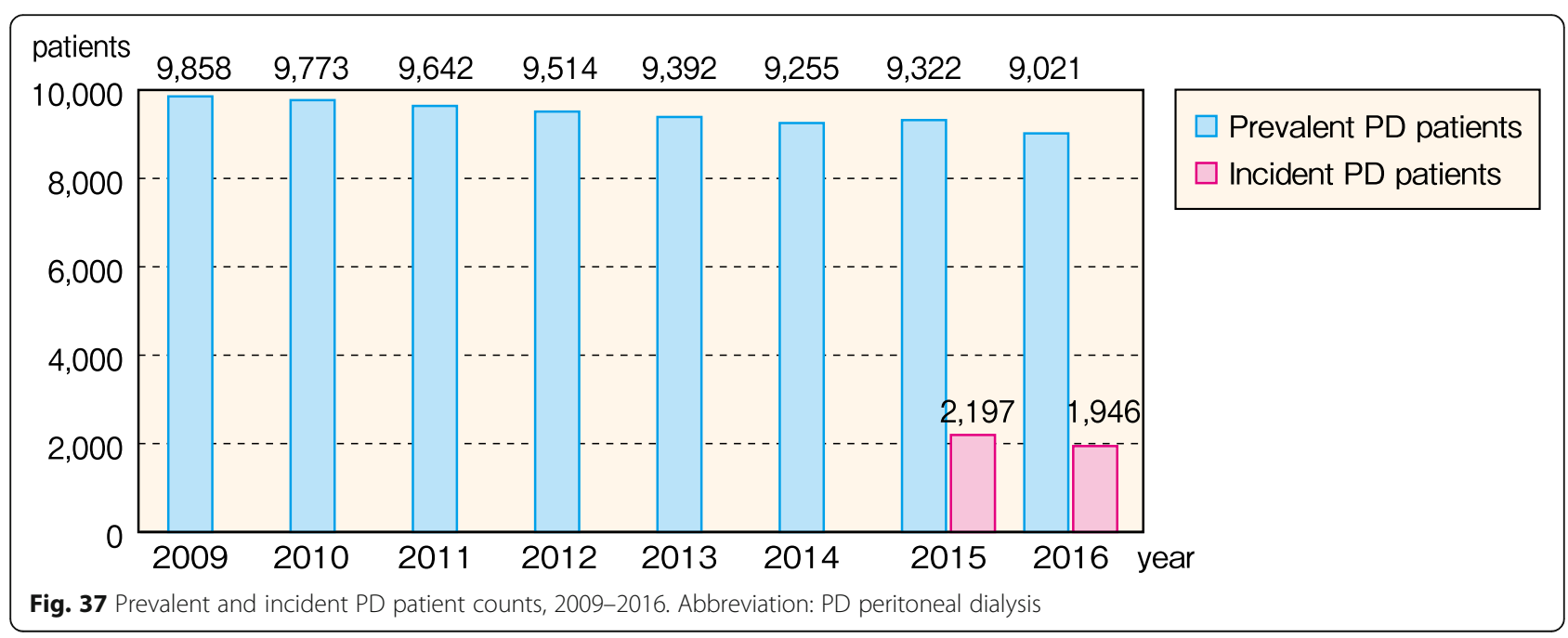


Table 14 Prevalent PD patient distribution, by age and sex, 2016

\begin{tabular}{lllll}
\hline Age & Male & Female & Subtotal & Total \\
\hline$<15$ & $41(0.7)$ & $44(1.4)$ & $85(1.0)$ & $85(1.0)$ \\
$15 \leq,<30$ & $58(1.0)$ & $37(1.2)$ & $95(1.1)$ & $95(1.1)$ \\
$30 \leq,<45$ & $392(6.9)$ & $224(7.4)$ & $616(7.1)$ & $616(7.1)$ \\
$45 \leq,<60$ & $1518(26.8)$ & $788(25.9)$ & $2306(26.5)$ & $2306(26.5)$ \\
$60 \leq,<75$ & $2558(45.2)$ & $1204(39.6)$ & $3762(43.3)$ & $3762(43.3)$ \\
$75 \leq,<90$ & $1028(18.2)$ & $675(22.2)$ & $1703(19.6)$ & $1703(19.6)$ \\
$90 \leq$ & $60(1.1)$ & $66(2.2)$ & $126(1.4)$ & $126(1.4)$ \\
Total & $5655(100.0)$ & $3038(100.0)$ & $8693(100.0)$ & $8693(100.0)$ \\
Mean & 62.96 & 63.41 & 63.12 & 63.12 \\
SD & 13.78 & 15.65 & 14.46 & 14.46 \\
\hline
\end{tabular}

Values in parentheses under each figure represent the percentage relative to the total in each column. The above data were obtained from the patient survey Abbreviation: $P D$ peritoneal dialysis

slightly lower response rate than those for the other questions about PD discussed above. The mean urine volume for men and women was 778 and $665 \mathrm{~mL}$, respectively (Fig. 44, Additional file 41: Table S41). There were no evident differences by age, but urine volume decreased as PD vintage increased; $60.0 \%$ of patients with a PD vintage of 8 years or longer had a urine volume of $<$ $100 \mathrm{~mL}$ (Fig. 45, Additional file 42: Table S42).

Residual kidney $K t / V$ responses were obtained for 2070 patients; the mean was 0.65 for men and 0.61 for women (Fig. 46, Additional file 43: Table S43). There were no evident differences by age, but residual kidney $K t / V$ showed a downward trend as the PD vintage lengthened (Fig. 47, Additional file 44: Table S44); 83.1\% of patients with a PD vintage of 8 years or longer had a residual renal $K t / V$ of $<0.4$.
Table 16 Prevalent patient distribution, by PD or HD and primary disease, 2016

\begin{tabular}{|c|c|c|}
\hline Primary disease & Peritoneal dialysis (\%) & Hemodialysis (\%) \\
\hline Diabetes & $2772(31.9)$ & $93,905(40.2)$ \\
\hline Chronic glomerulonephritis & $2695(31.0)$ & $63,287(27.1)$ \\
\hline Nephrosclerosis & $1110(12.8)$ & $24,045(10.3)$ \\
\hline PKD & $274(3.2)$ & 8318 (3.6) \\
\hline Chronic pyelonephritis & $99(1.1)$ & $2081(0.9)$ \\
\hline Others & $1743(20.1)$ & $41,930(18.0)$ \\
\hline Total & $8693(100.0)$ & $233,566(100.0)$ \\
\hline
\end{tabular}

\section{$P D$ dose (ultrafiltration volume and PD Kt/V)}

The PD dose was evaluated by ultrafiltration volume and PD $K t / V$ for 6931 patients undergoing PD alone. Mean ultrafiltration volume responses were obtained for 4383 patients (63.2\%). The mean ultrafiltration volume for men and women was 632 and $582 \mathrm{~mL}$, respectively (Fig. 48, Additional file 45: Table S45). There were no evident differences by age, but ultrafiltration volume showed an upward trend as the PD vintage lengthened; $55.6 \%$ of patients with a PD vintage of 8 years or longer had an ultrafiltration volume of $800 \mathrm{~mL}$ or more (Fig. 49, Additional file 46: Table S46).

Mean PD $K t / V$ responses were obtained for 2238 patients (32.3\%). The mean PD $K t / V$ for men and women was 1.17 and 1.32, respectively; thus, women tended to have higher values, but there were no evident tendencies by age (Fig. 50, Additional file 47: Table S47). PD $K t / V$ also showed an upward trend as PD vintage lengthened; $63.8 \%$ of patients with a PD vintage of 8 years or longer had a PD $K t / V$ of 1.6 or more (Fig. 51, Additional file 48: Table S48).

Table 15 Prevalent PD patient distribution, by PD vintage and sex, 2016

\begin{tabular}{lllll}
\hline PD vintage & Male & Female & Subtotal & Total \\
\hline$<1$ & $885(23.8)$ & $428(21.6)$ & $1313(23.0)$ & $1313(23.0)$ \\
$1 \leq,<2$ & $821(22.1)$ & $389(19.6)$ & $1210(21.2)$ & $1210(21.2)$ \\
$2 \leq,<4$ & $999(26.8)$ & $530(26.7)$ & $1529(26.8)$ & $1529(26.8)$ \\
$4 \leq,<6$ & $545(14.6)$ & $287(14.5)$ & $832(14.6)$ & $832(14.6)$ \\
$6 \leq,<8$ & $244(6.6)$ & $172(8.7)$ & $416(7.3)$ & $416(7.3)$ \\
$8 \leq,<10$ & $92(2.5)$ & $90(4.5)$ & $182(3.2)$ & $182(3.2)$ \\
$10 \leq$ & $137(3.7)$ & $88(4.4)$ & $225(3.9)$ & $225(3.9)$ \\
Subtotal & $3723(100.0)$ & $1984(100.0)$ & $5707(100.0)$ & $5707(100.0)$ \\
No information available & 1932 & 1054 & 2986 & 2986 \\
Total & 5655 & 3038 & 8693 & 8693 \\
Mean & 3.04 & 3.46 & 3.19 & 3.19 \\
SD & 2.96 & 3.35 & 3.11 & 3.11 \\
\hline Val & & & \\
\hline
\end{tabular}

Values in parentheses under each figure represent the percentage relative to the total in each column. The above data were obtained from the patient survey Abbreviation: $P D$ peritoneal dialysis 


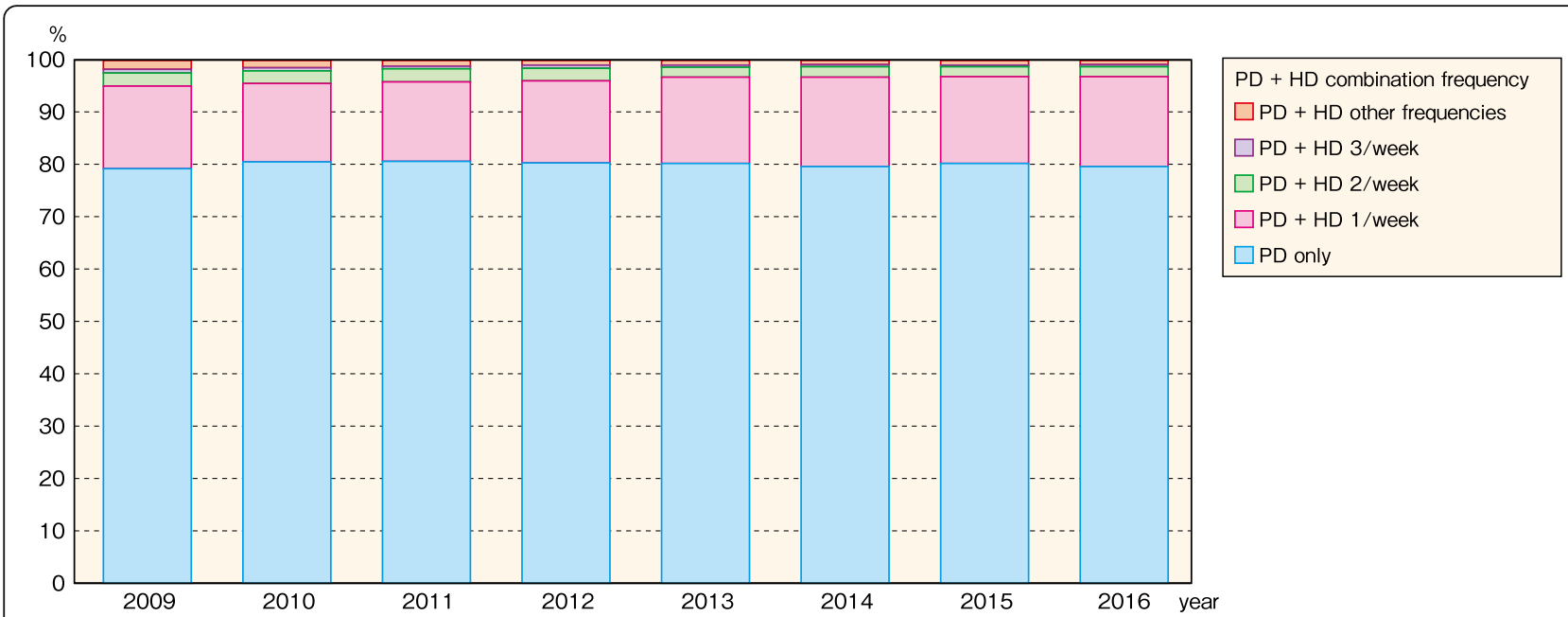

Fig. 38 Prevalent patient distribution, by PD + HD combination frequency, 2009-2016. Abbreviations: HD hemodialysis, PD peritoneal dialysis

\section{PET and D/P Cr ratio}

The JSDT PD guideline recommends evaluating peritoneal function with peritoneal equilibration test (PET) once or twice a year [17]. The JRDR investigated "with or without" of PET and the dialysate/peritoneal creatinine (D/P $\mathrm{Cr}$ ) ratio. Among patients undergoing PD alone, a PET was performed for $45.9 \%$ of patients, whereas only fast $\mathrm{PET}^{1}$ was performed for $18.1 \%$ of patients, and no PET was performed for $36.0 \%$ of patients (Table 19). The mean $\mathrm{D} / \mathrm{P} \mathrm{Cr}$ ratio for men and women was 0.67 and 0.65 , respectively; thus, there was no sex-based difference, but an upward trend was observed as the age increased (Fig. 52, Additional file 49: Table S49). No consistent trend was seen in the $\mathrm{D} / \mathrm{P}$ Cr ratio by $\mathrm{PD}$ vintage, but the ratio decreased slightly to 0.63 among patients with a PD vintage of 8 years or longer (Fig. 53, Additional file 50: Table S50). The D/P Cr ratio by primary disease was high, 0.68 , for both diabetic nephropathy and nephrosclerosis (Fig. 54, Additional file 51: Table S51).

\section{Peritonitis and exit-site infections}

Peritonitis and catheter exit-site infections (ESIs) are major complications of PD and are therefore key factors that reduce PD continuation rates. The International Society for Peritoneal Dialysis (ISPD) guideline recommends monitoring the incidence of peritonitis as part of a continuous quality improvement program [18]. Peritonitis is defined as a white blood cell count of $100 / \mu \mathrm{L}$ (at least 50\% neutrophils) in PD drainage fluid; the

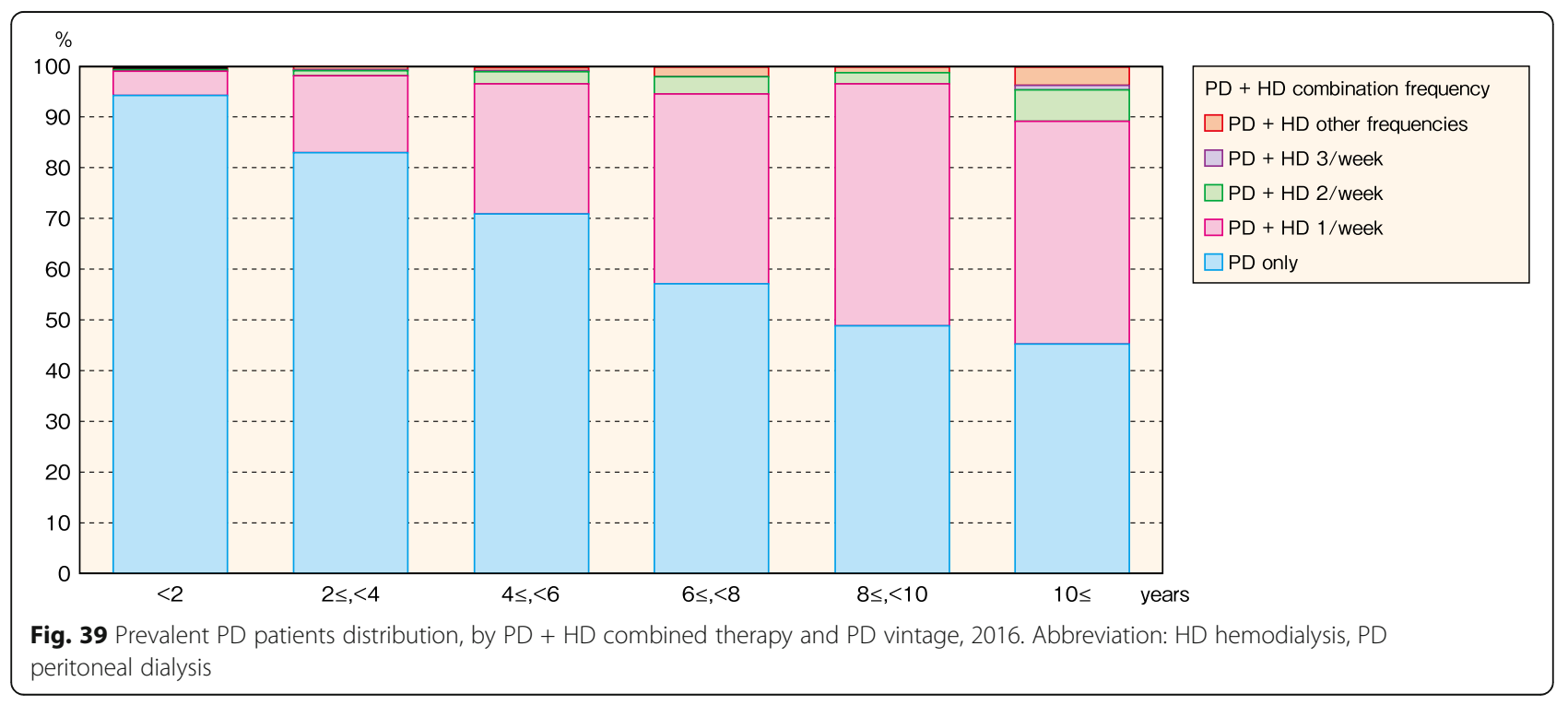




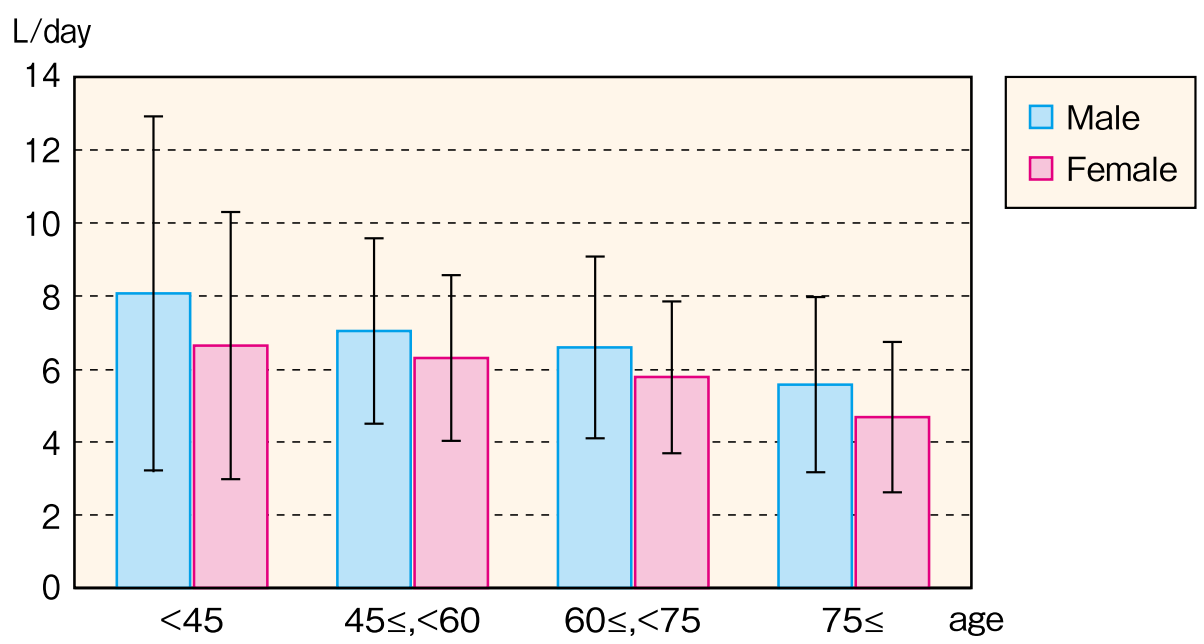

Fig. 40 PD fluid volume, by age and sex, 2016. Abbreviation: PD peritoneal dialysis

peritonitis rate per patient-year was calculated based on the ISPD guideline using the formula below. ESIs were defined as evident drainage of pus.

$$
\begin{aligned}
\text { Peritonitis rate }= & \text { peritonitis episodes in } 2016 \\
& \div \text { total months in } 2016 \text { on PD/12. }
\end{aligned}
$$

Of the 6931 patients undergoing PD alone, responses about peritonitis episodes were obtained from 4406 (63.6\%); peritonitis was observed at least once during the year in $14.5 \%$ of patients (Fig. 55, Additional file 52: Table S52). Among the 4406 patients for whom responses were obtained, the peritonitis rate was 0.24 per patient-year, which was the same as in 2015. Men had a rate of $0.26 \mathrm{ep-}$ isodes/patient-year, whereas women had a rate of $0.22 \mathrm{epi-}$ sodes/patient-year; thus, the peritonitis rate was slightly higher among men (Fig. 56, Additional file 53: Table S53). Patients younger than 45 years had a peritonitis rate of 0.21 episodes/patient-year, versus a rate of 0.30 episodes/ patient-year among patients aged 75 years and older; thus, the peritonitis rate tended to increase with age (Fig. 57, Additional file 54: Table S54). No consistent trend was found between peritonitis onset and PD vintage (Fig. 58, Additional file 55: Table S55). In addition, there was no evident difference in peritonitis rate by primary disease (Fig. 59, Additional file 56: Table S56).

Responses about ESIs were obtained from 4391 patients (63.4\%), 19.7\% of whom had at least one ESI during the year (Fig. 60, Additional file 57: Table S57).

\section{History of EPS}

Encapsulating peritoneal sclerosis (EPS) is a serious complication of PD; responses about a history of EPS and details of treatment were obtained for 13,025 patients who are currently undergoing PD or have a history of PD. We found that $696(5.3 \%)$ of these patients had a history of EPS; of these patients, $83.6 \%$ had a history of steroid use and $77.7 \%$ had a history of ablative surgery (Table 20). Of these 696 patients with a history of EPS, there were 418 men (60.1\%) and 278 women

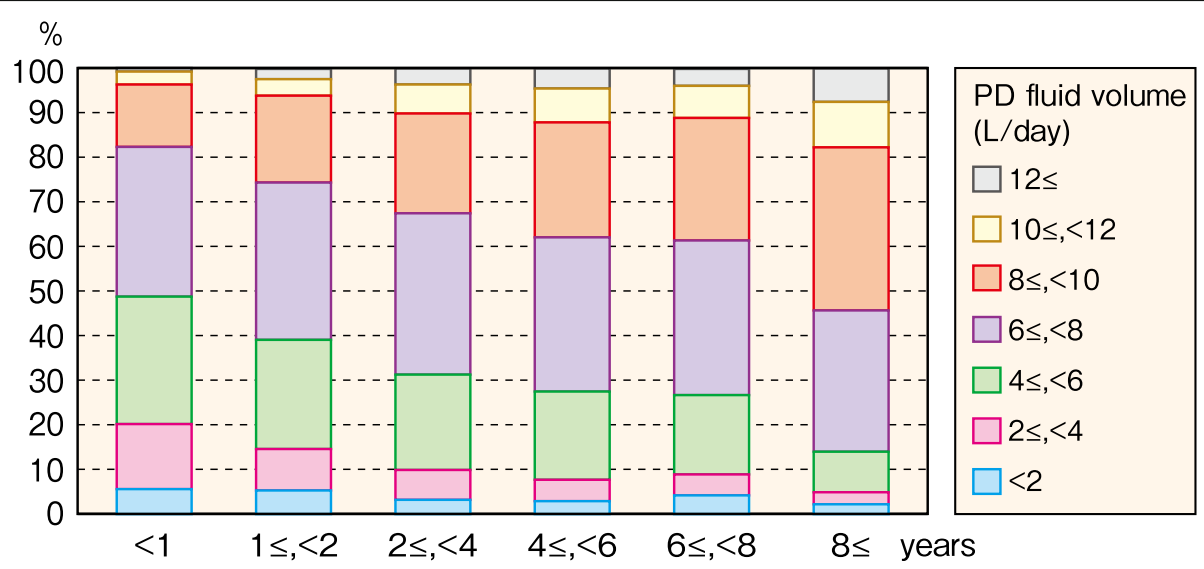

Fig. 41 PD fluid volume distribution, by PD vintage, 2016. Abbreviation: PD peritoneal dialysis 


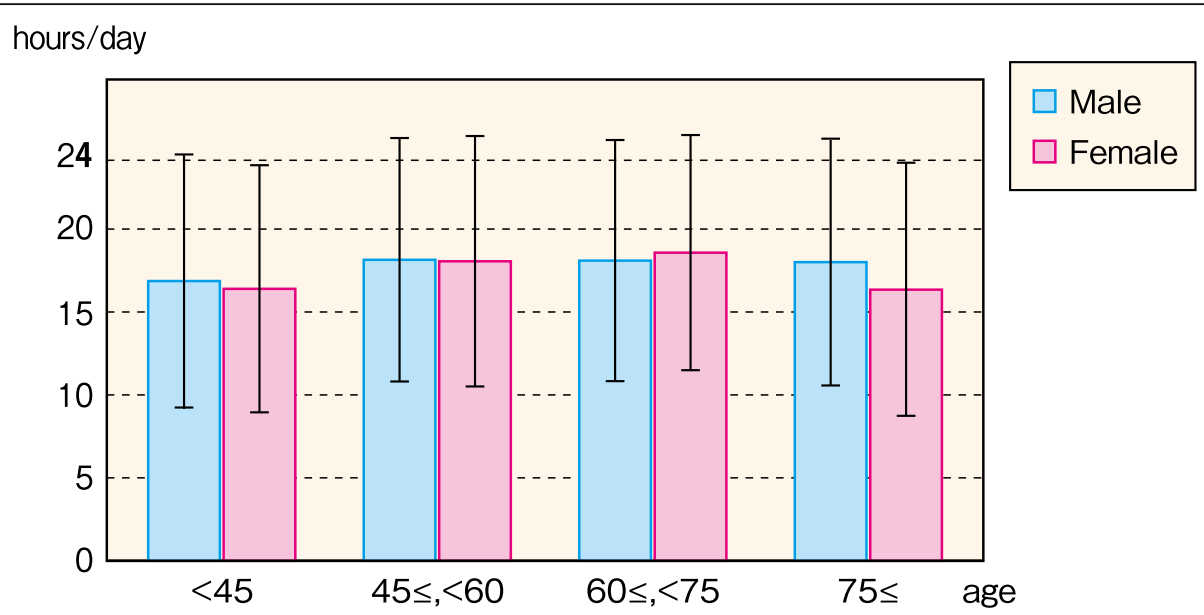

Fig. 42 PD treatment time, by age and sex, 2016. Abbreviation: PD peritoneal dialysis

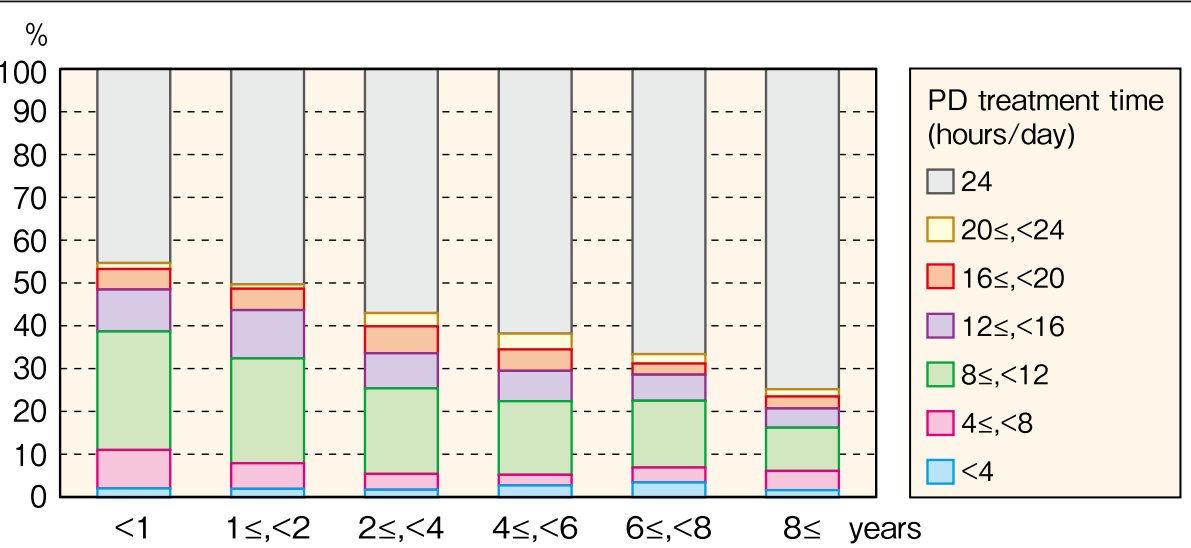

Fig. 43 PD treatment time distribution, by PD vintage, 2016. Abbreviation: PD peritoneal dialysis

Table 17 Prevalent PD patient counts, by APD machine use, 2016

\begin{tabular}{lcclcrl}
\hline & Patients on APD & Patients on CAPD & Subtotal & Unspecified & No information available & Total \\
\hline Patients (\%) & $1957(41.9)$ & $2715(58.1)$ & $4672(100.0)$ & 9 & 2250 & 6931
\end{tabular}

Values in parentheses under each figure represent the percentage relative to the subtotal in each row. The above data were obtained from the patient survey. Population: PD patients excluding PD + HD combination

Abbreviations: $A P D$ automated peritoneal dialysis, CAPD continuous peritoneal dialysis, $H D$ hemodialysis, $P D$ peritoneal dialysis 
Table 18 Prevalent PD patient counts, by PD fluid changing maneuver, 2016

\begin{tabular}{|c|c|c|c|c|c|c|c|c|}
\hline & $\begin{array}{l}\text { Manual } \\
\text { exchange }\end{array}$ & $\begin{array}{l}\text { Devices using UV } \\
\text { irradiation }\end{array}$ & $\begin{array}{l}\text { Devices using heat } \\
\text { sterilization }\end{array}$ & $\begin{array}{l}\text { Other devices including } \\
\text { semi-automated }\end{array}$ & Subtotal & Unspecified & $\begin{array}{l}\text { No information } \\
\text { available }\end{array}$ & Total \\
\hline $\begin{array}{l}\text { Patients } \\
\text { (\%) }\end{array}$ & $1258(27.1)$ & 2478 (53.4) & 775 (16.7) & $129(2.8)$ & $4640(100.0)$ & 19 & 2272 & 6931 \\
\hline
\end{tabular}

Values in parentheses under each figure represent the percentage relative to the subtotal in each row. The above data were obtained from the patient survey.

Population: PD patients excluding PD + HD combination

Abbreviations: $H D$ hemodialysis, $P D$ peritoneal dialysis
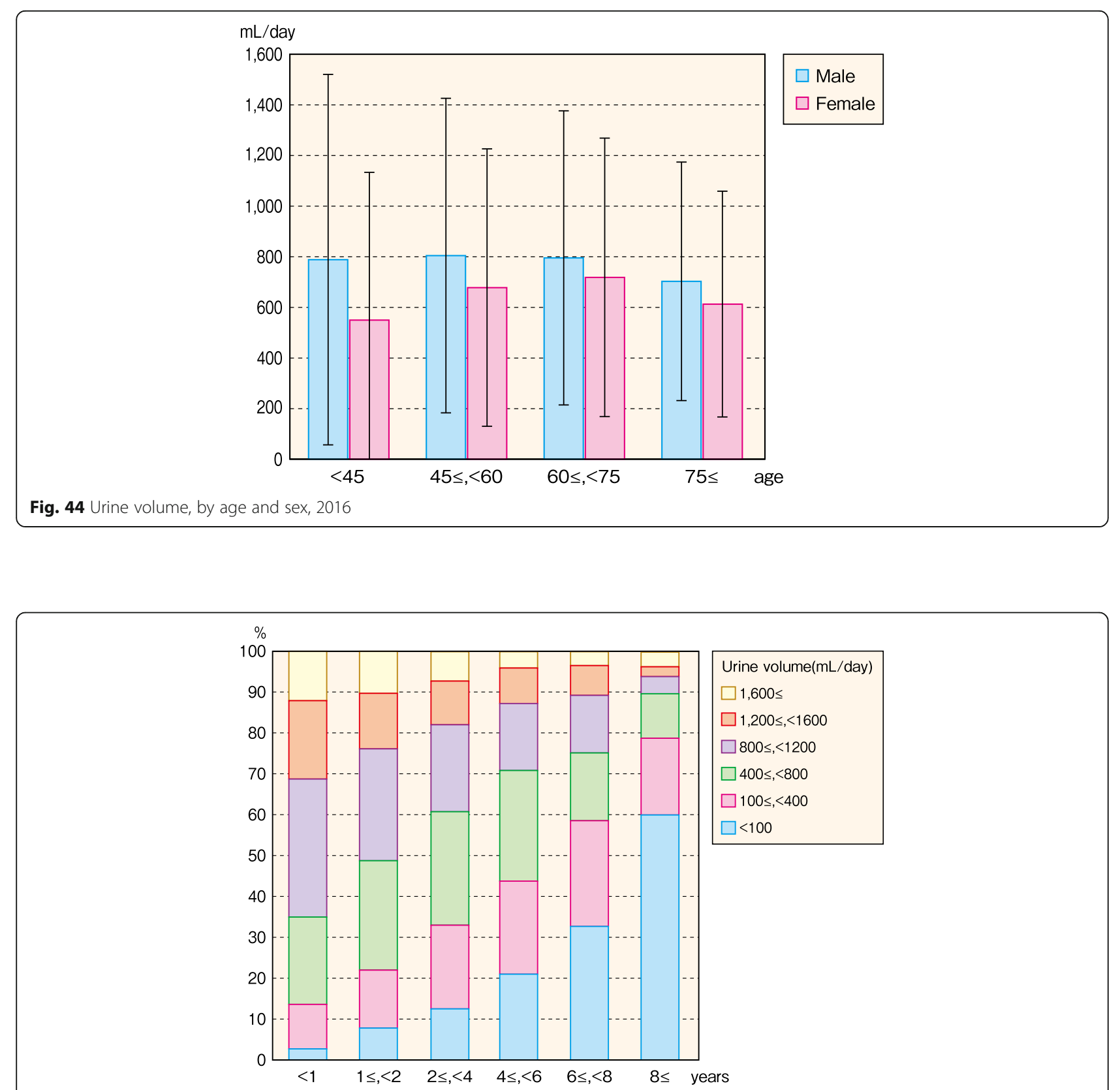

Fig. 45 Urine volume distribution, by PD vintage, 2016. Abbreviation: PD peritoneal dialysis 


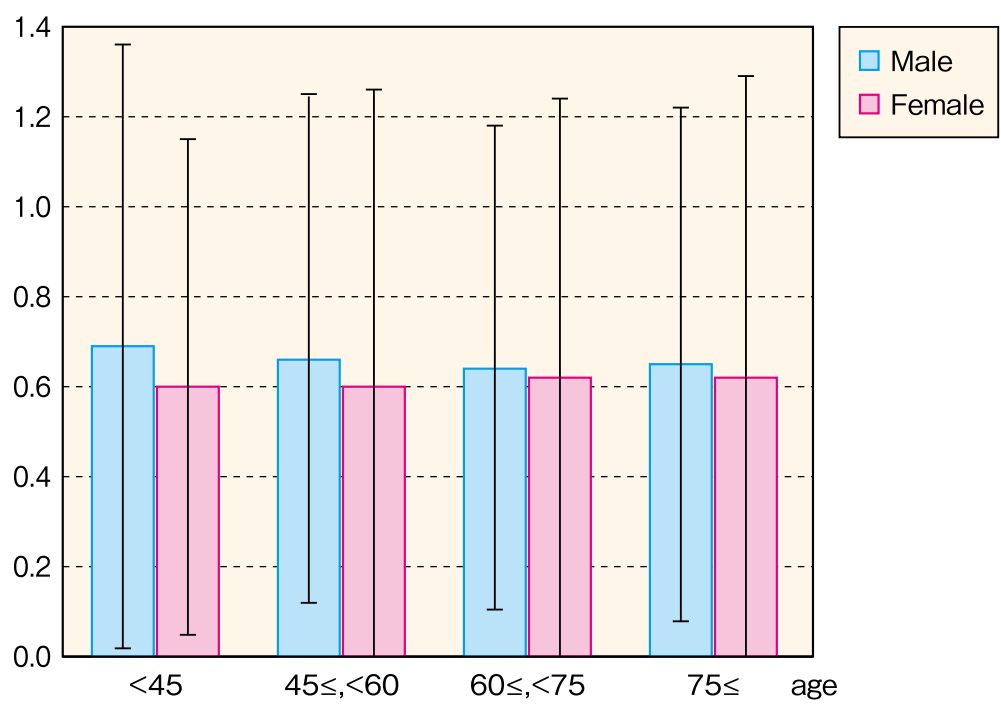

Fig. 46 Residual kidney $K t / V$, by age and sex, 2016. Abbreviations: $K t / V$ index for standardized dialysis dose defined as $K$ urea clearance, $t$ dialysis time, $V$ body fluid volume

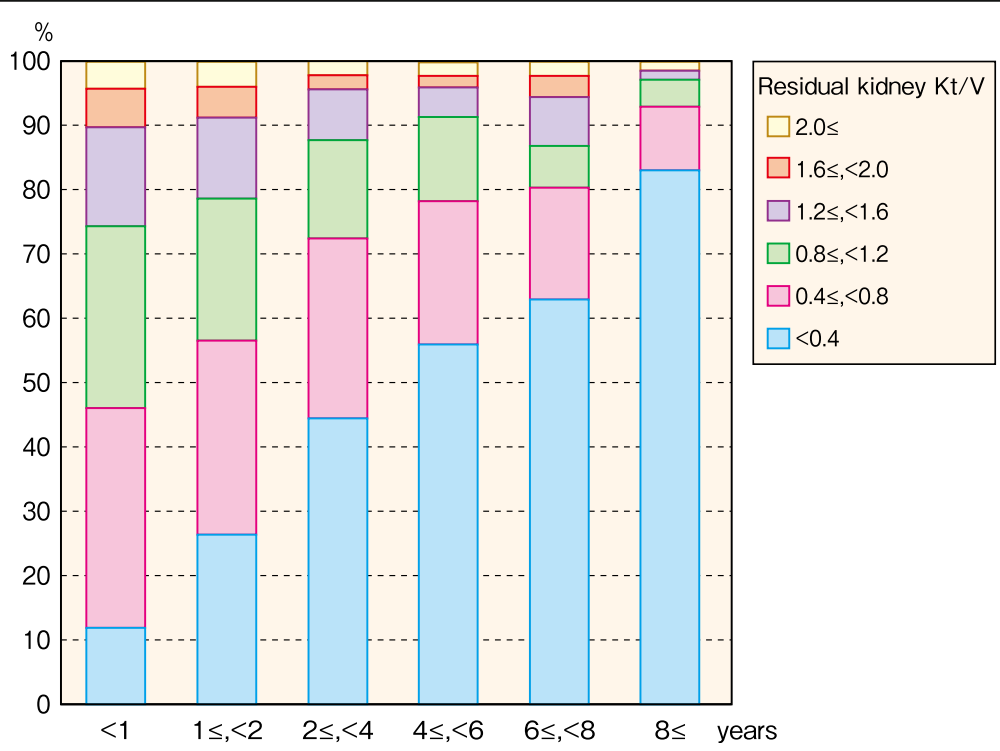

Fig. 47 Residual kidney $K t / V$ distribution, by PD vintage, 2016. Abbreviations: $K t / V$ index for standardized dialysis dose defined as $K$ urea clearance, $t$ dialysis time, $V$ body fluid volume; PD peritoneal dialysis 


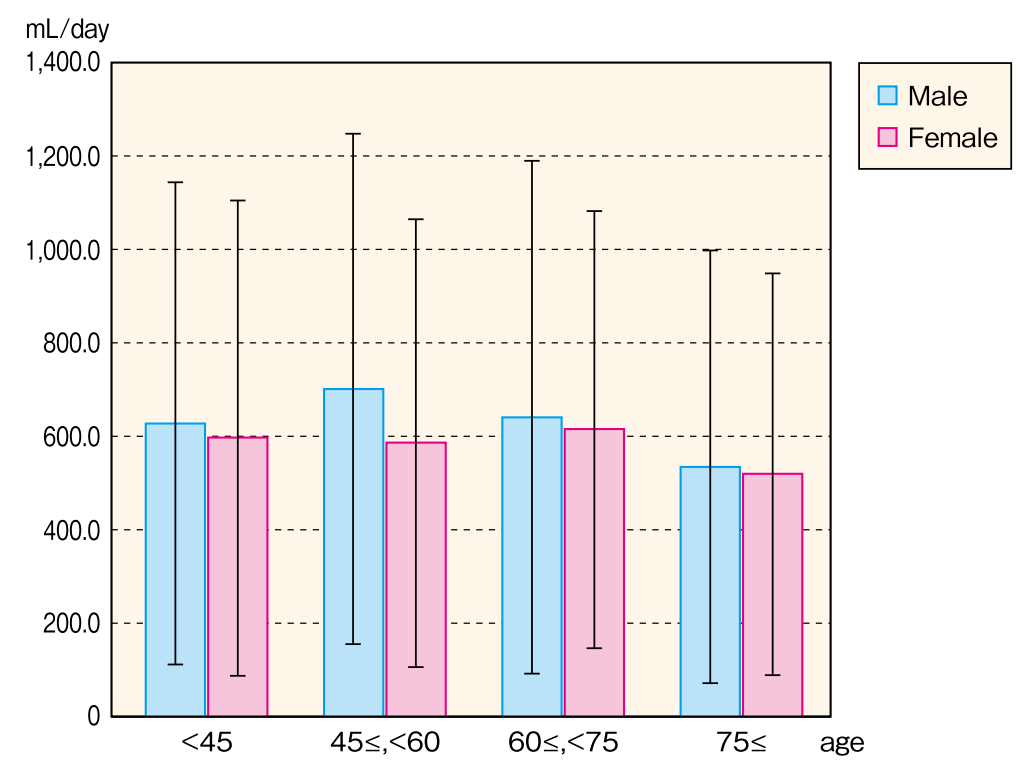

Fig. 48 Ultrafiltration volume, by age and sex, 2016

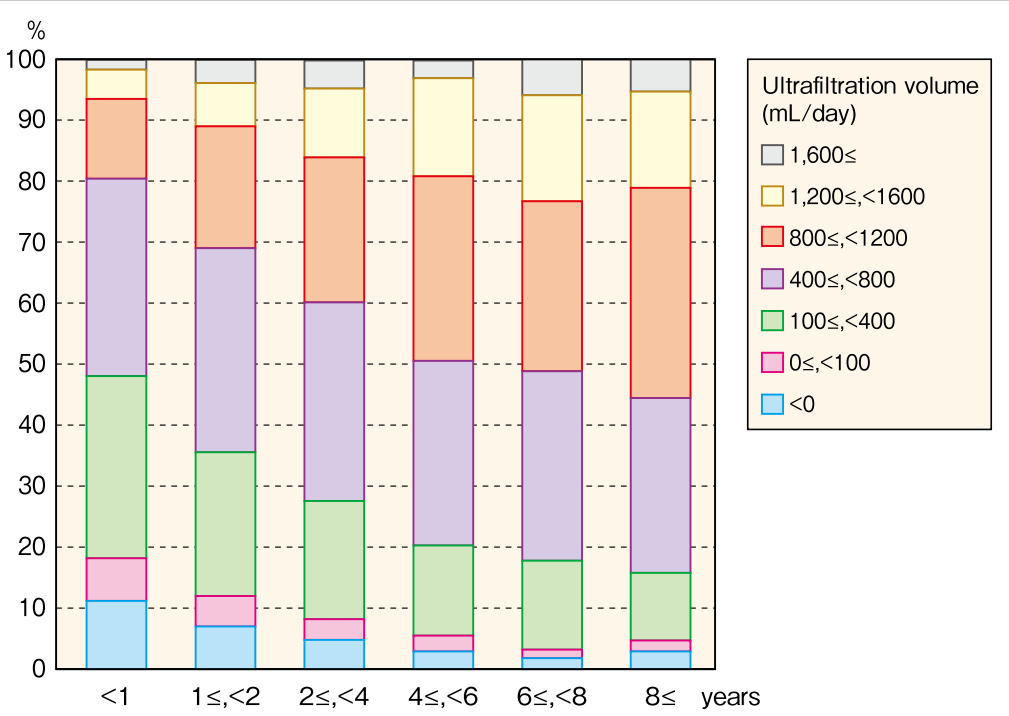

Fig. 49 Ultrafiltration volume distribution, by PD vintage, 2016. Abbreviation: PD peritoneal dialysis 


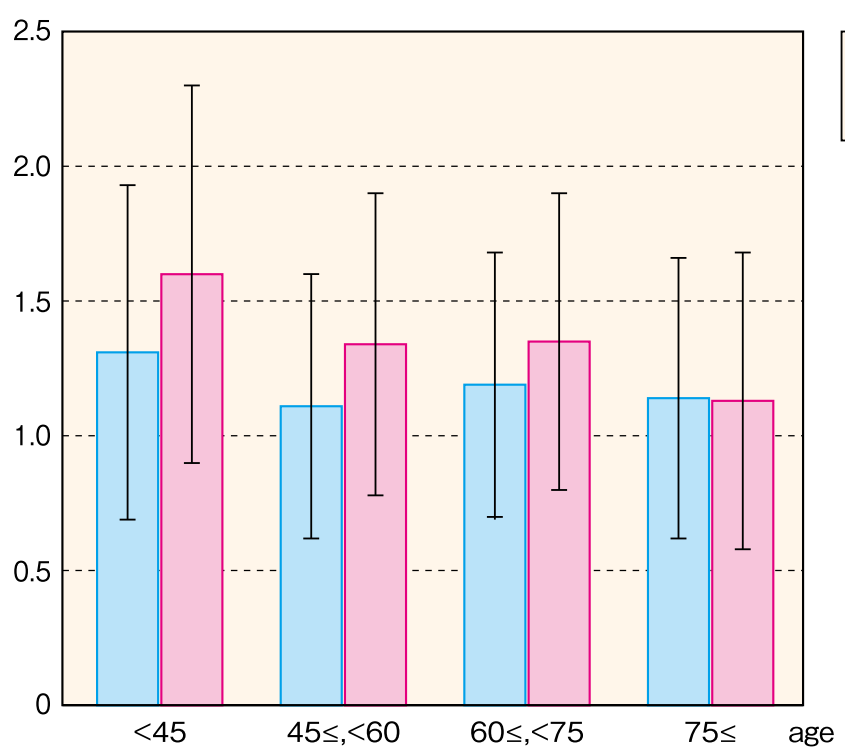

Fig. 50 PD Kt/V, by age and sex, 2016. Abbreviations: $K t / V$ index for standardized dialysis dose defined as $K$ urea clearance, $t$ dialysis time, $V$ body fluid volume; PD peritoneal dialysis

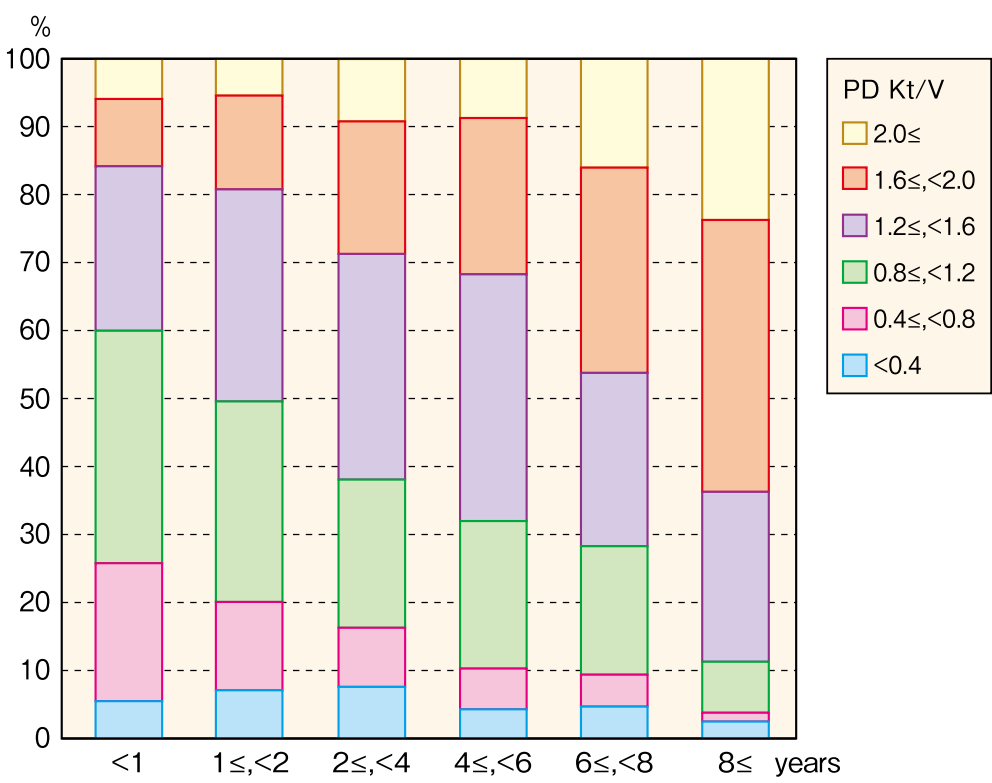

Fig. 51 PD Kt/V distribution, by PD vintage, 2016. Abbreviations: Kt/V index for standardized dialysis dose defined as $K$ urea clearance, $t$ dialysis time, $V$ body fluid volume; PD peritoneal dialysis

Table 19 History of PET, 2016

\begin{tabular}{lcccccrr}
\hline & Not performed & Standard PET & Fast PET & Subtotal & Unspecified & No information available & Total \\
\hline Patients (\%) & $1704(36.0)$ & $2173(45.9)$ & $857(18.1)$ & $4734(100.0)$ & 42 & 2155 & 6931 \\
\hline
\end{tabular}

Values in parentheses under each figure represent the percentage relative to the subtotal in each row. The above data were obtained from the patient survey. Population: PD patients excluding PD + HD combination 


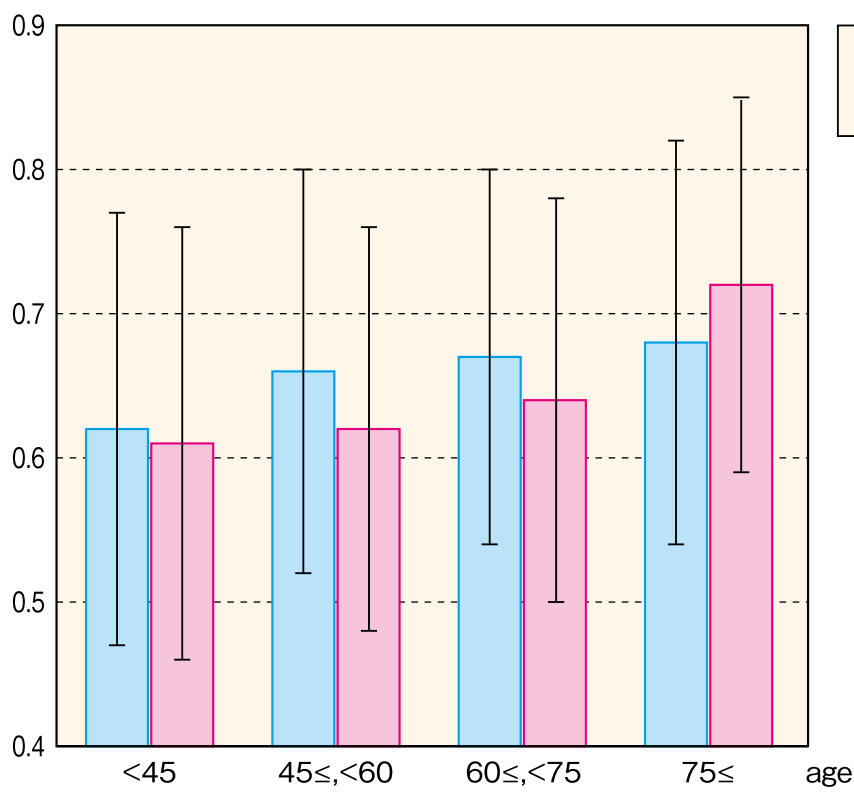

Fig. 52 D/P Cr ratio, by age and sex, 2016. Abbreviations: D/P Cr ratio dialysate/plasma creatinine ratio, PD peritoneal dialysis

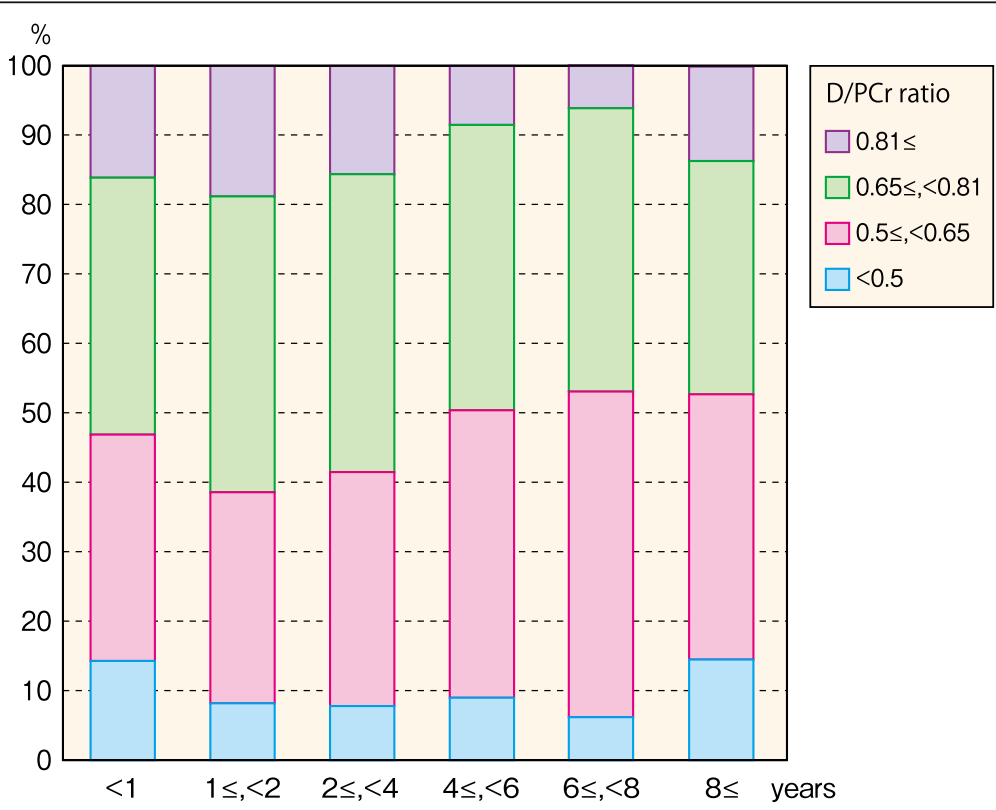

Fig. 53 D/P Cr ratio distribution, by PD vintage, 2016. Abbreviations: D/P Cr ratio dialysate/plasma creatinine ratio, PD peritoneal dialysis 


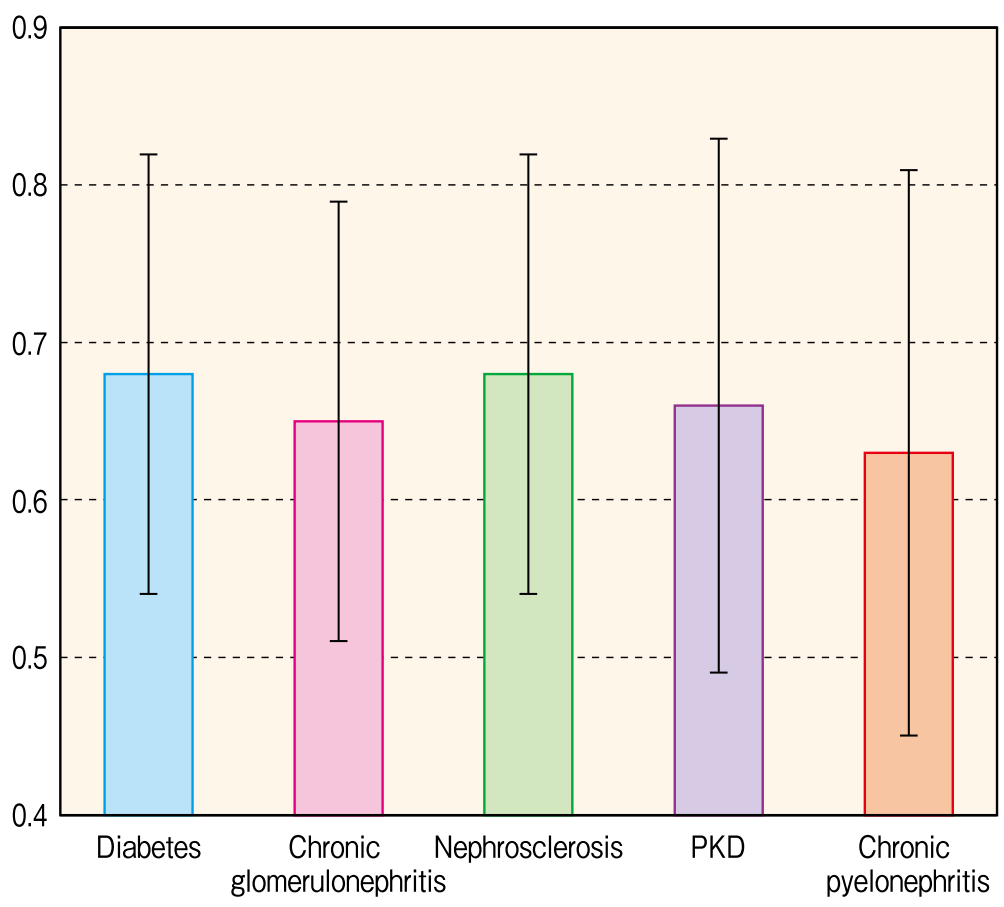

Fig. $54 \mathrm{D} / \mathrm{P} \mathrm{Cr}$ ratio, by primary disease, 2016. Abbreviations. D/P Cr ratio dialysate/plasma creatinine ratio, PD peritoneal dialysis, PKD polycystic kidney disease

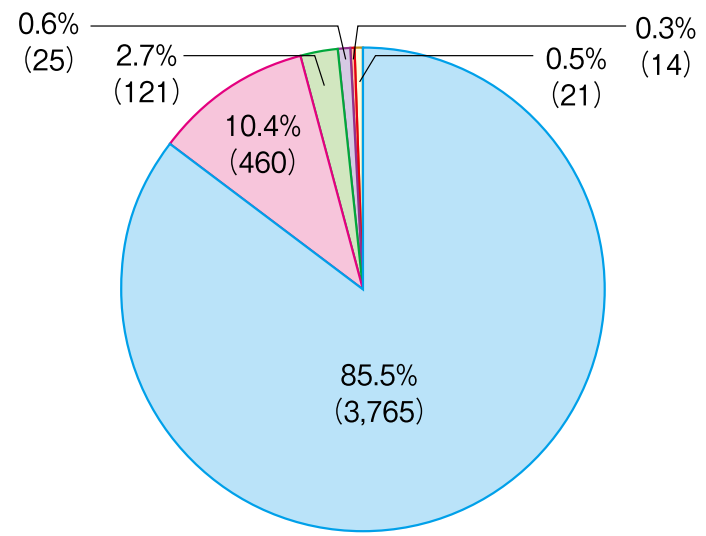

Onset rate (episodes/patient-year)

$\square 0$

$\square 1 \leq,<2$

$\square 2 \leq,<3$

$\square 3 \leq,<4$

$\square 4 \leq,<5$

$\square 5 \leq$

Fig. 55 Patient's peritonitis rate, 2016 


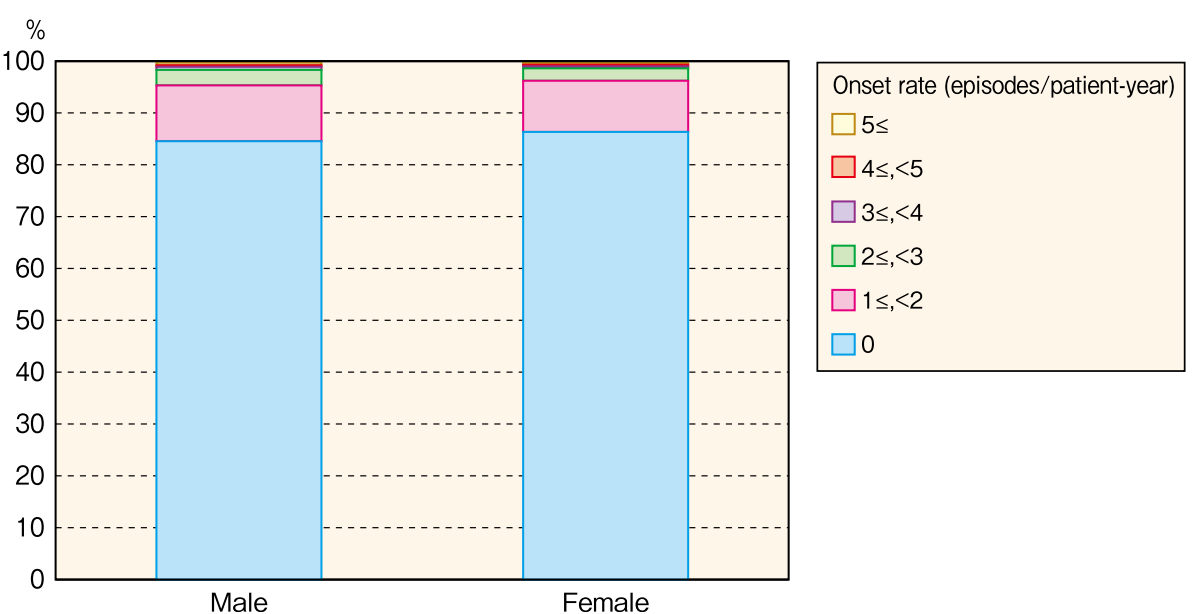

Fig. 56 Patient's peritonitis rate, by sex, 2016

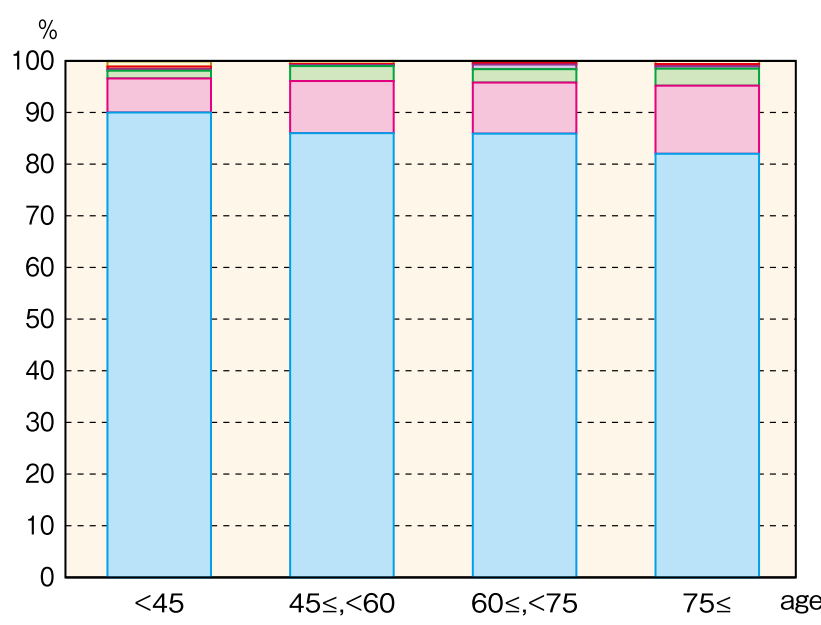

\begin{tabular}{|l|} 
Onset rate (episodes/patient-year) \\
$\square 5 \leq$ \\
$\square 4 \leq,<5$ \\
$\square 3 \leq,<4$ \\
$\square 2 \leq,<3$ \\
$\square 1 \leq,<2$ \\
$\square 0$
\end{tabular}

Fig. 57 Patient's peritonitis rate, by age, 2016

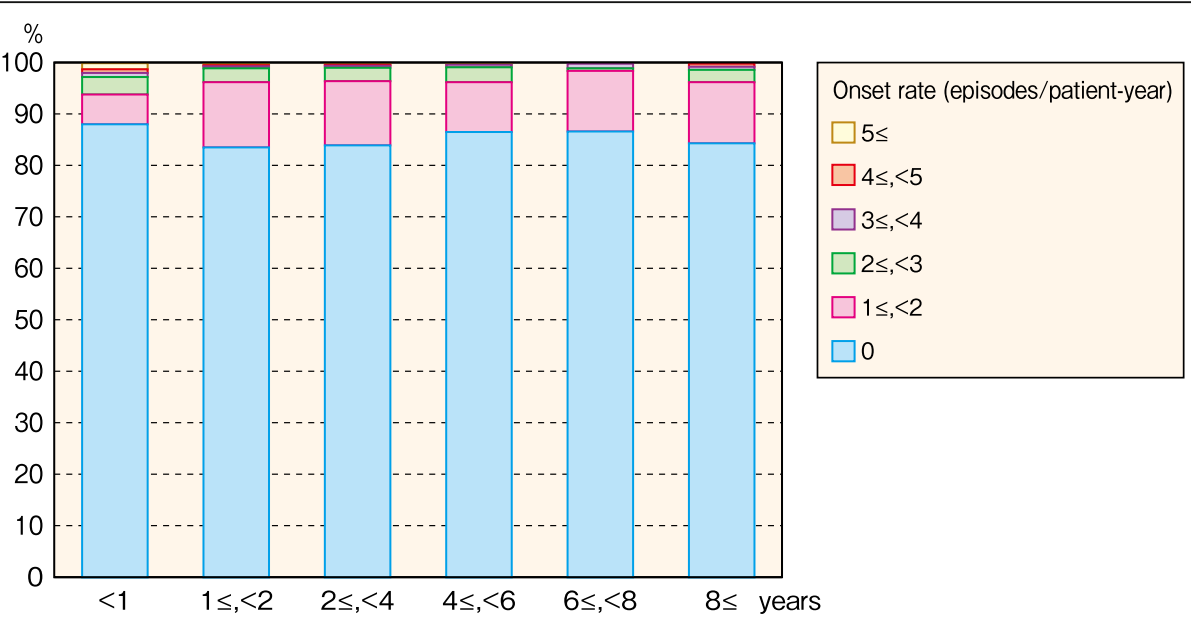

Fig. 58 Patient's peritonitis rate, by PD vintage, 2016. Abbreviation: PD peritoneal dialysis 


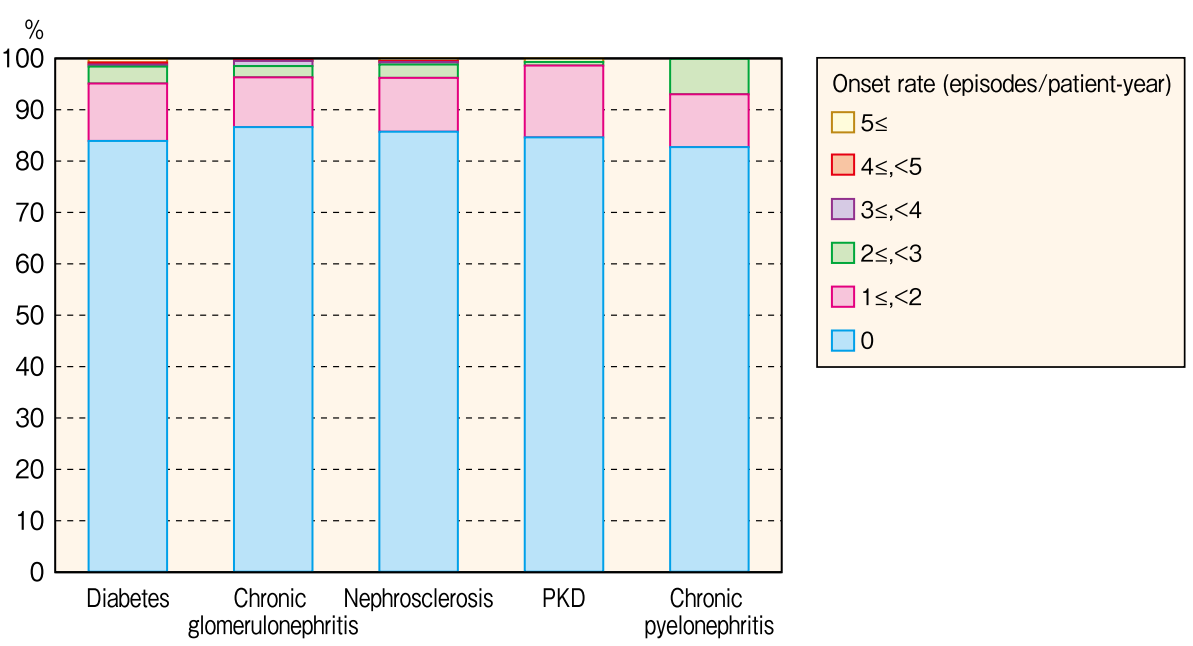

Fig. 59 Patient's peritonitis rate, by primary disease, 2016

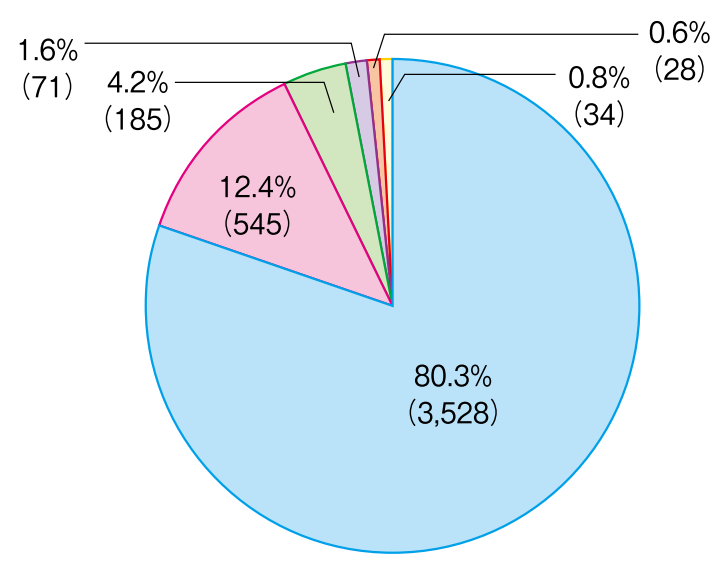

$0.6 \%$

\begin{tabular}{|l|}
\hline Onset rate (episodes/patient-year) \\
$\square 0$ \\
$\square 1 \leq,<2$ \\
$\square 2 \leq,<3$ \\
$\square 3 \leq,<4$ \\
$\square 4 \leq,<5$ \\
$\square 5 \leq$
\end{tabular}

Fig. 60 Patient's ESI rate, 2016. Abbreviation: ESI exit-site infection

Table 20 Patient with EPS history distribution, by treatment for EPS, 2016

\begin{tabular}{lcccccccc}
\hline $\begin{array}{l}\text { EPS } \\
\text { history } \\
\text { of EPS }\end{array}$ & $\begin{array}{l}\text { EPS with history } \\
\text { of surgical } \\
\text { intervention and } \\
\text { steroidal use }\end{array}$ & $\begin{array}{l}\text { EPS with history } \\
\text { of surgical } \\
\text { intervention but } \\
\text { without steroid }\end{array}$ & $\begin{array}{l}\text { EPS without } \\
\text { surgical } \\
\text { intervention but } \\
\text { with steroidal } \\
\text { use }\end{array}$ & $\begin{array}{l}\text { EPS without } \\
\text { surgical } \\
\text { intervention or } \\
\text { steroidal use }\end{array}$ & Subtotal & $\begin{array}{l}\text { Unspecified } \\
\text { No }\end{array}$ & $\begin{array}{l}\text { No } \\
\text { information } \\
\text { available }\end{array}$ \\
$\begin{array}{l}\text { Patients } \\
(\%)\end{array}$ & $12,329(94.7)$ & $502(3.9)$ & $39(0.3)$ & $80(0.6)$ & $75(0.6)$ & $13,025(100.0)$ & 265 & 3819 \\
\hline
\end{tabular}

Values in parentheses under each figure represent the percentage relative to the subtotal in each row. The above data were obtained from the patient survey. Population: PD patients excluding PD + HD combination

Abbreviations: $H D$ hemodialysis, $P D$ peritoneal dialysis, EPS encapsulating peritoneal sclerosis 


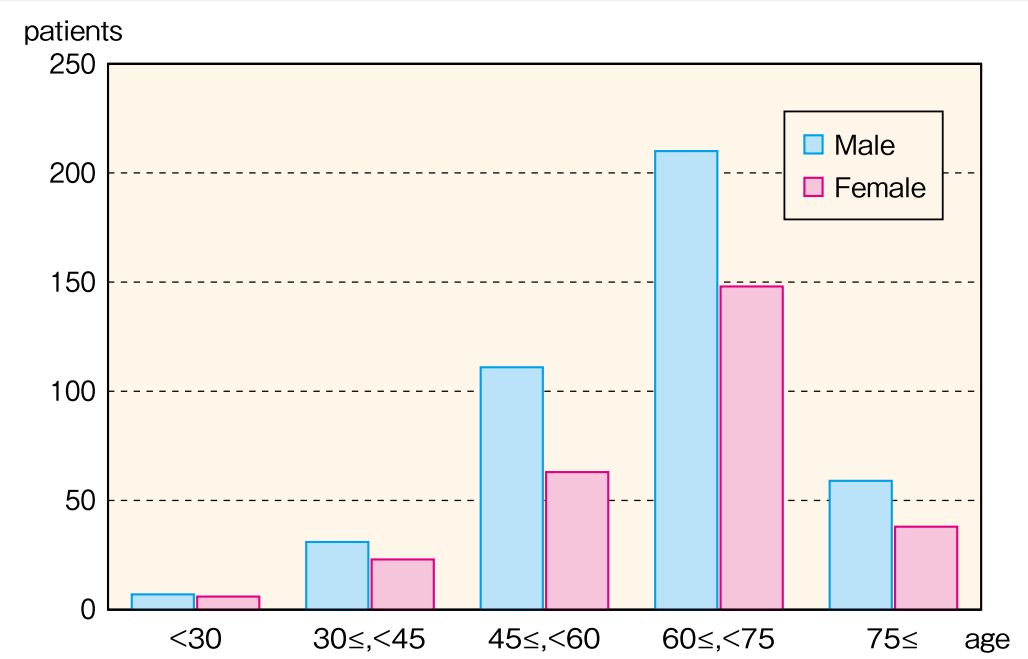

Fig. 61 Patient with EPS history distribution, by age and sex, 2016. Abbreviation: EPS encapsulating peritoneal sclerosis

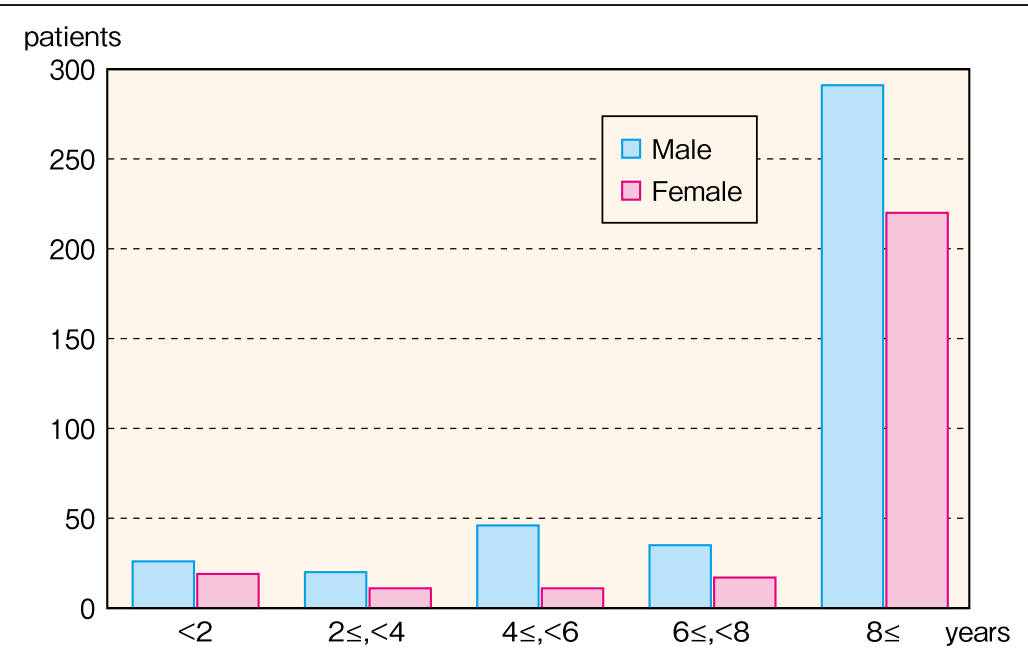

Fig. 62 Patient with EPS history distribution, by PD vintage and sex, 2016. Abbreviations: EPS encapsulating peritoneal sclerosis, PD peritoneal dialysis 
(39.9\%), and the age distribution was nearly identical to that of overall PD patients (Fig. 61, Additional file 58: Table S58). The frequency of EPS increased as the PD vintage lengthened; 511 patients with a history of EPS had a PD vintage of at least 8 years (Fig. 62, Additional file 59: Table S59).

\section{Conclusion}

To summarize the 2016 JRDR results, the number of chronic dialysis patients and dialysis facilities in Japan continued to increase, but the rate of increase has been gradually declining. The percentage of home dialysis combined with PD and HHD remained low at 3.0\%, and $\mathrm{PD}$ in particular exhibited a downward trend. Amidst calls for promoting regional comprehensive care and the necessity for home treatment as dialysis patients become older, we believe that a balanced selection of dialysis modalities is necessary. For in-center HD therapy, the number of on-line HDF patients has increased rapidly since the 2012 revision of the medical reimbursement system; HDF treatment overall now represents approximately one fourth of all in-center dialysis patients. Going forward, it will be necessary to use the JRDR data to analyze the relationships between different types of treatment variations, patient care, survival outcomes, and incidence of complications to formulate evidencebased treatment plans and revise the medical reimbursement system.

\section{Endnote}

${ }^{1}$ In the standard PET, a $2.5 \%$ dextrose solution is retained for $4 \mathrm{~h}$, and the blood $\mathrm{Cr} /$ dialysis fluid $\mathrm{Cr}$ ratio is measured at 2 and $4 \mathrm{~h}$; in fast PET, values are measured only at $4 \mathrm{~h}$.

\section{Additional files}

Additional file 1: Table S1. Changes in the number of prevalent dialysis patients, 1968-2016. (XLSX 13 kb)

Additional file 2: Table S2. Prevalent, incident, and deceased dialysis patient counts, 1983-2016. (XLSX $11 \mathrm{~kb})$

Additional file 3: Table S3. Prevalent dialysis patient distribution, by age \& sex, 2016. (XLSX $12 \mathrm{~kb}$ )

Additional file 4: Table S4. Prevalent dialysis patient distribution, by age, 1982-2016. (XLSX 13 kb)

Additional file 5: Table S5. Prevalent dialysis patient distribution, by sex \& dialysis vintage, 2016. (XLSX 12 kb)

Additional file 6: Table S6. Prevalent dialysis patient distribution, by dialysis vintage, 1988-2016. (XLSX $11 \mathrm{~kb})$

Additional file 7: Table S7. Prevalent dialysis patient distribution, by primary disease \& sex, 2016. (XLSX 14 kb)

Additional file 8: Table S8. Prevalent dialysis patient distribution, by primary disease, 1983-2016. (XLSX 16 kb)

Additional file 9: Table S9. Deceased dialysis patient distribution, by cause of death \& sex, 2016. (XLSX $12 \mathrm{~kb}$ )
Additional file 10: Table S10. Major causes of death, 1983-2016. (XLSX $12 \mathrm{~kb}$ )

Additional file 11: Table S11. Incident dialysis patient distribution, by age and sex, 2016. (XLSX $14 \mathrm{~kb}$ )

Additional file 12: Table S12. Incident dialysis patient distribution, by primary disease and sex, 2016. (XLSX 13 kb)

Additional file 13: Table S13. Incident dialysis patient distribution, by primary disease, 1983-2016. (XLSX 16 kb)

Additional file 14: Table S14. Incident patient distribution, by cause of death and sex, 2016. (XLSX $12 \mathrm{~kb})$

Additional file 15: Table S15. Causes of death during the incident year, 1990-2016. (XLSX $11 \mathrm{~kb})$

Additional file 16: Table S16. Facility distribution, by endotoxin measurement frequency \& concentration, 2016. (XLSX 12 kb)

Additional file 17: Table S17. Facility distribution, by endotoxin measurement frequency, 2006-2016. (XLSX 11 kb)

Additional file 18: Table S18. Facility distribution, by endotoxin concentration, 2006-2016. (XLSX 12 kb)

Additional file 19: Table S19. Facility distribution, by microbial measurement frequency and TVC. (XLSX $11 \mathrm{~kb}$ )

Additional file 20: Table S20. Facility distribution, by TVC measurement frequency, 2006-2016. (XLSX $11 \mathrm{~kb}$ )

Additional file 21: Table S21. Facility distribution, by TVC, 2006-2016. (XLSX $12 \mathrm{~kb}$ )

Additional file 22: Table S22. Facility distribution on TVC measurement, by cultivation medium and sampling volume, 2016. (XLSX $12 \mathrm{~kb}$ )

Additional file 23: Table S23. Facility distribution, by cultivation medium, 2006-2016. (XLSX 12 kb)

Additional file 24: Table S24. Facility distribution, by sampling volume for TVC measurement, 2006-2016. (XLSX $11 \mathrm{~kb}$ )

Additional file 25: Table S25. Facility distribution, by ETRF installation during sampling endotoxin concentration, 2016. (XLSX $11 \mathrm{~kb}$ )

Additional file 26: Table S26. Facility distribution, by ETRF installation during sampling and TVC, 2016. (XLSX $11 \mathrm{~kb}$ )

Additional file 27: Table S27. Facility distribution, by TVC and endotoxin concentration, 2016. (XLSX 12 kb)

Additional file 28: Table S28. Facility distribution, in achievement of UPD and standard dialysis fluid, 2009-2016. (XLSX $10 \mathrm{~kb}$ )

Additional file 29: Table S29. Prevalent patient distribution, by HDF modality, 2009-2016. (XLSX 12 kb)

Additional file 30: Table S30. Prevalent dialysis patient distribution, by dialysis modality and dialysis vintage, 2016. (XLSX $13 \mathrm{~kb}$ )

Additional file 31: Table S31. HDF patient distribution, by dilution mode and blood flow rate, 2016. (XLSX 12 kb)

Additional file 32: Table S32. HDF patient distribution, by dilution mode and dialysis time(minutes), 2016. (XLSX $11 \mathrm{~kb}$ )

Additional file 33: Table S33. The trend of substitutional fluid volume per session, 2012-2016. (XLSX 15 kb)

Additional file 34: Table S34. Prevalent and incident PD patient counts, 2009-2016. (XLSX 10 kb)

Additional file 35: Table S35. Prevalent patient distribution, by PD + HD combination frequency, 2009-2016. (XLSX 10 kb)

Additional file 36: Table S36. Prevalent patient distribution, by PD vintage and PD + HD combination frequency, 2016. (XLSX $11 \mathrm{~kb}$ )

Additional file 37: Table S37. PD fluid volume, by age and sex, 2016. (XLSX $9 \mathrm{~kb}$ )

Additional file 38: Table S38. Prevalent patient distribution, by PD vintage and PD fluid volume, 2016. (XLSX $12 \mathrm{~kb}$ )

Additional file 39: Table S39. PD treatment time, by age and sex, 2016. (XLSX 9 kb) 
Additional file 40: Table S40. Prevalent patient distribution, by PD vintage and PD treatment time, 2016. (XLSX $12 \mathrm{~kb}$ )

Additional file 41: Table S41. Urine volume, by age and sex, 2016. (XLSX $9 \mathrm{~kb}$ )

Additional file 42: Table S42. Prevalent PD patient distribution, by PD vintage and urine volume, 2016. (XLSX $13 \mathrm{~kb}$ )

Additional file 43: Table S43. Residual kidney $K t / V$, by age and sex, 2016. (XLSX 9 kb)

Additional file 44: Table S44. Patient distribution, PD vintage, and residual kidney $\mathrm{Kt} / \mathrm{V}$, 2016. (XLSX $13 \mathrm{~kb}$ )

Additional file 45: Table S45. Ultrafiltration volume, by age and sex, 2016. (XLSX 9 kb)

Additional file 46: Table S46. Prevalent PD patient distribution, by PD vintage and ultrafiltration volume, 2016. (XLSX 13 kb)

Additional file 47: Table S47. PD Kt/V, by age and sex, 2016. (XLSX 9 kb) Additional file 48: Table S48. Prevalent PD patient distribution, by PD vintage and PD Kt/V, 2016. (XLSX $12 \mathrm{~kb}$ )

Additional file 49: Table S49. D/P Cr ratio, by age and sex, 2016. (XLSX $9 \mathrm{~kb}$ )

Additional file 50: Table S50. PD patient distribution, by PD vintage and D/PCr ratio, 2016. (XLSX $12 \mathrm{~kb}$ )

Additional file 51: Table S51. PD patient distribution, by primary disease and D/P Cr ratio, 2016. (XLSX $10 \mathrm{~kb}$ )

Additional file 52: Table S52. Patient's peritonitis rate, 2016. (XLSX 9 kb)

Additional file 53: Table S53. Patient's peritonitis rate, by sex, 2016. (XLSX $11 \mathrm{~kb}$ )

Additional file 54: Table S54. Patient's peritonitis rate, by age, 2016. (XLSX $11 \mathrm{~kb})$

Additional file 55: Table S55. Patient's peritonitis rate, by PD vintage, 2016. (XLSX $12 \mathrm{~kb}$ )

Additional file 56: Table S56. Patient's peritonitis rate, by primary disease, 2016. (XLSX $12 \mathrm{~kb}$ )

Additional file 57: Table S57. Patient's ESI rate, 2016. (XLSX 10 kb) Additional file 58: Table S58. Patient with EPS history distribution, by age and sex, 2016. (XLSX $10 \mathrm{~kb}$ )

Additional file 59: Table S59. Patient with EPS history distribution, by dialysis vintage and sex, 2016. (XLSX $11 \mathrm{~kb}$ )

\section{Abbreviations}

\%CGR: Percent creatinine generation rate; AFBF: Acetate-free biofiltration; APD: Automated peritoneal dialysis; CD-ROM: Compact disc-read only memory; CRP: C-reactive protein; D/P Cr ratio: Dialysate/plasma creatinine ratio; EPS: Encapsulating peritoneal sclerosis; ESI: Exit-site infection; ESKD: End-stage kidney disease; ET: Endotoxin; ETRF: Endotoxin retentive filter

HD: Hemodialysis; HDF: Hemodiafiltration; HDL-C: High-density-lipoproteincholesterol concentration; HF: Hemofiltration; HHD: Home hemodialysis; IHDF: Intermittent infusion hemodiafiltration; JRDR: JSDT Renal Data Registry; JSDT: Japanese Society for Dialysis Therapy; Kt/V: Index for standardized dialysis dose defined as $K$ urea clearance, $t$ dialysis time, $V$ body fluid volume; nPCR: Normalized protein catabolic rate; PD: Peritoneal dialysis; PET: Peritoneal equilibration test; PIH: Pregnancy-induced hypertension; pmp: Per million population; PTH: Parathyroid hormone; R2A: Reasoner's no. 2 agar; RRT: Renal Replacement Therapy (an official journal of JSDT);

TAD: Therapeutic Apheresis and Dialysis (an official journal of JSDT); TGEA: Tryptone glucose extract agar; TVC: Total viable microbial count; UF: Ultrafiltration; UMIN: University hospital Medical Information Network; UMIN-CTR: UMIN Clinical Trials Registry; UN: Urea N; UPD: Ultrapure dialysis fluid; USB: Universal serial bus

\section{Acknowledgements}

We owe the completion of this survey to the efforts of the members of the subcommittee of the District Cooperative Committee members mentioned as following, and the staff members of dialysis facilities who participated in the survey and responded to the questionnaires. We would like to express our deepest gratitude to all these people.

District Cooperative Committee: Kazuyuki Maeno, Tetsuya Kawata, Chikara Oyama, Koji Seino, Toshinobu Sato, Shigeru Sato, Minoru Ito, Junichiro Kazama, Atsushi Ueda, Osamu Saito, Tetsuo Ando, Tomonari Ogawa, Hiroo Kumagai, Makoto Ogura, Takahiro Mochizuki, Ryoichi Ando, Kazuyoshi Okada, Tetsuya Kashiwagi, Chieko Hamada, Yugo Shibagaki, Nobuhito Hirawa, Hisaki Shimada, Yoichi Ishida, Hitoshi Yokoyama, Ryoichi Miyazaki, Mizuya Fukasawa, Yuji Kamijyo, Teppei Matsuoka, Akihiko Kato, Noriko Mori, Yasuhiko Ito, Hirotake Kasuga, Sukenari Koyabu, Tetsuro Arimura, Tetsuya Hashimoto, Masaaki Inaba, Terumasa Hayashi, Tomoyuki Yamakawa, Shinichi Nishi, Akira Fujimori, Tatsuo Yoneda, Shigeo Negi, Akihisa Nakaoka, Takafumi Ito, Hitoshi Sugiyama, Takao Masaki, Yutaka Nitta, Hirofumi Hashimoto, Masahito Yamanaka, Masaharu Kan, Kazumichi Ota, Masahito Tamura, Koji Mitsuiki, Yuji Ikeda, Masaharu Nishikido, Akira Miyata, Tadashi Tomo, Shoichi Fujimoto, Tsuyoshi Nosaki, and Yoshinori Oshiro.

\section{Funding}

There is no funding for the current study.

All efforts and costs for the 2015 JRDR survey and making the ADR were totally given by JSDT.

\section{Availability of data and materials}

1. When anyone wants to use the data and materials from the current manuscript without modifications, all data and materials are freely available with stating "data from JSDT."

2. When anyone wants to use the data and materials from the current manuscript with modifications, any re-calculations or something, they have to state the following sentence in their publication. "The data reported here have been provided by The Japanese Society for Dialysis Therapy (JSDT). The interpretation and reporting of these data are the responsibility of the authors and in no way should be seen as an official policy or interpretation of the JSDT."

\section{Authors' contributions}

IM was the director of CRDR in 2016 and directed all of the 2016 JRDR survey. IM and MT finalized the results of the survey and made this manuscript. SN, SO and AW designed the survey sheets and made a special program mounted in MS Excel worksheet for the convenience of selfassessment for the dialysis quality of each dialysis facility. KT, T Hase, T Hama, $\mathrm{NH}, \mathrm{JH}, \mathrm{SG}$, and $\mathrm{TH}$ had the responsibilities on the data analysis. $\mathrm{KY}$ and $\mathrm{JM}$ had the responsibility on the ethical aspect of the JRDR survey. HN was the president of JSDT in 2015 and checked all the results from the 2015 JRDR survey and approved them to be published. All authors read and approved the final manuscript.

\section{Ethics approval and consent to participate}

1. The JSDT registry was approved by the ethical committee of JSDT, the approval no. is 1 .

2. The aims of JSDT Renal Data Registry (JRDR) were well explained for the participated dialysis patients through the dialysis facilities.

3. It does not always need to get the documented approval form from the patients because all collected data were existing one and there were no new interventions.

4. The original data had been totally anonymized so there are no risks for deteriorating the privacy of the dialysis facilities and the patients.

5. The data presented in the current manuscript does not contain any images, videos, and voice recording which might have a risk for identifying an individual.

Consent for publication

Not applicable.

\section{Competing interests}

The authors declare that they have no competing interests.

\section{Publisher's Note}

Springer Nature remains neutral with regard to jurisdictional claims in published maps and institutional affiliations. 
Received: 6 August 2018 Accepted: 25 September 2018

Published online: 08 November 2018

\section{References}

1. Nakai S. The history of Japanese Society for Dialysis Therapy Registry. J Jpn Soc Dial Ther. 2010;43:119-52 (in Japanese).

2. Masakane I. Recent trends of dialysis therapy in Japan. J Jpn Soc Dial Ther 2016:49:211-8 (in Japanese).

3. Nakai S, Wakai K, Yamagata K, Iseki K, Tsubakihara Y. Prediction of dialysis patients in Japan: based on Japanese Society for Dialysis Therapy Registry. J Jpn Soc Dial Ther. 2012:45:599-613 (in Japanese).

4. Japanese society for dialysis therapy. An overview of regular dialysis treatment in Japan, the CD-ROM report (as of 31 December 2014). Tokyo: Japanese society for dialysis therapy; 2015.

5. Ethical Guidelines for Medical and Health Research Involving Human Subjects. Ministry of Health, Labor, and Welfare and Ministry of Education, Culture, Sports, Science, and Technology, Tokyo. 2017. http://www.lifescience.mext.go. jp/files/pdf/n1443_01.pdf, (last accessed 20 July 2018): in Japanese.

6. An overview of regular dialysis treatment in Japan. JSDT Renal Data Registry. Japanese Society for Dialysis Therapy, Tokyo. 2018. http://www.jsdt. or.jp/english/2426.html (last accessed 20 July 2018).

7. Masakane I, Nakai S, Ogata S, et al. Annual dialysis data report 2014, JSDT Renal Data Registry (JRDR). Ren Replace Ther. 2017;3:18. https://doi.org/10. 1186/s41100-017-0097-8.

8. Masakane I, Nakai S, Ogata S, et al. Annual dialysis data report 2015, JSDT Renal Data Registry. Ren Replace Ther. 2018;4:18. https://doi.org/10.1186/ s41100-018-0149-8

9. Chapter 11: international comparison, the 2017 USRDS Annual Data Report. Washington: United States Renal Data System, 2017. https://www.usrds.org/ 2017/view/v2_11.aspx (last accessed 20 July 2018).

10. Statistic Bureau, Home Page/Population Census, Ministry of Internal Affairs and Communications. http://www.stat.go.jp/data/jinsui/2016np/index.html (in Japanese, last access 27 July 2018).

11. Nakai $\mathrm{S}$, Iseki $\mathrm{K}$, Itami $\mathrm{N}$, et al. An overview of regular dialysis treatment in Japan (as of December 31, 2010). Ther Apher Dial. 2012;16:483-521.

12. Kawanishi H, Akiba T, Masakane I, Tomo T, Mineshima M, Kawasaki T, et al. Standard on microbiological management of fluids for hemodialysis and related therapies by The Japanese Society for Dialysis Therapy 2008. Ther Apher Dial. 2009;13:161-6.

13. Mineshima M, Kawanishi H, Ase T, Kawasaki T, Tomo T, Nakamoto H. 2016 update Japanese Society for Dialysis Therapy Standard of fluids for hemodialysis and related therapies. Ren Replace Ther. 2018;4:15. https://doi. org/10.1186/ s41100-018- 0155-x.

14. Hasegawa T, Nakai S, Masakane I, et al. Dialysis fluid endotoxin level and mortality in maintenance hemodialysis: a nationwide cohort study. Am J Kidney Dis. 2015;65:899-904.

15. Masakane I, Kawanishi H, Mineshima M, Takemoto Y, Uchono J, Hoshino T, et al. 2011 JSDT standard on the management of endotoxin retentive filter for dialysis and related therapies. Ther Apher Dial. 2013;17:229-40.

16. Masakane I, Tsubakihara Y, Akiba T, Watanabe Y, Iseki K. Bacteriological qualities of dialysis fluid in Japan as of 31 December 2006. Ther Apher Dial. 2008;12:454-160.

17. Working group committee for the preparation of guidelines for peritoneal dialysis. 2009 edition Japanese society for dialysis therapy guidelines for peritoneal dialysis. Ther Apher Dial. 2010;14:489-504.

18. Li PK, Szeto CC, Piraino B, et al. ISPD peritonitis recommendation: 2016 update on prevention and treatment. Perit Dial Int. 2016;36:481-508.

Ready to submit your research? Choose BMC and benefit from:

- fast, convenient online submission

- thorough peer review by experienced researchers in your field

- rapid publication on acceptance

- support for research data, including large and complex data types

- gold Open Access which fosters wider collaboration and increased citations

- maximum visibility for your research: over $100 \mathrm{M}$ website views per year

At $\mathrm{BMC}$, research is always in progress.

Learn more biomedcentral.com/submissions 